\title{
Chapter 3 \\ OXYGEN PRODUCTION AND DESTRUCTION
}

In this chapter, we first introduce the nuclear processes by which oxygen isotopes can be synthesised and destroyed. We then describe the main astrophysical sites where these reactions may occur, with special emphasis on massive stars and low/intermediate mass stars.

\subsection{Oxygen and nuclear physics}

Over the last decades, much experimental and theoretical work has led to the improvement of our knowledge of the rates of many thermonuclear reactions of astrophysical interest. In this section, we present an update of the $\mathrm{NACRE}^{1}$ compilation (Angulo et al. 1999) for the charged-particle induced reactions involving the stable oxygen isotopes in their entrance or exit channels, or leading in their exit channels to unstable isobars that decay to stable oxygen isotopes. The reader is referred to NACRE for the rates that do not need to be revised since the publication of this compilation. We then briefly discuss the impact of the uncertainties in these nuclear reactions on the oxygen abundance predictions. Of course, other reactions than those considered here may influence indirectly the oxygen production, but they will not be further discussed.

\subsubsection{Some generalities about thermonuclear reaction rates in astrophysical conditions}

As it is well known, the nuclear reaction rates in astrophysical plasmas are obtained by integrating the reaction cross sections $\sigma$ over a Maxwell-Boltzmann distribution of the relative energies $E$ of the reaction partners. They are classically expressed as an average of the product of $\sigma$ times the relative velocity $v$ defined as

$$
N_{A}\langle\sigma v\rangle=N_{A} \frac{(8 / \pi)^{1 / 2}}{\mu^{1 / 2}\left(k_{B} T\right)^{3 / 2}} \int \sigma(E) E \exp \left(-\frac{E}{k_{B} T}\right) d E,
$$

where $N_{A}, k_{B}, \mu$ and $T$ are the Avogadro number, Boltzmann constant, reduced mass and temperature. For charged-particle induced reactions, the astrophysical

\footnotetext{
${ }^{1}$ Nuclear Astrophysics Compilation of REaction rates. The update dates back to october 2009 . 
$S$-factor is conveniently used instead of the cross section to compensate for the rapid variation of the cross section with $E$ below the Coulomb barrier. It is defined as

$$
S(E)=E \exp (2 \pi \eta) \sigma(E)
$$

where $\eta=Z_{1} Z_{2} e^{2} / \hbar v$ is the Sommerfeld parameter, $Z_{1}$ and $Z_{2}$ being the charge numbers of the interacting nuclei.

In NACRE, Equation (3.1) is integrated numerically for the non-resonant and broad resonance contributions to the rates. The narrow resonances are treated separately. If they are approximated by Breit-Wigner forms, their contributions to the rates are functions of temperature, resonance strengths and widths. The reader is referred to Angulo et al. (1999) for details, as well as for an original analytic approximation of the rates.

A major experimental challenge in the evaluation of the rates lies in the identification of narrow resonances at the largely sub-Coulomb energies of astrophysical interest (the so-called Gamow window). Another difficulty concerns the extrapolation of the non-resonance contribution (i.e. is the $S$-factor) to these low energies, where direct experimental data are not available except in a few cases. The extrapolation techniques documented in NACRE may vary from one reaction to the other. In some cases, a simple constant value is adopted. In others, the extrapolations are based on an $R$-matrix fit or on the direct capture model. For extrapolations at the energies that are relevant to high temperatures (typically $T \gtrsim 3 \times 10^{9} \mathrm{~K}$ for the reactions considered here), Hauser-Feshbach model calculations are used.

For NACRE it has been a key goal to evaluate the rate uncertainties as a function of temperature. As a result, recommended ("adopted"), lower and upper limits of the rates are provided for the reactions under consideration, and all these rates are available in extended tabular form (whose use in stellar modelling is highly recommended) at the website http://www.astro.ulb.ac.be.

\subsubsection{Main reactions}

\subsubsection{1 ${ }^{15} \mathrm{~N}(\mathrm{p}, \gamma){ }^{16} \mathrm{O}$}

The ${ }^{15} \mathrm{~N}(\mathrm{p}, \gamma){ }^{16} \mathrm{O}$ reaction is responsible for the break-out from the cold mode of the CN cycle (labelled I in Fig. 3.1), which is in general the main energy provider in the CNO burning. As ${ }^{15} \mathrm{~N}(\mathrm{p}, \alpha){ }^{12} \mathrm{C}$ is faster than ${ }^{15} \mathrm{~N}(\mathrm{p}, \gamma){ }^{16} \mathrm{O}$ (see NACRE), this leaking from the $\mathrm{CN}$ cycle is weak, so that the ${ }^{15} \mathrm{~N}$ radiative proton captures cannot drastically affect the oxygen abundances. For compilation purposes, we however provide an update of the $\mathrm{NACRE}{ }^{15} \mathrm{~N}(\mathrm{p}, \gamma){ }^{16} \mathrm{O}$ rate. It is based on a new low-energy extrapolation of the $S$-factor proposed by Mukhamedzanov et al. (2008) who apply the asymptotic normalisation constant (ANC) method to new indirect measurements (no additional direct capture measurements have been made available since NACRE).

The ANC method relies on the assumption that the nuclear reactions at low energies are independent of the nuclear structure in the internal region, and are 


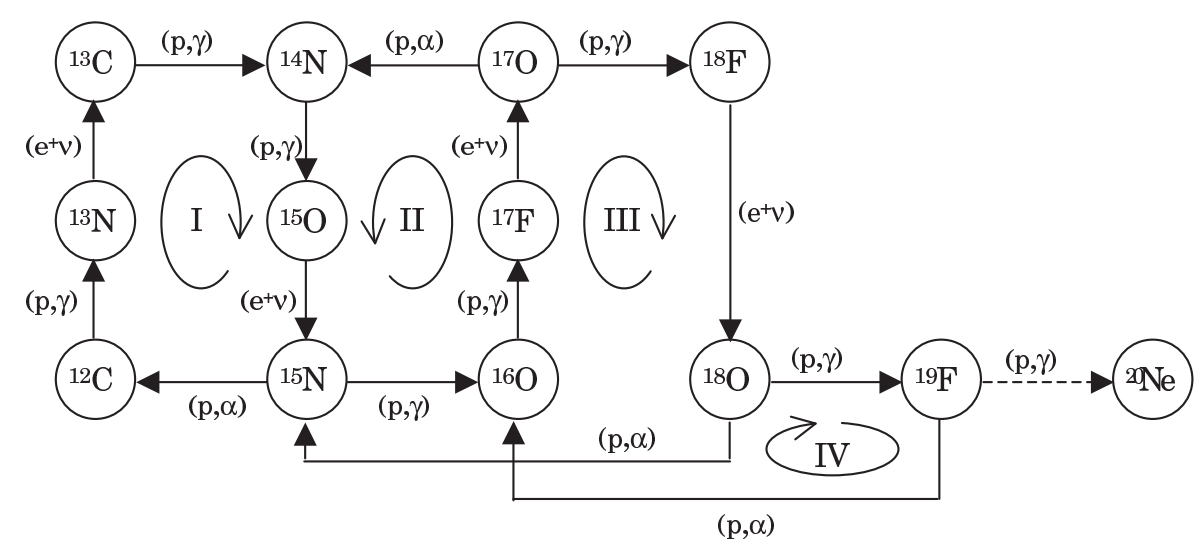

Fig. 3.1. Reactions involved in the cold CNO mode of hydrogen burning. This mode refers to situations in which the $\beta$-decay lifetimes of unstable nuclides are shorter than their lifetimes against charged particle captures. Hot modes apply in the reverse situation, and can develop mainly in explosive scenarios.

dominated instead by the wave function in the peripheral region. Mukhamedzanov et al. (2008) investigated the proton transfer reaction ${ }^{15} \mathrm{~N}\left({ }^{3} \mathrm{He}, \mathrm{d}\right){ }^{16} \mathrm{O}$, and extracted the ANCs of the ground and excited states of ${ }^{16} \mathrm{O}$ by fitting the observed angular distribution data within the DWBA approximation. The derived ANCs are proportional to the non-resonant direct capture cross section. For the resonance parameters, Mukhamedzanov et al. (2008) re-evaluated the proton and $\alpha$-particle widths by fitting the ${ }^{15} \mathrm{~N}(\mathrm{p}, \alpha){ }^{12} \mathrm{C}$ reaction rate that largely dominates ${ }^{15} \mathrm{~N}(\mathrm{p}, \gamma){ }^{16} \mathrm{O}$ at the low energies considered.

Figure 3.2 shows the $S$-factor for ${ }^{15} \mathrm{~N}(\mathrm{p}, \gamma){ }^{16} \mathrm{O}$ obtained from the combined use of the $R$-matrix and ANC methods, and with the revised proton and $\alpha$-particle widths. The total $S$-factor at zero energy is predicted to be $S(0)=36 \pm 6 \mathrm{keV}$ b, and the zero-energy $S$-factor for the non-resonant direct capture to the ground state is $0.86 \mathrm{keV} \mathrm{b}$, which is 9 times smaller than that estimated by Rolfs \& Rodney (1974). It should be noted that the ANC method relies on the reproduction of the angular distribution data derived from an indirect measurement, and thus provides only model-dependent reaction rates. We consider that the procedure adopted by Mukhamedzanov et al. (2008) is suitable for the ${ }^{15} \mathrm{~N}$ radiative capture.

The reaction rates calculated with the revised $S$-factor of Figure 3.2 are listed in Table 3.1. Below $T_{9}=0.15$, they are approximately half of the NACRE rates. As a consequence, the break-out from the CN cycle (cycle I) to the ON cycle (cycle II) is even smaller than previously expected.

\subsubsection{2 ${ }^{17} \mathrm{O}(\mathrm{p}, \alpha){ }^{14} \mathrm{~N}$ and ${ }^{17} \mathrm{O}(\mathrm{p}, \gamma){ }^{18} \mathrm{~F}$}

Figure 3.1 shows that ${ }^{17} \mathrm{O}(\mathrm{p}, \alpha)^{14} \mathrm{~N}$ closes cycle II of the CNO cycle, while a breakout to cycle III is allowed by ${ }^{17} \mathrm{O}(\mathrm{p}, \gamma){ }^{18} \mathrm{~F}$. The relative rates of these two 




Fig. 3.2. The ${ }^{15} \mathrm{~N}(\mathrm{p}, \gamma){ }^{16} \mathrm{O} S$-factor. The experimental data are from Rolfs \& Rodney (1974) and Hebbard (1960). The solid curve is obtained from the combination of the $R$-matrix and ANC methods (see text). The dashed curve is the total non-resonant capture $S$-factor obtained from the sum of the non-resonant component of eight bound states. The dotted curve is the non-resonant component for the ground state transition (from Mukhamedzanov et al. 2008).

${ }^{17} \mathrm{O}$ destruction channels are thus relevant in the calculation of the CNO yields of ${ }^{17} \mathrm{O}$ and ${ }^{18} \mathrm{O}$.

For $0.02<T_{9}<0.1$, the ${ }^{17} \mathrm{O}(\mathrm{p}, \alpha)^{14} \mathrm{~N}$ rates are dominated by the $1^{-} E_{\mathrm{r}}=$ $65.1 \mathrm{keV}$ resonance in ${ }^{18} \mathrm{~F}$ (excitation energy $E_{x}=5.6716 \mathrm{MeV}$ ). Its properties have been analysed in several experiments (see e.g. NACRE). In contrast, the characteristics of the $2^{-}$resonance at $E_{\mathrm{r}}=183.3 \mathrm{keV}$ (excitation energy $E_{x}=$ $5.7898 \mathrm{MeV}$ ) remained poorly known at the time of the NACRE compilation, compilation with large concomitant uncertainties in the rates, especially in the $0.1 \lesssim T_{9} \lesssim 0.4$ temperature range of interest in classical novae.

Recent studies of the $2^{-}$resonance have led to an improvement of the situation. Fox et al. $(2005,2004)$ derive for this resonance the strength $\omega \gamma_{p \gamma}=(1.2 \pm 0.2) \times$ $10^{-6} \mathrm{eV}$. Chafa et al. (2007) (see also Chafa et al. 2005, 2006) report the values $\omega \gamma_{p \gamma}=(2.2 \pm 0.4) \times 10^{-6} \mathrm{eV}$ and $\omega \gamma_{p \alpha}=(1.6 \pm 0.2) \times 10^{-3} \mathrm{eV}$. The latter result is confirmed by Newton et al. (2007) who obtain $\omega \gamma_{p \alpha}=(1.66 \pm 0.17) \times 10^{-3} \mathrm{eV}$.

Here we adopt the rates for ${ }^{17} \mathrm{O}(\mathrm{p}, \alpha)^{14} \mathrm{~N}$ and for ${ }^{17} \mathrm{O}(\mathrm{p}, \gamma){ }^{18}$ from Chafa et al. (2007) as the revised reaction rates. In addition to the $1^{-}$and $2^{-}$resonances reported above, these rates are calculated by taking into account the level at $E_{r}=-3.12 \mathrm{keV}\left({ }^{18} \mathrm{~F}\right.$ level at $\left.E_{x}=5.6034 \mathrm{MeV}\right)$ for which a new resonance strength is also reported by Chafa et al. (2007). The contribution from 15 levels at higher energies already considered in NACRE is also included. In addition to this resonant contribution, the direct capture process can affect the ${ }^{17} \mathrm{O}(\mathrm{p}, \gamma){ }^{18} \mathrm{~F}$ rate. 
Table 3.1. The ${ }^{15} \mathrm{~N}(\mathrm{p}, \gamma){ }^{16} \mathrm{O}$ rates calculated with the $S$-factors of Mukhamedzanov et al. (2008). The NACRE values are provided for comparison. Here and in all the following tables, the rates are in units of $\mathrm{cm}^{3} /(\mathrm{mol} \mathrm{s})$.

\begin{tabular}{lccccc}
\hline$T_{9}$ & $N_{A}\langle\sigma v\rangle$ & NACRE & $T_{9}$ & $N_{A}\langle\sigma v\rangle$ & NACRE \\
\hline 0.007 & $4.39 \mathrm{E}-25$ & $7.01 \mathrm{E}-25$ & 0.100 & $2.23 \mathrm{E}-05$ & $4.23 \mathrm{E}-05$ \\
0.008 & $1.29 \mathrm{E}-23$ & $2.08 \mathrm{E}-23$ & 0.110 & $6.10 \mathrm{E}-05$ & $1.15 \mathrm{E}-04$ \\
0.009 & $2.24 \mathrm{E}-22$ & $3.67 \mathrm{E}-22$ & 0.120 & $1.49 \mathrm{E}-04$ & $2.78 \mathrm{E}-04$ \\
0.010 & $2.62 \mathrm{E}-21$ & $4.33 \mathrm{E}-21$ & 0.130 & $3.34 \mathrm{E}-04$ & $6.16 \mathrm{E}-04$ \\
0.011 & $2.24 \mathrm{E}-20$ & $3.75 \mathrm{E}-20$ & 0.140 & $6.95 \mathrm{E}-04$ & $1.27 \mathrm{E}-03$ \\
0.012 & $1.50 \mathrm{E}-19$ & $2.54 \mathrm{E}-19$ & 0.150 & $1.36 \mathrm{E}-03$ & $2.46 \mathrm{E}-03$ \\
0.013 & $8.25 \mathrm{E}-19$ & $1.40 \mathrm{E}-18$ & 0.160 & $2.53 \mathrm{E}-03$ & $4.53 \mathrm{E}-03$ \\
0.014 & $3.83 \mathrm{E}-18$ & $6.55 \mathrm{E}-18$ & 0.180 & $7.74 \mathrm{E}-03$ & $1.35 \mathrm{E}-02$ \\
0.015 & $1.54 \mathrm{E}-17$ & $2.66 \mathrm{E}-17$ & 0.200 & $2.07 \mathrm{E}-02$ & $3.52 \mathrm{E}-02$ \\
0.016 & $5.52 \mathrm{E}-17$ & $9.56 \mathrm{E}-17$ & 0.250 & $1.55 \mathrm{E}-01$ & $2.44 \mathrm{E}-01$ \\
0.018 & $5.26 \mathrm{E}-16$ & $9.22 \mathrm{E}-16$ & 0.300 & $7.19 \mathrm{E}-01$ & $1.05 \mathrm{E}+00$ \\
0.020 & $3.67 \mathrm{E}-15$ & $6.50 \mathrm{E}-15$ & 0.350 & $2.33 \mathrm{E}+00$ & $3.17 \mathrm{E}+00$ \\
0.025 & $1.80 \mathrm{E}-13$ & $3.25 \mathrm{E}-13$ & 0.400 & $5.80 \mathrm{E}+00$ & $7.48 \mathrm{E}+00$ \\
0.030 & $3.51 \mathrm{E}-12$ & $6.41 \mathrm{E}-12$ & 0.450 & $1.20 \mathrm{E}+01$ & $1.49 \mathrm{E}+01$ \\
0.040 & $2.66 \mathrm{E}-10$ & $4.96 \mathrm{E}-10$ & 0.500 & $2.14 \mathrm{E}+01$ & $2.58 \mathrm{E}+01$ \\
0.050 & $5.78 \mathrm{E}-09$ & $1.09 \mathrm{E}-08$ & 0.600 & $5.11 \mathrm{E}+01$ & $5.90 \mathrm{E}+01$ \\
0.060 & $6.08 \mathrm{E}-08$ & $1.16 \mathrm{E}-07$ & 0.700 & $9.50 \mathrm{E}+01$ & $1.07 \mathrm{E}+02$ \\
0.070 & $4.00 \mathrm{E}-07$ & $7.64 \mathrm{E}-07$ & 0.800 & $1.53 \mathrm{E}+02$ & $1.68 \mathrm{E}+02$ \\
0.080 & $1.90 \mathrm{E}-06$ & $3.63 \mathrm{E}-06$ & 0.900 & $2.27 \mathrm{E}+02$ & $2.46 \mathrm{E}+02$ \\
0.090 & $7.11 \mathrm{E}-06$ & $1.36 \mathrm{E}-05$ & 1.000 & $3.25 \mathrm{E}+02$ & $3.50 \mathrm{E}+02$ \\
\hline
\end{tabular}

The low-energy $S$-factor as revised by Chafa et al. $(2007)$ is given by $S_{D C}(E)=$ $6.2+1.61 E-0.169 E^{2} \mathrm{keV}$ b, where $E$ is in $\mathrm{MeV}$.

The new reaction rates are listed in Tables 3.2 and 3.3. The errors are estimated from a Monte Carlo analysis. The ratio of the new ${ }^{17} \mathrm{O}(\mathrm{p}, \alpha)^{14} \mathrm{~N}$ rates to those in NACRE is shown in Figure 3.3. The difference for $T_{9}<0.02$ is mainly due to the lower value of the single-particle width of the subthreshold level at $E_{x}=$ $5.603 \mathrm{MeV}$. For $0.1<T_{9}<0.4$, the reaction rates increase because of the new data for the $183.3 \mathrm{keV}$ resonance. With the $S_{D C}$-factor of Chafa et al. (2007), the direct capture process affects the rates for $T_{9}<0.8$.

For ${ }^{17} \mathrm{O}(\mathrm{p}, \gamma){ }^{18} \mathrm{~F}$, the revised rates are in agreement with NACRE above $T_{9}=$ 0.4, as shown in Figure 3.4. For $0.1<T_{9}<0.4$, the new rates are smaller than the NACRE values by up to a factor of about 5 . This deviation comes from the new data for the $183.3 \mathrm{keV}$ resonance.

\subsubsection{3 ${ }^{18} \mathrm{O}(\mathrm{p}, \alpha){ }^{15} \mathrm{~N}$}

According to the NACRE rates, the burning of ${ }^{18} \mathrm{O}$ predominantly proceeds through ${ }^{18} \mathrm{O}(\mathrm{p}, \alpha){ }^{15} \mathrm{~N}$ in the $T_{9} \lesssim 0.02$ and $T_{9} \gtrsim 0.7$ regions, leading to the closure of cycle III as shown in Figure 3.1. However, for $0.02 \lesssim T_{9} \lesssim 0.7,{ }^{18} \mathrm{O}(\mathrm{p}, \gamma){ }^{19} \mathrm{~F}$ is responsible for a leak out of cycle III. 
Table 3.2. The ${ }^{17} \mathrm{O}(\mathrm{p}, \alpha){ }^{14} \mathrm{~N}$ rates from Chafa et al. (2007).

\begin{tabular}{lccclccc}
\hline$T_{9}$ & Lower & Rate & Upper & $T_{9}$ & Lower & Rate & Upper \\
\hline 0.01 & $4.02 \mathrm{E}-25$ & $4.97 \mathrm{E}-25$ & $5.92 \mathrm{E}-25$ & 0.13 & $4.35 \mathrm{E}-4$ & $4.96 \mathrm{E}-4$ & $5.57 \mathrm{E}-4$ \\
0.011 & $4.27 \mathrm{E}-24$ & $5.23 \mathrm{E}-24$ & $6.18 \mathrm{E}-24$ & 0.14 & $1.18 \mathrm{E}-3$ & $1.35 \mathrm{E}-3$ & $1.52 \mathrm{E}-3$ \\
0.012 & $3.50 \mathrm{E}-23$ & $4.24 \mathrm{E}-23$ & $4.99 \mathrm{E}-23$ & 0.15 & $2.85 \mathrm{E}-3$ & $3.27 \mathrm{E}-3$ & $3.69 \mathrm{E}-3$ \\
0.013 & $2.31 \mathrm{E}-22$ & $2.79 \mathrm{E}-22$ & $3.26 \mathrm{E}-22$ & 0.16 & $6.19 \mathrm{E}-3$ & $7.12 \mathrm{E}-3$ & $8.04 \mathrm{E}-3$ \\
0.014 & $1.29 \mathrm{E}-21$ & $1.54 \mathrm{E}-21$ & $1.80 \mathrm{E}-21$ & 0.18 & $2.25 \mathrm{E}-2$ & $2.59 \mathrm{E}-2$ & $2.92 \mathrm{E}-2$ \\
0.015 & $6.23 \mathrm{E}-21$ & $7.44 \mathrm{E}-21$ & $8.65 \mathrm{E}-21$ & 0.2 & $6.25 \mathrm{E}-2$ & $7.18 \mathrm{E}-2$ & $8.11 \mathrm{E}-2$ \\
0.016 & $2.75 \mathrm{E}-20$ & $3.26 \mathrm{E}-20$ & $3.77 \mathrm{E}-20$ & 0.25 & $3.84 \mathrm{E}-1$ & $4.39 \mathrm{E}-1$ & $4.94 \mathrm{E}-1$ \\
0.018 & $5.12 \mathrm{E}-19$ & $6.19 \mathrm{E}-19$ & $7.26 \mathrm{E}-19$ & 0.3 & $1.48 \mathrm{E}+0$ & $1.65 \mathrm{E}+0$ & $1.82 \mathrm{E}+0$ \\
0.02 & $1.11 \mathrm{E}-17$ & $1.53 \mathrm{E}-17$ & $1.94 \mathrm{E}-17$ & 0.35 & $6.02 \mathrm{E}+0$ & $6.50 \mathrm{E}+0$ & $6.97 \mathrm{E}+0$ \\
0.025 & $1.10 \mathrm{E}-14$ & $1.54 \mathrm{E}-14$ & $1.99 \mathrm{E}-14$ & 0.4 & $2.56 \mathrm{E}+1$ & $2.75 \mathrm{E}+1$ & $2.94 \mathrm{E}+1$ \\
0.03 & $1.30 \mathrm{E}-12$ & $1.76 \mathrm{E}-12$ & $2.22 \mathrm{E}-12$ & 0.45 & $9.28 \mathrm{E}+1$ & $9.99 \mathrm{E}+1$ & $1.07 \mathrm{E}+2$ \\
0.04 & $4.77 \mathrm{E}-10$ & $6.14 \mathrm{E}-10$ & $7.52 \mathrm{E}-10$ & 0.5 & $2.73 \mathrm{E}+2$ & $2.95 \mathrm{E}+2$ & $3.16 \mathrm{E}+2$ \\
0.05 & $1.52 \mathrm{E}-8$ & $1.92 \mathrm{E}-8$ & $2.32 \mathrm{E}-8$ & 0.6 & $1.42 \mathrm{E}+3$ & $1.53 \mathrm{E}+3$ & $1.63 \mathrm{E}+3$ \\
0.06 & $1.46 \mathrm{E}-7$ & $1.81 \mathrm{E}-7$ & $2.17 \mathrm{E}-7$ & 0.7 & $4.64 \mathrm{E}+3$ & $4.96 \mathrm{E}+3$ & $5.28 \mathrm{E}+3$ \\
0.07 & $7.09 \mathrm{E}-7$ & $8.73 \mathrm{E}-7$ & $1.04 \mathrm{E}-6$ & 0.8 & $1.12 \mathrm{E}+4$ & $1.20 \mathrm{E}+4$ & $1.27 \mathrm{E}+4$ \\
0.08 & $2.28 \mathrm{E}-6$ & $2.79 \mathrm{E}-6$ & $3.30 \mathrm{E}-6$ & 0.9 & $2.24 \mathrm{E}+4$ & $2.38 \mathrm{E}+4$ & $2.51 \mathrm{E}+4$ \\
0.09 & $5.96 \mathrm{E}-6$ & $7.16 \mathrm{E}-6$ & $8.36 \mathrm{E}-6$ & 1.0 & $3.91 \mathrm{E}+4$ & $4.12 \mathrm{E}+4$ & $4.34 \mathrm{E}+4$ \\
0.1 & $1.56 \mathrm{E}-5$ & $1.80 \mathrm{E}-5$ & $2.05 \mathrm{E}-5$ & 1.25 & $1.09 \mathrm{E}+5$ & $1.14 \mathrm{E}+5$ & $1.19 \mathrm{E}+5$ \\
0.11 & $4.62 \mathrm{E}-5$ & $5.20 \mathrm{E}-5$ & $5.77 \mathrm{E}-5$ & 1.5 & $2.29 \mathrm{E}+5$ & $2.38 \mathrm{E}+5$ & $2.47 \mathrm{E}+5$ \\
0.12 & $1.45 \mathrm{E}-4$ & $1.64 \mathrm{E}-4$ & $1.83 \mathrm{E}-4$ & & & & \\
\hline
\end{tabular}

Table 3.3. The ${ }^{17} \mathrm{O}(\mathrm{p}, \gamma){ }^{18} \mathrm{~F}$ rates from Chafa et al. (2007).

\begin{tabular}{lccclccc}
\hline$T_{9}$ & Lower & Rate & Upper & $T_{9}$ & Lower & Rate & Upper \\
\hline 0.01 & $2.37 \mathrm{E}-25$ & $4.55 \mathrm{E}-25$ & $6.73 \mathrm{E}-25$ & 0.13 & $1.84 \mathrm{E}-6$ & $2.79 \mathrm{E}-6$ & $3.74 \mathrm{E}-6$ \\
0.011 & $2.52 \mathrm{E}-24$ & $4.84 \mathrm{E}-24$ & $7.15 \mathrm{E}-24$ & 0.14 & $4.25 \mathrm{E}-6$ & $6.29 \mathrm{E}-6$ & $8.33 \mathrm{E}-6$ \\
0.012 & $2.04 \mathrm{E}-23$ & $3.91 \mathrm{E}-23$ & $5.79 \mathrm{E}-23$ & 0.15 & $9.16 \mathrm{E}-6$ & $1.32 \mathrm{E}-5$ & $1.73 \mathrm{E}-5$ \\
0.013 & $1.32 \mathrm{E}-22$ & $2.54 \mathrm{E}-22$ & $3.76 \mathrm{E}-22$ & 0.16 & $1.84 \mathrm{E}-5$ & $2.61 \mathrm{E}-5$ & $3.38 \mathrm{E}-5$ \\
0.014 & $7.14 \mathrm{E}-22$ & $1.37 \mathrm{E}-21$ & $2.03 \mathrm{E}-21$ & 0.18 & $6.13 \mathrm{E}-5$ & $8.50 \mathrm{E}-5$ & $1.09 \mathrm{E}-4$ \\
0.015 & $3.30 \mathrm{E}-21$ & $6.34 \mathrm{E}-21$ & $9.37 \mathrm{E}-21$ & 0.2 & $1.66 \mathrm{E}-4$ & $2.27 \mathrm{E}-4$ & $2.89 \mathrm{E}-4$ \\
0.016 & $1.34 \mathrm{E}-20$ & $2.57 \mathrm{E}-20$ & $3.80 \mathrm{E}-20$ & 0.25 & $1.07 \mathrm{E}-3$ & $1.48 \mathrm{E}-3$ & $1.89 \mathrm{E}-3$ \\
0.018 & $1.60 \mathrm{E}-19$ & $3.07 \mathrm{E}-19$ & $4.53 \mathrm{E}-19$ & 0.3 & $4.24 \mathrm{E}-3$ & $5.98 \mathrm{E}-3$ & $7.71 \mathrm{E}-3$ \\
0.02 & $1.39 \mathrm{E}-18$ & $2.62 \mathrm{E}-18$ & $3.85 \mathrm{E}-18$ & 0.35 & $1.53 \mathrm{E}-2$ & $2.08 \mathrm{E}-2$ & $2.62 \mathrm{E}-2$ \\
0.025 & $1.47 \mathrm{E}-16$ & $2.37 \mathrm{E}-16$ & $3.26 \mathrm{E}-16$ & 0.4 & $5.73 \mathrm{E}-2$ & $7.20 \mathrm{E}-2$ & $8.67 \mathrm{E}-2$ \\
0.03 & $8.02 \mathrm{E}-15$ & $1.08 \mathrm{E}-14$ & $1.36 \mathrm{E}-14$ & 0.45 & $2.01 \mathrm{E}-1$ & $2.38 \mathrm{E}-1$ & $2.75 \mathrm{E}-1$ \\
0.04 & $2.10 \mathrm{E}-12$ & $2.66 \mathrm{E}-12$ & $3.23 \mathrm{E}-12$ & 0.5 & $6.02 \mathrm{E}-1$ & $6.95 \mathrm{E}-1$ & $7.89 \mathrm{E}-1$ \\
0.05 & $6.55 \mathrm{E}-11$ & $8.19 \mathrm{E}-11$ & $9.83 \mathrm{E}-11$ & 0.6 & $3.41 \mathrm{E}+0$ & $3.88 \mathrm{E}+0$ & $4.35 \mathrm{E}+0$ \\
0.06 & $6.66 \mathrm{E}-10$ & $8.27 \mathrm{E}-10$ & $9.89 \mathrm{E}-10$ & 0.7 & $1.21 \mathrm{E}+1$ & $1.37 \mathrm{E}+1$ & $1.53 \mathrm{E}+1$ \\
0.07 & $3.60 \mathrm{E}-9$ & $4.55 \mathrm{E}-9$ & $5.50 \mathrm{E}-9$ & 0.8 & $3.14 \mathrm{E}+1$ & $3.55 \mathrm{E}+1$ & $3.95 \mathrm{E}+1$ \\
0.08 & $1.35 \mathrm{E}-8$ & $1.78 \mathrm{E}-8$ & $2.22 \mathrm{E}-8$ & 0.9 & $6.57 \mathrm{E}+1$ & $7.38 \mathrm{E}+1$ & $8.19 \mathrm{E}+1$ \\
0.09 & $4.09 \mathrm{E}-8$ & $5.77 \mathrm{E}-8$ & $7.44 \mathrm{E}-8$ & 1.0 & $1.18 \mathrm{E}+2$ & $1.32 \mathrm{E}+2$ & $1.45 \mathrm{E}+2$ \\
0.1 & $1.12 \mathrm{E}-7$ & $1.68 \mathrm{E}-7$ & $2.23 \mathrm{E}-7$ & 1.25 & $3.26 \mathrm{E}+2$ & $3.64 \mathrm{E}+2$ & $4.03 \mathrm{E}+2$ \\
0.11 & $2.95 \mathrm{E}-7$ & $4.54 \mathrm{E}-7$ & $6.13 \mathrm{E}-7$ & 1.5 & $6.34 \mathrm{E}+2$ & $6.98 \mathrm{E}+2$ & $7.62 \mathrm{E}+2$ \\
0.12 & $7.53 \mathrm{E}-7$ & $1.16 \mathrm{E}-6$ & $1.57 \mathrm{E}-6$ & & & & \\
\hline
\end{tabular}




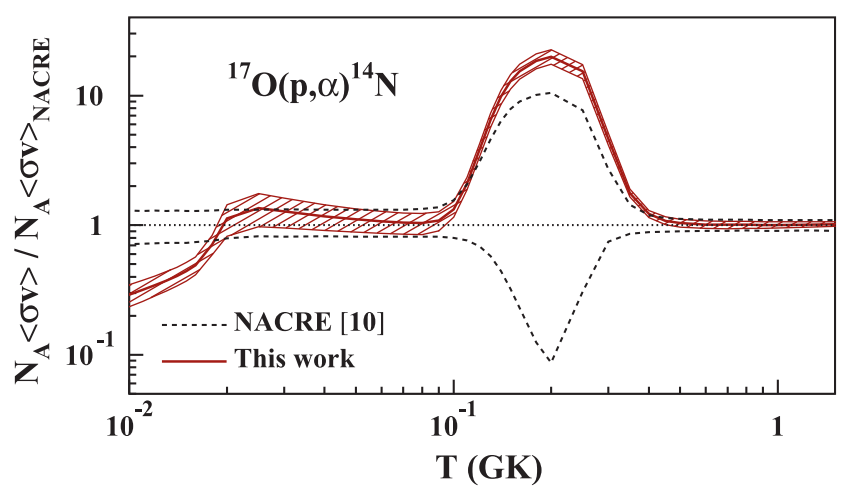

Fig. 3.3. Ratio of the Chafa et al. (2007) (labelled "This work") and NACRE rates for ${ }^{17} \mathrm{O}(\mathrm{p}, \alpha){ }^{14} \mathrm{~N}$. The hatched area represents the uncertainties in the reaction rates. $T(\mathrm{GK})$ is the temperature in $10^{9} \mathrm{~K}$ (generally expressed as $T_{9}$ ). Figure adopted from Chafa et al. (2007).
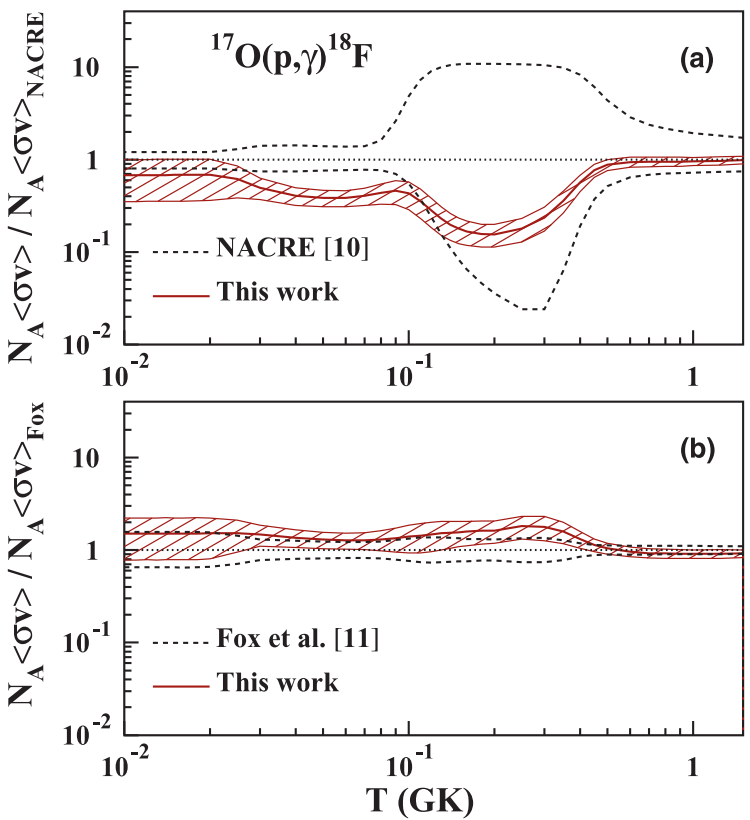

Fig. 3.4. Ratio of the Chafa et al. (2007) (labelled "This work") to (a) the NACRE rates, and (b) the rates of Fox et al. (2005) for ${ }^{17} \mathrm{O}(\mathrm{p}, \gamma){ }^{18} \mathrm{~F}$. The hatched areas represent the uncertainties in the reaction rates. Figure adopted from Chafa et al. (2007).

The narrow resonances at 20 and $143.5 \mathrm{keV}$ and the broad one at $656 \mathrm{keV}$ in ${ }^{19} \mathrm{~F}$ dominate the ${ }^{18} \mathrm{O}(\mathrm{p}, \alpha){ }^{15} \mathrm{~N}$ NACRE rates. In fact the $656 \mathrm{keV}$ resonance gives 
Table 3.4. Revised ${ }^{18} \mathrm{O}(\mathrm{p}, \alpha){ }^{15} \mathrm{~N}$ rates based on the experiments of La Cognata et al. (2008) (referred to as LA08).

\begin{tabular}{lcclcc}
\hline$T_{9}$ & LA08 & NACRE & $T_{9}$ & LA08 & NACRE \\
\hline 0.007 & $1.09 \mathrm{E}-24$ & $8.32 \mathrm{E}-25$ & 0.018 & $1.75 \mathrm{E}-15$ & $1.71 \mathrm{E}-15$ \\
0.008 & $5.48 \mathrm{E}-23$ & $4.13 \mathrm{E}-23$ & 0.02 & $1.41 \mathrm{E}-14$ & $1.40 \mathrm{E}-14$ \\
0.009 & $1.17 \mathrm{E}-21$ & $8.85 \mathrm{E}-22$ & 0.025 & $9.95 \mathrm{E}-13$ & $9.94 \mathrm{E}-13$ \\
0.01 & $1.37 \mathrm{E}-20$ & $1.05 \mathrm{E}-20$ & 0.03 & $2.61 \mathrm{E}-11$ & $2.61 \mathrm{E}-11$ \\
0.011 & $1.06 \mathrm{E}-19$ & $8.34 \mathrm{E}-20$ & 0.04 & $3.07 \mathrm{E}-09$ & $3.07 \mathrm{E}-09$ \\
0.012 & $6.16 \mathrm{E}-19$ & $5.00 \mathrm{E}-19$ & 0.05 & $1.01 \mathrm{E}-07$ & $1.01 \mathrm{E}-07$ \\
0.013 & $2.94 \mathrm{E}-18$ & $2.48 \mathrm{E}-18$ & 0.06 & $2.92 \mathrm{E}-06$ & $2.91 \mathrm{E}-06$ \\
0.014 & $1.23 \mathrm{E}-17$ & $1.08 \mathrm{E}-17$ & 0.07 & $7.94 \mathrm{E}-05$ & $7.93 \mathrm{E}-05$ \\
0.015 & $4.71 \mathrm{E}-17$ & $4.31 \mathrm{E}-17$ & 0.08 & $1.17 \mathrm{E}-03$ & $1.17 \mathrm{E}-03$ \\
0.016 & $1.68 \mathrm{E}-16$ & $1.58 \mathrm{E}-16$ & 0.09 & $9.63 \mathrm{E}-03$ & $9.63 \mathrm{E}-03$ \\
\hline
\end{tabular}

a strong contribution in the entire temperature range considered in NACRE. The $143.5 \mathrm{keV}$ resonance was reported by NACRE to be fairly well known. In contrast, uncertainties remained in the $20 \mathrm{keV}$ resonance properties, with concomitant uncertainties in the rate. This was mainly due to the adoption of the resonance strength predicted by the DWBA analysis of the proton transfer reactions and from direct capture measurements. In addition, a resonance at $90 \mathrm{keV}$ had an unknown $J^{\pi}$ value, so that its contribution to the rate could not be ascertained.

To reduce the uncertainties in the reaction rates, the 20 and $90 \mathrm{keV}$ resonances have been investigated by means of the Trojan horse method (La Cognata et al. 2008), which is an indirect technique to evaluate cross sections at energies well below the Coulomb barrier. The strengths of the 20 and $90 \mathrm{keV}$ resonances derived in such a way are $\omega \gamma=8.3_{-2.6}^{+3.8} \times 10^{-19} \mathrm{eV}$ and $(1.76 \pm 0.33) \times 10^{-7} \mathrm{eV}$, respectively. The rates provided in Table 3.4 are obtained by updating the NACRE predictions with these new resonance strengths. This leads to a marginal increase of the original NACRE rates, but also to a significant reduction of the uncertainties around $T_{9}=0.01$, as shown in Figure 3.5.

\subsubsection{4 ${ }^{12} \mathrm{C}(\alpha, \gamma){ }^{16} \mathrm{O}$}

This reaction is classically considered as one of the most important in stellar physics. It influences the structure and nucleosynthesis of the stars during their central He-burning phase, as well as during the subsequent nuclear burning stages in massive stars up to their final (supernova) fate. No wonder then that the radiative capture of $\alpha$-particles by ${ }^{12} \mathrm{C}$ at energies of astrophysical relevance (around a centre-of-mass energy of about $300 \mathrm{keV}$ for hydrostatic He-burning conditions) has been one of the most studied reactions. Both direct and indirect techniques have been applied to measure the cross sections or the quantities entering their estimate. In recent years, precise measurements of $\gamma$-ray angular distributions and of cascade transitions have also accumulated (Assunção et al. 2006; Hammer et al. 2005; Kunz et al. 2001; Matei et al. 2006; Roters et al. 1999; Tischhauser et al. 2002). In spite of all these efforts, uncertainties that are generally considered to 


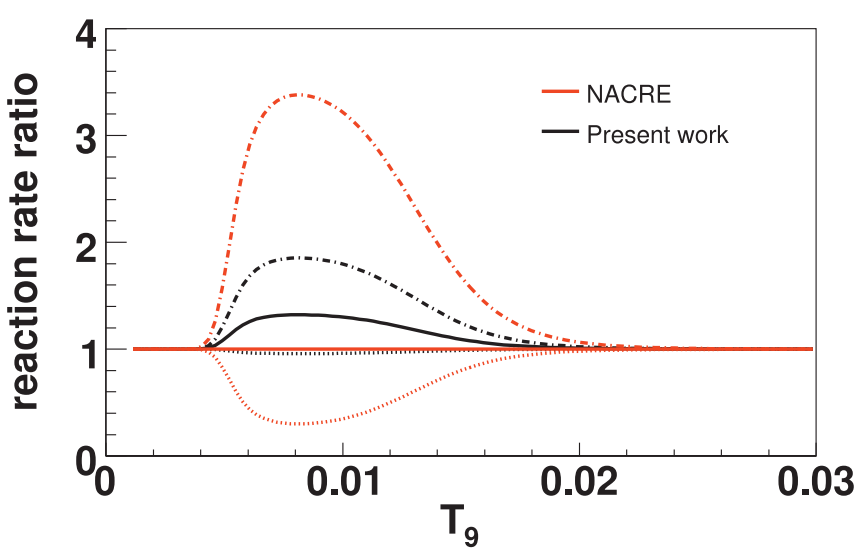

Fig. 3.5. Ratio of the La Cognata et al. (2008; labelled "Present work") and NACRE rates for ${ }^{18} \mathrm{O}(\mathrm{p}, \alpha){ }^{15} \mathrm{~N}$. The ratio of the upper and lower limits of the two sets of rates to the recommended values are also shown by dot-dashed and dotted curves, respectively. Original figure from La Cognata et al. (2008).

be too large for astrophysics purposes remain in the stellar rates. This situation results from the fact that the cross section is so small (approximately $10^{-17} \mathrm{~b}$ ) in stellar conditions that it will remain unreachable to direct measurement in the foreseeable future. Consequently, extrapolations from higher energies to the relevant Gamow window cannot be avoided. Their reliability is hampered by the special structural features of the ${ }^{16} \mathrm{O}$ nuclear levels, leading to various contributions to the $S$-factor (for a review, see e.g. Buchmann 2006).

The NACRE rates are not discussed here. We just review the post-NACRE information in some detail. In recent years, new experimental data have become available. They have been analysed extensively with the $R$-matrix method. Representative results are given by Kunz et al. (2002, 2001). The resulting reaction rates are based on $\gamma$-ray angular distribution measurements, and on a systematic description of the radiative capture data, elastic scattering data and $\alpha$-particle spectrum of the $\beta$-delayed $\alpha$-decay of ${ }^{16} \mathrm{~N}$. The derived rates are listed in Table 3.5. Following Kunz et al. (2002) they can be approximated by

$$
\begin{aligned}
N_{A}\langle\sigma v\rangle= & \frac{1.21 \times 10^{8}}{T_{9}^{2}\left(1+6.06 \times 10^{-2} T_{9}^{-2 / 3}\right)^{2}} \exp \left[-\frac{32.12}{T_{9}^{1 / 3}}-\left(\frac{T_{9}}{1.7}\right)^{2}\right] \\
& +\frac{7.4 \times 10^{8}}{T_{9}^{2}\left(1+0.47 T_{9}^{-2 / 3}\right)^{2}} \exp \left(-\frac{32.12}{T_{9}^{1 / 3}}\right) \\
& +\frac{1.53 \times 10^{4}}{T_{9}^{2 / 3}}\left(1+2 \times 10^{6} T_{9}^{1 / 3}\right) \exp \left(-\frac{38.534}{T_{9}^{1 / 3}}\right) .
\end{aligned}
$$


Table 3.5. Lower limits, recommended values and upper limits of the rates of ${ }^{12} \mathrm{C}(\alpha, \gamma){ }^{16} \mathrm{O}$ from the $R$-matrix analysis of Kunz et al. (2002).

\begin{tabular}{cccc}
\hline$T_{9}$ & lower & $N_{A}\langle\sigma v\rangle$ & upper \\
\hline 0.04 & $5.89 \mathrm{E}-31$ & $8.36 \mathrm{E}-31$ & $1.11 \mathrm{E}-30$ \\
0.05 & $3.65 \mathrm{E}-28$ & $5.20 \mathrm{E}-28$ & $6.93 \mathrm{E}-28$ \\
0.06 & $4.90 \mathrm{E}-26$ & $6.90 \mathrm{E}-26$ & $9.30 \mathrm{E}-26$ \\
0.07 & $2.39 \mathrm{E}-24$ & $3.42 \mathrm{E}-24$ & $4.57 \mathrm{E}-24$ \\
0.08 & $5.90 \mathrm{E}-23$ & $8.40 \mathrm{E}-23$ & $1.13 \mathrm{E}-22$ \\
0.09 & $8.80 \mathrm{E}-22$ & $1.26 \mathrm{E}-21$ & $1.69 \mathrm{E}-21$ \\
0.10 & $9.00 \mathrm{E}-21$ & $1.29 \mathrm{E}-20$ & $1.73 \mathrm{E}-20$ \\
0.11 & $6.80 \mathrm{E}-20$ & $9.80 \mathrm{E}-20$ & $1.32 \mathrm{E}-19$ \\
0.12 & $4.11 \mathrm{E}-19$ & $5.92 \mathrm{E}-19$ & $7.96 \mathrm{E}-19$ \\
0.13 & $2.04 \mathrm{E}-18$ & $2.93 \mathrm{E}-18$ & $3.95 \mathrm{E}-18$ \\
0.14 & $8.60 \mathrm{E}-18$ & $1.24 \mathrm{E}-17$ & $1.67 \mathrm{E}-17$ \\
0.15 & $3.18 \mathrm{E}-17$ & $4.58 \mathrm{E}-17$ & $6.18 \mathrm{E}-17$ \\
0.16 & $1.05 \mathrm{E}-16$ & $1.51 \mathrm{E}-16$ & $2.04 \mathrm{E}-16$ \\
0.18 & $8.60 \mathrm{E}-16$ & $1.24 \mathrm{E}-15$ & $1.68 \mathrm{E}-15$ \\
0.20 & $5.25 \mathrm{E}-15$ & $7.58 \mathrm{E}-15$ & $1.02 \mathrm{E}-14$ \\
0.25 & $1.93 \mathrm{E}-13$ & $2.79 \mathrm{E}-13$ & $3.79 \mathrm{E}-13$ \\
0.30 & $2.96 \mathrm{E}-12$ & $4.28 \mathrm{E}-12$ & $5.83 \mathrm{E}-12$ \\
0.35 & $2.60 \mathrm{E}-11$ & $3.75 \mathrm{E}-11$ & $5.11 \mathrm{E}-11$ \\
0.40 & $1.54 \mathrm{E}-10$ & $2.22 \mathrm{E}-10$ & $3.03 \mathrm{E}-10$ \\
0.45 & $6.90 \mathrm{E}-10$ & $9.90 \mathrm{E}-10$ & $1.35 \mathrm{E}-09$ \\
0.50 & $2.50 \mathrm{E}-09$ & $3.59 \mathrm{E}-09$ & $4.91 \mathrm{E}-09$ \\
0.60 & $2.06 \mathrm{E}-08$ & $2.95 \mathrm{E}-08$ & $4.04 \mathrm{E}-08$ \\
0.70 & $1.10 \mathrm{E}-07$ & $1.57 \mathrm{E}-07$ & $2.15 \mathrm{E}-07$ \\
0.80 & $4.36 \mathrm{E}-07$ & $6.19 \mathrm{E}-07$ & $8.48 \mathrm{E}-07$ \\
0.90 & $1.39 \mathrm{E}-06$ & $1.97 \mathrm{E}-06$ & $2.70 \mathrm{E}-06$ \\
1.00 & $3.78 \mathrm{E}-06$ & $5.32 \mathrm{E}-06$ & $7.29 \mathrm{E}-06$ \\
1.25 & $2.85 \mathrm{E}-05$ & $3.95 \mathrm{E}-05$ & $5.40 \mathrm{E}-05$ \\
1.50 & $1.40 \mathrm{E}-04$ & $1.91 \mathrm{E}-04$ & $2.59 \mathrm{E}-04$ \\
1.75 & $5.38 \mathrm{E}-04$ & $7.20 \mathrm{E}-04$ & $9.63 \mathrm{E}-04$ \\
2.00 & $1.75 \mathrm{E}-03$ & $2.29 \mathrm{E}-03$ & $3.00 \mathrm{E}-03$ \\
2.50 & $1.23 \mathrm{E}-02$ & $1.54 \mathrm{E}-02$ & $1.95 \mathrm{E}-02$ \\
3.00 & $5.55 \mathrm{E}-02$ & $6.81 \mathrm{E}-02$ & $8.33 \mathrm{E}-02$ \\
3.50 & $1.66 \mathrm{E}-01$ & $2.21 \mathrm{E}-01$ & $2.66 \mathrm{E}-01$ \\
4.00 & $4.82 \mathrm{E}-01$ & $5.86 \mathrm{E}-01$ & $6.98 \mathrm{E}-01$ \\
5.00 & $2.41 \mathrm{E}+00$ & $2.91 \mathrm{E}+00$ & $3.44 \mathrm{E}+00$ \\
6.00 & $7.63 \mathrm{E}+00$ & $1.07 \mathrm{E}+01$ & $1.26 \mathrm{E}+01$ \\
7.00 & $2.56 \mathrm{E}+01$ & $3.08 \mathrm{E}+01$ & $3.61 \mathrm{E}+01$ \\
8.00 & $5.88 \mathrm{E}+01$ & $7.08 \mathrm{E}+01$ & $8.30 \mathrm{E}+01$ \\
9.00 & $1.14 \mathrm{E}+02$ & $1.37 \mathrm{E}+02$ & $1.61 \mathrm{E}+02$ \\
10.00 & $1.93 \mathrm{E}+02$ & $2.32 \mathrm{E}+02$ & $2.72 \mathrm{E}+02$ \\
\hline & & & \\
\hline
\end{tabular}

Figure 3.6 compares the rates of Kunz et al. (2002) with those proposed by Caughlan \& Fowler (1988) (CF88), NACRE (recommended values) and Buchmann (1996). Note in particular that the CF88 and NACRE rates exhibit a different temperature dependence than the ones of Kunz et al. 

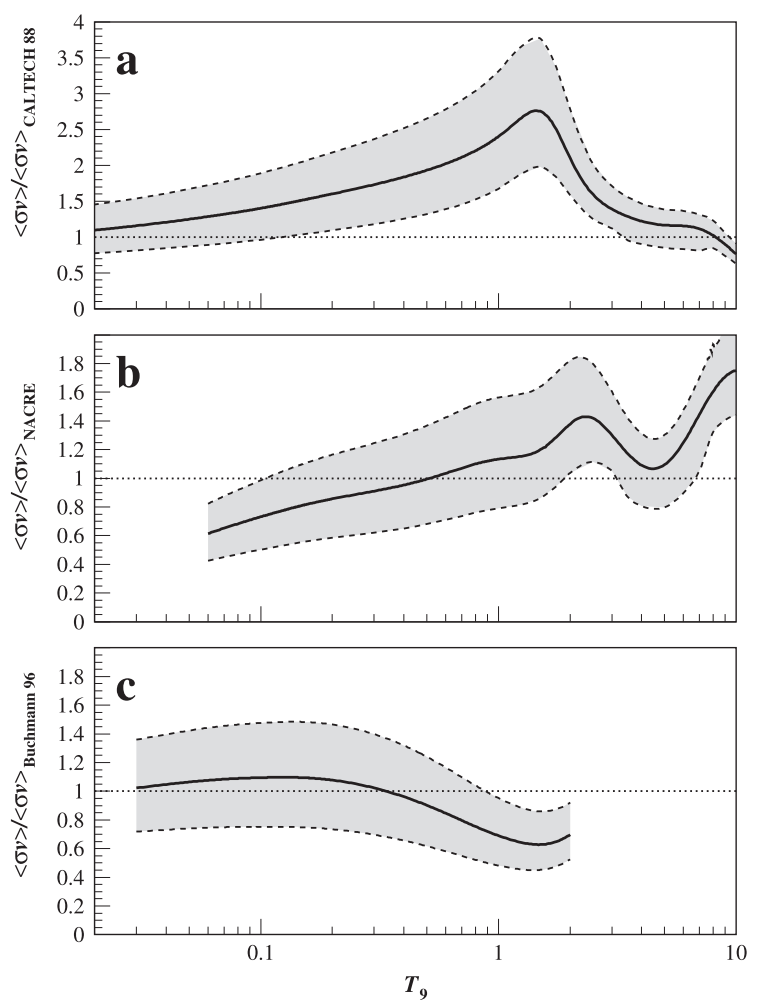

Fig. 3.6. Ratios of the rates for ${ }^{12} \mathrm{C}(\alpha, \gamma){ }^{16} \mathrm{O}$ proposed by Kunz et al. (2002) and Caughlan \& Fowler (1988) (CF88; panel a), NACRE recommended rates (panel b) (the contribution of cascade transitions is neglected; see e.g. Buchmann 2006 for a discussion of these transitions) and Buchmann (1996) (panel c). The solid curves are obtained with the recommended values of Table 3.5, and the shaded area are bounded by the ratios calculated with the lower and upper limits listed in this table. Original figure from Kunz et al. (2002).

A recent re-evaluation of the ${ }^{12} \mathrm{C}(\alpha, \gamma){ }^{16} \mathrm{O}$ rate based on the direct capture model (potential model) has been carried out by Katsuma (2008). Some rate values are listed in Table 3.6. They are compared in Figure 3.7 to the NACRE data. The two sets of predictions deviate from each other especially at $T_{9} \gtrsim 0.5$, where the higher rates obtained with the potential model are due to the inclusion of the contribution from cascade transitions to ${ }^{16} \mathrm{O}$ excited states that are neglected in NACRE. In contrast, any possible contribution from narrow resonances is neglected in the potential model rates, while they are included in NACRE. Comparing Figure 3.7 with Figure 3.6, the potential and $R$-matrix predictions show similar trends of the temperature dependence of the rates. However, the enhancement around $T_{9}=1-2$ compared to NACRE is much stronger in the potential than 
Table 3.6. The ${ }^{12} \mathrm{C}(\alpha, \gamma){ }^{16} \mathrm{O}$ rates calculated by Katsuma (2008) [KA08] with a potential model including the contribution of cascade transitions. The recommended NACRE rates are listed for comparison.

\begin{tabular}{cccccc}
\hline$T_{9}$ & {$[\mathrm{KA08}]$} & NACRE & $T_{9}$ & {$[\mathrm{KA08}]$} & NACRE \\
\hline 0.06 & $6.25 \mathrm{E}-26$ & $1.02 \mathrm{E}-25$ & 0.35 & $4.31 \mathrm{E}-11$ & $4.03 \mathrm{E}-11$ \\
0.07 & $3.18 \mathrm{E}-24$ & $4.98 \mathrm{E}-24$ & 0.40 & $2.60 \mathrm{E}-10$ & $2.31 \mathrm{E}-10$ \\
0.08 & $8.07 \mathrm{E}-23$ & $1.22 \mathrm{E}-22$ & 0.45 & $1.18 \mathrm{E}-09$ & $1.00 \mathrm{E}-09$ \\
0.09 & $1.23 \mathrm{E}-21$ & $1.80 \mathrm{E}-21$ & 0.50 & $4.32 \mathrm{E}-09$ & $3.52 \mathrm{E}-09$ \\
0.10 & $1.29 \mathrm{E}-20$ & $1.81 \mathrm{E}-20$ & 0.60 & $3.66 \mathrm{E}-08$ & $2.75 \mathrm{E}-08$ \\
0.11 & $9.91 \mathrm{E}-20$ & $1.35 \mathrm{E}-19$ & 0.70 & $2.01 \mathrm{E}-07$ & $1.40 \mathrm{E}-07$ \\
0.12 & $6.02 \mathrm{E}-19$ & $7.98 \mathrm{E}-19$ & 0.80 & $8.14 \mathrm{E}-07$ & $5.36 \mathrm{E}-07$ \\
0.13 & $3.02 \mathrm{E}-18$ & $3.89 \mathrm{E}-18$ & 0.90 & $2.66 \mathrm{E}-06$ & $1.66 \mathrm{E}-06$ \\
0.14 & $1.29 \mathrm{E}-17$ & $1.61 \mathrm{E}-17$ & 1.00 & $7.42 \mathrm{E}-06$ & $4.41 \mathrm{E}-06$ \\
0.15 & $4.80 \mathrm{E}-17$ & $5.86 \mathrm{E}-17$ & 1.25 & $5.96 \mathrm{E}-05$ & $3.19 \mathrm{E}-05$ \\
0.16 & $1.60 \mathrm{E}-16$ & $1.91 \mathrm{E}-16$ & 1.50 & $3.13 \mathrm{E}-04$ & $1.53 \mathrm{E}-04$ \\
0.18 & $1.32 \mathrm{E}-15$ & $1.53 \mathrm{E}-15$ & 1.75 & $1.26 \mathrm{E}-03$ & $5.76 \mathrm{E}-04$ \\
0.20 & $8.20 \mathrm{E}-15$ & $9.11 \mathrm{E}-15$ & 2.00 & $4.19 \mathrm{E}-03$ & $1.84 \mathrm{E}-03$ \\
0.25 & $3.09 \mathrm{E}-13$ & $3.21 \mathrm{E}-13$ & 2.50 & $2.85 \mathrm{E}-02$ & $1.27 \mathrm{E}-02$ \\
0.30 & $4.84 \mathrm{E}-12$ & $4.75 \mathrm{E}-12$ & 3.00 & $1.19 \mathrm{E}-01$ & $5.81 \mathrm{E}-02$ \\
\hline
\end{tabular}

in the $R$-matrix case. Further experiments in the energy range $E_{\text {c.m. }}=3-8 \mathrm{MeV}$ are in fact required to ascertain the reaction rate at $T_{9}>1$ (Kunz et al. 2002).

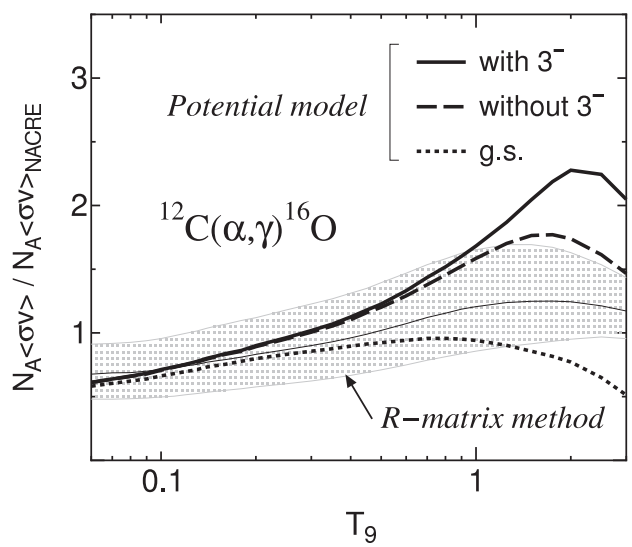

Fig. 3.7. Ratios of the rates of ${ }^{12} \mathrm{C}(\alpha, \gamma){ }^{16} \mathrm{O}$ calculated by Katsuma (2008) with a potential model to the NACRE rates. The solid, dashed and dotted lines are obtained with the use of the NACRE recommended rates, and with the inclusion of different transitions in the potential model, as indicated in the figure; the solid line refers to the rates of Table 3.6. The shaded band corresponds to the adoption of the rates of Table 3.6 and of the upper and lower limits of the NACRE rates. The label " $R$-matrix method" refers to the results of Kunz et al. (2002). 


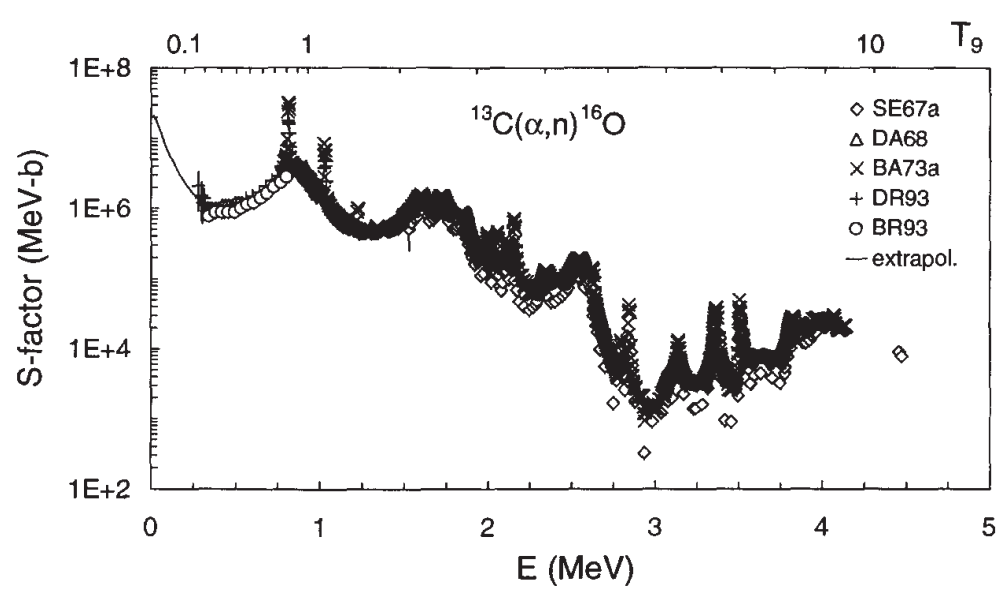

Fig. 3.8. $S$-factor for ${ }^{13} \mathrm{C}(\alpha, \mathrm{n}){ }^{16} \mathrm{O}$.

\subsubsection{5 ${ }^{13} \mathrm{C}(\alpha, \mathrm{n}){ }^{16} \mathrm{O}$}

About half of all heavy nuclides in the Universe are produced by the $s$-process, which involves a sequence of slow neutron captures and $\beta$-decays. The ${ }^{13} \mathrm{C}(\alpha, \mathrm{n})^{16} \mathrm{O}$ reaction is considered to be the main neutron source for the $s$-process in asymptotic giant branch (AGB) stars. The knowledge of its rate is thus necessary in order to evaluate the $s$-process efficiency in these stellar conditions.

At present, the direct measurements of the ${ }^{13} \mathrm{C}(\alpha, \mathrm{n}){ }^{16} \mathrm{O}$ rate have been limited to incident energies $E_{\text {c.m. }}>280 \mathrm{keV}$ (Drotleff et al. 1993) ${ }^{2}$. This is in excess of the Gamow peak energy which is around $200 \mathrm{keV}$ at temperatures $T_{9}=0.1$ close to those typical for the operation of the $s$-process in AGB stars.

The NACRE ${ }^{13} \mathrm{C}(\alpha, \mathrm{n}){ }^{16} \mathrm{O} S$-factor is shown in Figure 3.8. For $T_{9}<4$, it is based on experimental cross sections in the $E_{\text {c.m. }}=0.28-4.47 \mathrm{MeV}$ range. Below $E_{\text {c.m. }}=0.28 \mathrm{MeV}$, the $S$-factor extrapolation is obtained with the assumed contribution from the $1 / 2^{+}$subthreshold state at the excitation energy $E_{x}=$ $6.356 \mathrm{MeV}$ in ${ }^{17} \mathrm{O}$. This state is considered to originate from the $\alpha+{ }^{13} \mathrm{C}$ cluster structure with relative angular momentum $l=1$ (Descouvemont 1987; Dufour \& Descouvemont 2001). In NACRE, this structure is assumed to be well developed (i.e. to have a large $\alpha$-particle spectroscopic factor $S_{\alpha}$ ). In these conditions, the subthreshold state strongly influences the low-energy $S$-factor, and consequently the AGB star $s$-process efficiency.

In order to investigate the $\alpha+{ }^{13} \mathrm{C}$ component in the subthreshold state, indirect measurements of the ${ }^{13} \mathrm{C}\left({ }^{6} \mathrm{Li}, \mathrm{d}\right){ }^{17} \mathrm{O}$ and ${ }^{13} \mathrm{C}\left({ }^{7} \mathrm{Li}, \mathrm{t}\right){ }^{17} \mathrm{O}$ reactions have been performed by Kubono et al. (2003). From the analysis of their data with the help

\footnotetext{
${ }^{2}$ Note the recent direct measurement by Harissopulos et al. (2005) of the absolute cross section in the $E_{\alpha}=0.8$ to $8.0 \mathrm{MeV}$ range with an overall accuracy of $4 \%$.
} 


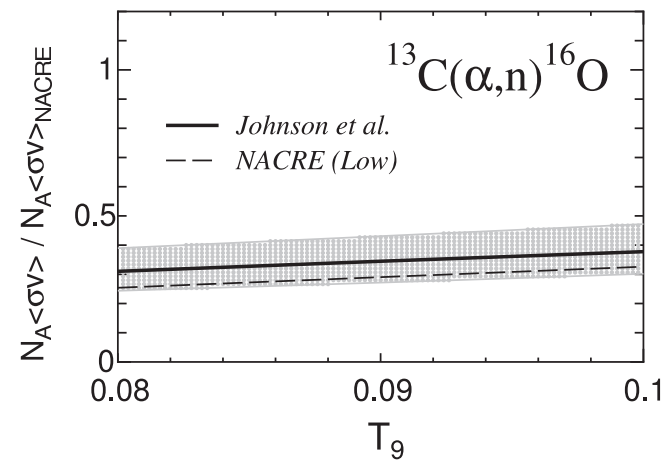

Fig. 3.9. Ratio of the ${ }^{13} \mathrm{C}(\alpha, \mathrm{n}){ }^{16} \mathrm{O}$ adopted rates obtained by the ANC method (Johnson et al. 2006) to the adopted NACRE ones (solid line). The dashed line corresponds to the ratio of the lower limit to the adopted NACRE rates. The shaded area shows the region between the ratios of the lower and upper limits of the rates of Johnson et al. (2006) to the adopted NACRE rates.

of the distorted waves Born approximation (DWBA), they have predicted $S_{\alpha}=$ 0.01 for this state. Compared to NACRE, this leads to a much reduced contribution of the subthreshold state to the ${ }^{13} \mathrm{C}(\alpha, \mathrm{n}){ }^{16} \mathrm{O}$ rate, and thus to a lowering of the efficiency of the ${ }^{13} \mathrm{C}$ neutron source.

The conclusions of Kubono et al. (2003) have been challenged by Keeley et al. (2003) who have re-analysed the same experimental data using the DWBA model complemented with the coupled reaction channel (CRC) model. The resulting $S_{\alpha} \approx 0.4$ is by far larger than the value reported by Kubono et al. (2003). A new measurement of ${ }^{13} \mathrm{C}\left({ }^{7} \mathrm{Li}, \mathrm{t}\right){ }^{17} \mathrm{O}$ has been performed recently by Pellegriti et al. (2008) and analysed with the DWBA model. The resulting $S_{\alpha}$, equal to $0.29 \pm 0.11$, is marginally in agreement with the value reported by Keeley et al. (2003).

The contribution from the subthreshold state has also been evaluated with the ANC method by Johnson et al. (2006) based on the low-energy differential cross section of ${ }^{13} \mathrm{C}\left({ }^{6} \mathrm{Li}, \mathrm{d}\right){ }^{17} \mathrm{O}\left(1 / 2^{+}\right)$measured in inverse kinematics. They derive the value $S=(2.5 \pm 0.7) \times 10^{6} \mathrm{MeV}$ b for the low-energy astrophysical $S$ factor, which is 10 times smaller than the one $\left(2.30 \times 10^{7} \mathrm{MeV}\right.$ b) adopted in NACRE. This leads to a reaction rate that is 3 times smaller than NACRE in the $T_{9}=0.08-0.1$ range, as shown in Figure 3.9. In fact, the adopted rates predicted by the ANC method correspond approximately to the lower limit of the NACRE rates (Table 3.7).

In summary, the ${ }^{13} \mathrm{C}(\alpha, n){ }^{16} \mathrm{O}$ rates are still quite uncertain in astrophysically relevant conditions. All the results obtained from the recent indirect measurements suggest, however, that the reaction rates in the $T_{9}=0.08-0.1$ range of relevance to the $s$-process are smaller than those adopted by NACRE. This relates to the fact that the $\alpha$-particle spectroscopic factor of the subthreshold $1 / 2^{+}$state seems to be $S_{\alpha}<0.4$, which implies that the $\alpha+{ }^{13} \mathrm{C}$ cluster structure may be less well developed than assumed in NACRE. 
Table 3.7. Rates of ${ }^{13} \mathrm{C}(\alpha, \mathrm{n}){ }^{16} \mathrm{O}$ predicted by the ANC method (Johnson et al. 2006) and reported in NACRE. For each temperature, the rates are obtained by multiplying the entries by $10^{\exp }$ (from Johnson et al. 2006).

\begin{tabular}{cccccccc}
\hline \multicolumn{4}{c}{ Johnson et al. } & \multicolumn{3}{c}{ NACRE } \\
\hline$T_{9}$ & Low & Adopt & High & Low & Adopt & High & exp \\
\hline 0.08 & 1.17 & 1.49 & 1.87 & 1.22 & 4.80 & 5.80 & -16 \\
0.09 & 1.90 & 2.41 & 3.01 & 2.03 & 6.99 & 8.45 & -15 \\
0.10 & 2.11 & 2.64 & 3.30 & 2.28 & 6.99 & 8.49 & -14 \\
\hline
\end{tabular}

Table 3.8. Adopted energies and strengths of the resonances contributing to ${ }^{14} \mathrm{~N}(\alpha, \gamma){ }^{18} \mathrm{~F}$ (from Görres et al. 2000).

\begin{tabular}{ccc}
\hline$E_{r}(\mathrm{MeV})$ & $J^{\pi}$ & $\omega \gamma(\mathrm{eV})$ \\
\hline 0.237 & $4^{+}$ & $2.6 \times 10^{-17}$ \\
0.446 & $1^{-}$ & $46 \times 10^{-6}$ \\
0.883 & $4^{+}$ & $21.1 \times 10^{-3}$ \\
1.088 & 3 & $7 \times 10^{-3}$ \\
1.189 & $1^{-}$ & 1.3 \\
1.258 & $1^{-}$ & 0.44 \\
\hline
\end{tabular}

Table 3.9. The ${ }^{14} \mathrm{~N}(\alpha, \gamma){ }^{18} \mathrm{~F}$ rates. They are obtained from the adoption of the resonance energies and strengths listed in Table 3.8. The direct capture component $N_{A}\langle\sigma v\rangle_{\mathrm{DC}}=1.01 \times 10^{9} T_{9}^{-2 / 3} \exp \left(-36.023 T_{9}^{-1 / 3}\right)($ Görres et al. 2000$)$ is added to the resonant contribution.

\begin{tabular}{lclclc}
\hline$T_{9}$ & $N_{A}\langle\sigma v\rangle$ & $T_{9}$ & $N_{A}\langle\sigma v\rangle$ & $T_{9}$ & $N_{A}\langle\sigma v\rangle$ \\
\hline 0.07 & $4.06 \mathrm{E}-28$ & 0.16 & $1.80 \mathrm{E}-13$ & 0.60 & $5.56 \mathrm{E}-04$ \\
0.08 & $4.50 \mathrm{E}-26$ & 0.18 & $5.49 \mathrm{E}-12$ & 0.70 & $1.99 \mathrm{E}-03$ \\
0.09 & $6.56 \mathrm{E}-24$ & 0.20 & $8.32 \mathrm{E}-11$ & 0.80 & $6.95 \mathrm{E}-03$ \\
0.10 & $1.38 \mathrm{E}-21$ & 0.25 & $1.05 \mathrm{E}-08$ & 0.90 & $2.36 \mathrm{E}-02$ \\
0.11 & $1.30 \mathrm{E}-19$ & 0.30 & $2.53 \mathrm{E}-07$ & 1.00 & $7.17 \mathrm{E}-02$ \\
0.12 & $5.76 \mathrm{E}-18$ & 0.35 & $2.36 \mathrm{E}-06$ & 1.25 & $6.31 \mathrm{E}-01$ \\
0.13 & $1.41 \mathrm{E}-16$ & 0.40 & $1.23 \mathrm{E}-05$ & 1.50 & $2.80 \mathrm{E}+00$ \\
0.14 & $2.17 \mathrm{E}-15$ & 0.45 & $4.35 \mathrm{E}-05$ & 1.75 & $8.02 \mathrm{E}+00$ \\
0.15 & $2.30 \mathrm{E}-14$ & 0.50 & $1.19 \mathrm{E}-04$ & 2.00 & $1.74 \mathrm{E}+01$ \\
\hline
\end{tabular}

\subsubsection{6 ${ }^{14} \mathrm{~N}(\alpha, \gamma){ }^{18} \mathrm{~F}$}

During He burning, the ${ }^{14} \mathrm{~N}$ abundantly produced by the CNO cycles is converted into ${ }^{18} \mathrm{O}$ via ${ }^{14} \mathrm{~N}(\alpha, \gamma){ }^{18} \mathrm{~F}\left(\mathrm{e}^{+} \nu\right)^{18} \mathrm{O}$. Following NACRE, resonances located at $E_{r}=$ $0.237,0.435,0.883,1.088,1.189$ and $1.258 \mathrm{MeV}$ contribute significantly to the rate of ${ }^{14} \mathrm{~N}(\alpha, \gamma){ }^{18} \mathrm{~F}$ below $T_{9}=2$. This resonance contribution has been re-investigated by Görres et al. (2000). Their adopted resonances and strengths are listed in Table 3.8. We use these data to calculate the reaction rates of Table 3.9. The rates in the $T_{9}=0.1-0.5$ range are dominated by the narrow $1^{-}$resonance at 


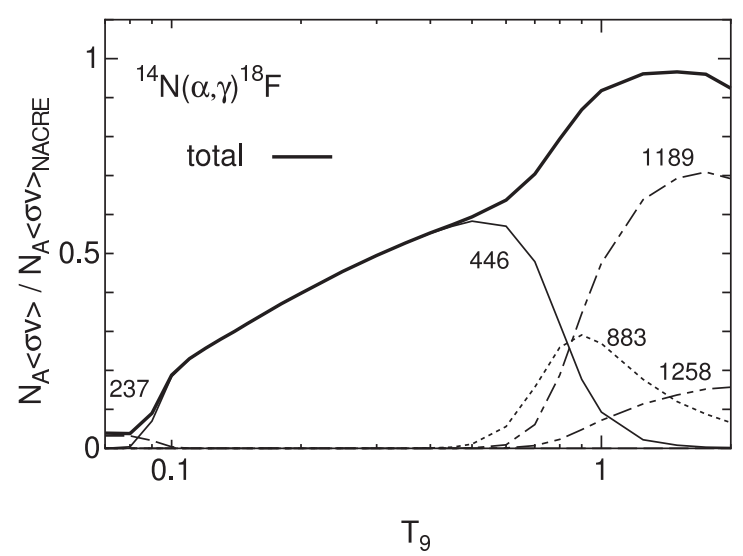

Fig. 3.10. Ratio of the rates derived from Görres et al. (see Table 3.9) to the adopted NACRE ones for ${ }^{14} \mathrm{~N}(\alpha, \gamma){ }^{18} \mathrm{~F}$. The bold solid curve refers to the total reaction rates, while the curves labelled with a resonance energy (in keV) correspond to the contribution of individual resonances.

$E_{r}=446 \mathrm{keV}$. In contrast, the resonances above $E_{r}=1.373 \mathrm{MeV}$ (see NACRE) have no impact on the rates below $T_{9}=2$, and are neglected here. The nonresonant direct capture component proposed by Görres et al. (2000) is added to the resonance contribution. It is influential only in the approximate $T_{9}=$ $0.1-0.5$ range.

Figure 3.10 shows the ratio of the new rates to the adopted NACRE ones. The new rates are seen to be $2-5$ times smaller in the $T_{9}=0.1-0.5$ range. This change comes mainly from the revised energies and strengths of the lowest two resonances.

\subsubsection{7 ${ }^{18} \mathrm{O}(\alpha, \gamma){ }^{22} \mathrm{Ne}$}

The burning of ${ }^{14} \mathrm{~N}$ into ${ }^{18} \mathrm{O}$ (Sect. 3.1.2.6) is followed by ${ }^{18} \mathrm{O}(\alpha, \gamma){ }^{22} \mathrm{Ne}$. This opens the possibility of neutron production by ${ }^{22} \mathrm{Ne}(\alpha, \mathrm{n}){ }^{25} \mathrm{Mg}$, which is considered to be responsible for the $s$-process in massive stars.

Below $T_{9}=1$, the ${ }^{18} \mathrm{O}(\alpha, \gamma){ }^{22}$ Ne rate is dominated by the contribution of resonances. In particular, the rate at temperatures close to typical $s$-process ones $\left(T_{9} \lesssim 0.3\right)$ is dominated by resonances with energies below $490 \mathrm{keV}$. Dababneh et al. (2003) have conducted a post-NACRE re-investigation of the low energy resonances, and their measurements lead to the reaction rates listed in Table 3.10. These rates are slightly reduced compared to NACRE at $T_{9}=0.1-0.3$ (see also Fig. 3.11), and the uncertainties are appreciably reduced. For $T_{9} \leq 6$, the 
Table 3.10. The ${ }^{18} \mathrm{O}(\alpha, \gamma){ }^{22} \mathrm{Ne}$ adopted rates from Dababneh et al. (2003) and from NACRE.

\begin{tabular}{lcclcc}
\hline$T_{9}$ & DA03 & NACRE & $T_{9}$ & DA03 & NACRE \\
\hline 0.07 & $1.53 \mathrm{E}-24$ & $1.53 \mathrm{E}-24$ & 0.25 & $2.55 \mathrm{E}-09$ & $2.95 \mathrm{E}-09$ \\
0.08 & $5.07 \mathrm{E}-23$ & $5.08 \mathrm{E}-23$ & 0.3 & $6.70 \mathrm{E}-08$ & $7.33 \mathrm{E}-08$ \\
0.09 & $8.86 \mathrm{E}-22$ & $9.20 \mathrm{E}-22$ & 0.35 & $8.86 \mathrm{E}-07$ & $9.38 \mathrm{E}-06$ \\
0.1 & $2.32 \mathrm{E}-20$ & $2.73 \mathrm{E}-20$ & 0.4 & $6.86 \mathrm{E}-06$ & $7.16 \mathrm{E}-06$ \\
0.11 & $8.83 \mathrm{E}-19$ & $1.09 \mathrm{E}-18$ & 0.45 & $3.50 \mathrm{E}-05$ & $3.63 \mathrm{E}-05$ \\
0.12 & $2.19 \mathrm{E}-17$ & $2.72 \mathrm{E}-17$ & 0.5 & $1.30 \mathrm{E}-04$ & $1.35 \mathrm{E}-04$ \\
0.13 & $3.38 \mathrm{E}-16$ & $4.20 \mathrm{E}-16$ & 0.6 & $9.44 \mathrm{E}-04$ & $9.74 \mathrm{E}-04$ \\
0.14 & $3.51 \mathrm{E}-15$ & $4.36 \mathrm{E}-15$ & 0.7 & $4 . \mathrm{E}-03$ & $3.94 \mathrm{E}-03$ \\
0.15 & $2.65 \mathrm{E}-14$ & $3.29 \mathrm{E}-14$ & 0.8 & $1.1 \mathrm{E}-02$ & $1.11 \mathrm{E}-02$ \\
0.16 & $1.55 \mathrm{E}-13$ & $1.92 \mathrm{E}-13$ & 0.9 & $2.4 \mathrm{E}-02$ & $2.46 \mathrm{E}-02$ \\
0.18 & $2.94 \mathrm{E}-12$ & $3.62 \mathrm{E}-12$ & 1. & $4.5 \mathrm{E}-02$ & $4.63 \mathrm{E}-02$ \\
0.2 & $3.11 \mathrm{E}-11$ & $3.79 \mathrm{E}-11$ & & & \\
\hline
\end{tabular}

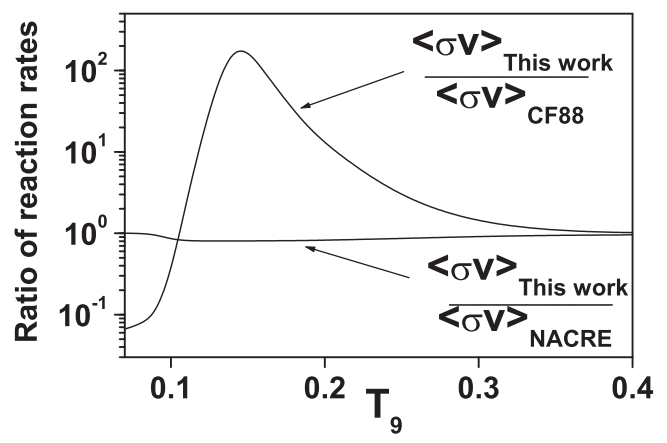

Fig. 3.11. Ratio of the Dababneh et al. (2003) ("This work") and NACRE adopted rates for ${ }^{18} \mathrm{O}(\alpha, \gamma)^{22} \mathrm{Ne}$. The ratio of the Dababneh et al. (2003) and Caughlan \& Fowler (1988) [CF88] rates is also shown. Figure from Dababneh et al. (2003).

Dababneh et al. $(2003){ }^{18} \mathrm{O}(\alpha, \gamma){ }^{22} \mathrm{Ne}$ rate can be approximated by

$$
\begin{aligned}
N_{A}\langle\sigma v\rangle= & 1.95 \times 10^{-13} T_{9}^{-3 / 2} \exp \left(-2.069 / T_{9}\right) \\
& +1.248 \times 10^{-2} T_{9}^{-3 / 2} \exp \left(-4.462 / T_{9}\right) \\
& +1.845 \times 10^{-2} T_{9}^{-3 / 2} \exp \left(-5.373 / T_{9}\right) \\
& +5.95 T_{9}^{-3 / 2} \exp \left(-6.285 / T_{9}\right)+12.74 T_{9}^{-3 / 2} \exp \left(-7.119 / T_{9}\right) \\
& +31.19 T_{9}^{-3 / 2} \exp \left(-7.287 / T_{9}\right) \\
& +3.22 \times 10^{5} T_{9}^{-1 / 2} \exp \left(-21.801 / T_{9}\right)
\end{aligned}
$$




\subsubsection{Other charged-particle reactions involving stable oxygen isotopes}

As already emphasised in the introduction of this section, several charged-particle induced reactions which involve the stable oxygen isotopes directly or through the decay of an unstable isobar are not discussed here, as their rates have not been revised since the publication of NACRE. The reader is referred to Angulo et al. (1999) and to the website http://www.astro.ulb.ac.be for detailed information on the rates of the reactions ${ }^{16} \mathrm{O}(\mathrm{p}, \gamma){ }^{17} \mathrm{~F},{ }^{14} \mathrm{~N}(\alpha, \mathrm{n}){ }^{17} \mathrm{~F},{ }^{16} \mathrm{O}(\alpha, \gamma){ }^{20} \mathrm{Ne}$, ${ }^{17} \mathrm{O}(\alpha, \mathrm{n}){ }^{20} \mathrm{Ne},{ }^{18} \mathrm{O}(\alpha, \mathrm{n}){ }^{21} \mathrm{Ne},{ }^{19} \mathrm{~F}(\mathrm{p}, \alpha){ }^{16} \mathrm{O}$ and ${ }^{20} \mathrm{Ne}(\mathrm{p}, \alpha){ }^{17} \mathrm{~F}$.

\subsubsection{A brief analysis of the impact of nuclear uncertainties on the stellar oxygen yields}

As it is well known, the cold $\mathrm{CNO}$ cycles produce ${ }^{4} \mathrm{He}$, while almost the entire initial $\mathrm{C}, \mathrm{N}$ and $\mathrm{O}$ content of the $\mathrm{H}$-burning zones turns into ${ }^{14} \mathrm{~N}$ as a result of the relative slowness of ${ }^{14} \mathrm{~N}(\mathrm{p}, \gamma){ }^{15} \mathrm{O}$ with respect to the other relevant reactions. These cycles also modify the isotopic composition of the residual oxygen.

The cold CNO cycles comprise branching points at ${ }^{15} \mathrm{~N},{ }^{17} \mathrm{O}$ and ${ }^{18} \mathrm{O}$ (see Fig. 3.1). In terms of the NACRE rates, their main characteristics may be very briefly summarised as follows: (i) at $T_{6}=25\left(T_{6}\right.$ is the temperature expressed in $\left.10^{6} \mathrm{~K}\right),{ }^{15} \mathrm{~N}(\mathrm{p}, \alpha){ }^{12} \mathrm{C}$ is 1000 times faster than ${ }^{15} \mathrm{~N}(\mathrm{p}, \gamma){ }^{16} \mathrm{O}$, and the $\mathrm{CN}$ cycle reaches equilibrium already before $10^{-3}$ of the initial protons have been burned; (ii) ${ }^{17} \mathrm{O}(\mathrm{p}, \alpha){ }^{14} \mathrm{~N}$ and ${ }^{17} \mathrm{O}(\mathrm{p}, \gamma){ }^{18} \mathrm{~F}$ are the competing ${ }^{17} \mathrm{O}$ destruction reactions and (iii) ${ }^{18} \mathrm{O}(\mathrm{p}, \gamma){ }^{19} \mathrm{~F}$ and ${ }^{18} \mathrm{O}(\mathrm{p}, \alpha){ }^{15} \mathrm{~N}$ compete to destroy ${ }^{18} \mathrm{O}$. At the relevant temperatures, NACRE predicts that ${ }^{18} \mathrm{O}(\mathrm{p}, \gamma){ }^{19} \mathrm{~F}$ is roughly 1000 times slower than ${ }^{18} \mathrm{O}(\mathrm{p}, \alpha){ }^{15} \mathrm{~N}$. Finally, let us note that any important leakage out of the $\mathrm{CNO}$ cycles to ${ }^{20} \mathrm{Ne}$ is prevented by the fact that ${ }^{19} \mathrm{~F}(\mathrm{p}, \alpha){ }^{16} \mathrm{O}$ is always much faster than ${ }^{19} \mathrm{~F}(\mathrm{p}, \gamma){ }^{20} \mathrm{~F}$. Certainly, at least some of the features mentioned above may be affected to some extent by standing uncertainties in some key rates.

The impact of the reaction rate uncertainties on the CNO yields has been analysed in the framework of parameterised astrophysics conditions or of realistic stellar models. We just illustrate some results from selected studies. Figure 3.12 shows the abundances of the $\mathrm{O}$ isotopes obtained under the assumption of constant H-burning density $\rho=100 \mathrm{~g} \mathrm{~cm}^{-3}$ and at two different constant temperatures, $T_{6}=25$ and 55 . The curves are constructed by combining, in every possible way, the NACRE lower and upper limits of all the relevant reaction rates. One "reference" abundance calculation is also performed with all the recommended NACRE rates. The initial abundances are the solar values of Anders \& Grevesse (1989). In spite of its highly simplistic nature, this analysis provides results that are of reasonable qualitative value, as testified by comparison with detailed stellar model predictions.

Figure 3.12 confirms the well-known drastic dependence of the O isotopic composition on the burning temperature. In particular, ${ }^{17} \mathrm{O}$ is produced at $T_{6} \lesssim 25$, but is destroyed at higher temperatures. This has the important consequence that 


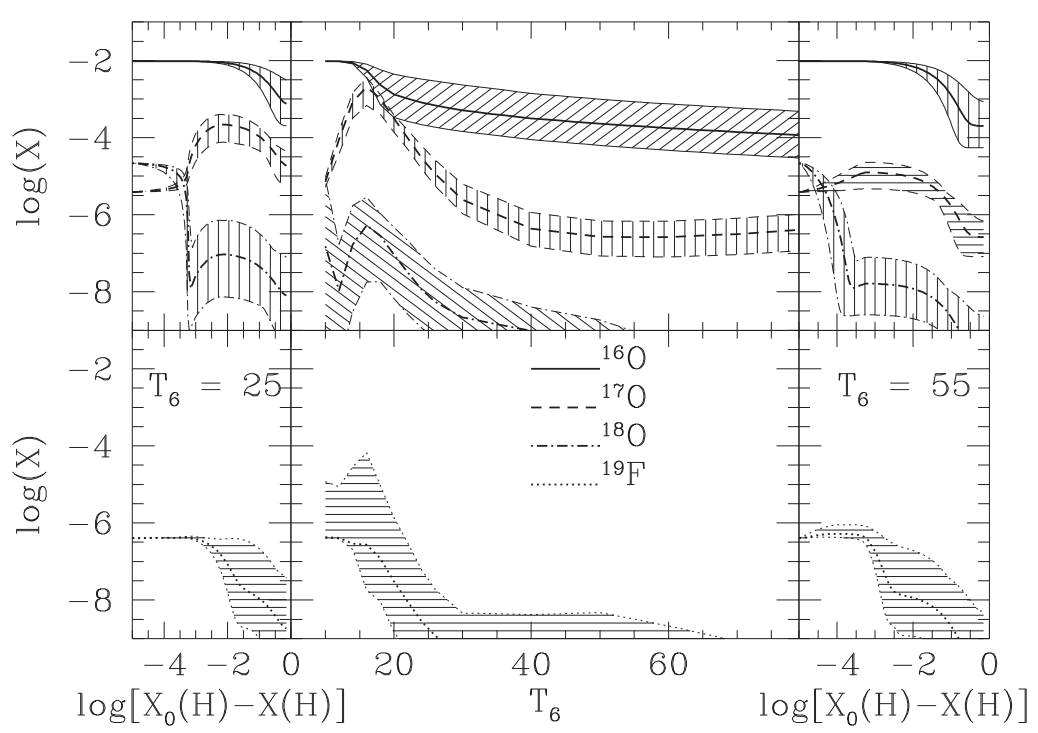

Fig. 3.12. Left and right panels: time variations of the mass fractions of the stable $O$ isotopes versus the amount of hydrogen burned at constant density $\rho=100 \mathrm{~g} \mathrm{~cm}^{-3}$ and constant temperatures $T_{6}=25$ and 55. The $\mathrm{H}$ mass fraction is labelled $X(\mathrm{H})$, with the subscript 0 referring to its initial value. Data for ${ }^{19} \mathrm{~F}$ are included. Middle panel: mass fractions of the same nuclides at $\mathrm{H}$ exhaustion $\left[\mathrm{X}(\mathrm{H})=10^{-5}\right]$ as a function of $T_{6}$. The shaded areas delineate the uncertainties resulting from the NACRE reaction rates. Figure from Arnould et al. (2003).

the amount of ${ }^{17} \mathrm{O}$ emerging from the $\mathrm{CNO}$ cycles, and eventually transported to the stellar surface, is a steep function of the stellar mass. This conclusion could get some support from the observation of a large spread in the oxygen isotopic ratios at the surface of red giant stars of somewhat different masses. Figure 3.12 also demonstrates that the oxygen isotopic composition cannot as yet be fully reliably predicted at a given temperature as a result of the cumulative uncertainties associated with the different production and destruction rates. This situation prevents a fully meaningful comparison to be made with spectroscopic data, and even more so, any firm conclusion to be drawn from models for the chemical evolution of galaxies. Figure 3.12 is complemented with Table 3.11 which illustrates uncertainties of purely nuclear origins associated to the operation of the CNO cycles in various detailed model stars at different stages of their evolution. Of course, the results displayed in Figure 3.12 and in Table 3.11 must be updated with the adoption of the revised rates discussed above. The conclusions are not likely to be drastically different from those mentioned above.

An extended sensitivity analysis of the nova properties and yields to reaction rate uncertainties has also been conducted over the last decade (Starrfield et al. 2009, and references therein). These explosions involve the hot pp, CNO and $\mathrm{NeNa}$ or $\mathrm{MgAl}$ chains of $\mathrm{H}$ burning, in which proton captures by unstable nuclides 

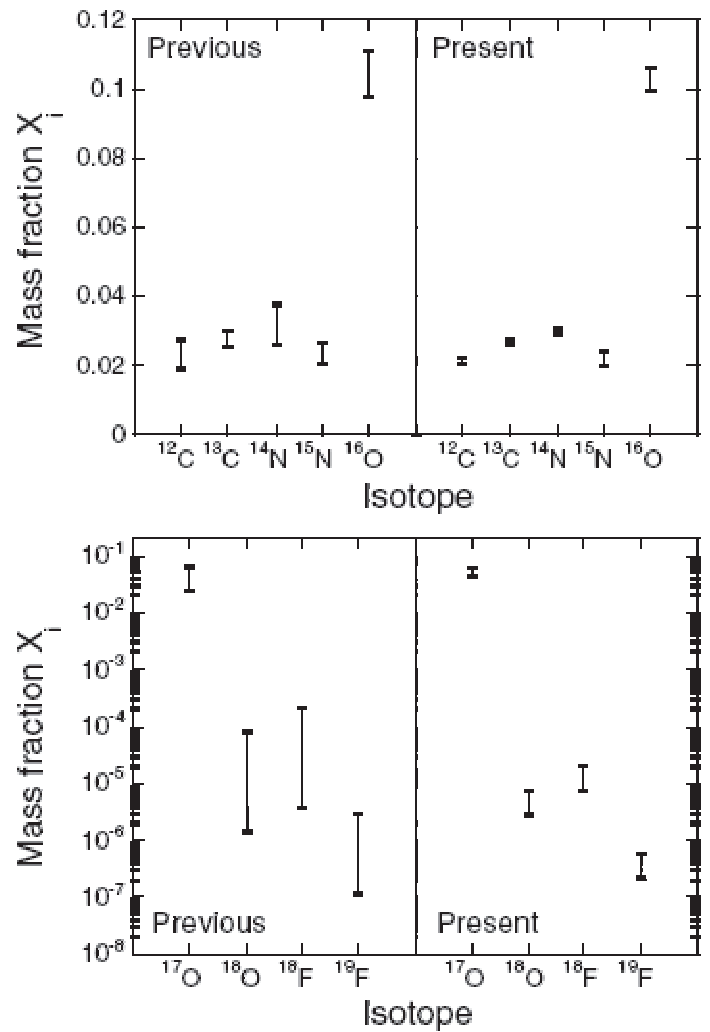

Fig. 3.13. Variations of the predicted $\mathrm{C}, \mathrm{N}$ and $\mathrm{O}$ isotopic mass fractions in the ejecta of a specific nova model. The abundances in the left panels result from the use of the recommended NACRE ${ }^{17} \mathrm{O}(\mathrm{p}, \gamma){ }^{18} \mathrm{~F}$ and ${ }^{17} \mathrm{O}(\mathrm{p}, \alpha){ }^{14} \mathrm{~N}$ rates. Those on the right are predicted with the rates of Fox et al. (2004). Figure adopted from Fox et al. (2004).

compete with $\beta$-decays. The hot $\mathrm{CNO}$ also involves reactions making up the cold mode (see Fig. 3.1) such as ${ }^{17} \mathrm{O}(\mathrm{p}, \gamma){ }^{18} \mathrm{~F},{ }^{17} \mathrm{O}(\mathrm{p}, \alpha){ }^{14} \mathrm{~N}$ and ${ }^{18} \mathrm{~F}(\mathrm{p}, \alpha){ }^{15} \mathrm{O}$. Figure 3.13 shows the changes in the abundances of the oxygen isotopes from a specific nova model that result from the use of the ${ }^{17} \mathrm{O}$ proton capture rates from Fox et al. (2004) instead of the NACRE values (see Sect. 3.1.2.2). This may not be, however, the last word about the ${ }^{17} \mathrm{O}$ nova yields, since using their own rates Chafa et al. (2005) obtain abundances closer to those derived using the NACRE rates than to those derived using Fox et al. (2004) rates.

\subsubsection{Neutron captures involving oxygen isotopes}

Stellar radiative neutron capture rates by ${ }^{15} \mathrm{~N},{ }^{16} \mathrm{O}$ and ${ }^{18} \mathrm{O}$ can be found in the compilation by Bao et al. (2000). For ${ }^{17} \mathrm{O}$, only an estimate by Wagoner (1969) is 
Table 3.11. Oxygen isotopic composition at the surface of stars of initial solar content and with different masses $M$ after convective transport of part of the central H-burning ashes. The isotopic ratios and the quoted uncertainties result from the use of the recommended NACRE rates of $\mathrm{H}$ burning through the CNO mode, and of their lower and upper limits provided by NACRE. Table from Stoesz \& Herwig (2003).

\begin{tabular}{lll}
\hline$M\left(M_{\odot}\right)$ & ${ }^{16} \mathrm{O} /{ }^{17} \mathrm{O}$ & ${ }^{16} \mathrm{O} /{ }^{18} \mathrm{O}$ \\
\hline 1.0 & $2410_{-16}^{+13}$ & $469_{-12}^{+46}$ \\
1.5 & $1260_{-130}^{+180}$ & $545_{-20}^{+70}$ \\
2.0 & $115_{-20}^{+38}$ & $565_{-20}^{+95}$ \\
Initial & 2465 & 442 \\
\hline
\end{tabular}

available. As shown by Rayet \& Hashimoto (2000), ${ }^{16} \mathrm{O}$ can become an important neutron poison in stars with low enough metallicity where it reduces the efficiency of the $s$-process.

\subsubsection{Conclusions}

Since the NACRE compilation, much effort has been put into the improvement of our knowledge of the rates of a variety of reactions involved in the stellar production or destruction of the stable oxygen isotopes. In spite of some progress, nuclear uncertainties remain which add up to those of purely astrophysical origin. To some extent, this situation hampers both a fully reliable comparison with relevant spectroscopic information and a quantitative evaluation of the merits of the different model stars.

\subsection{Stellar nucleosynthesis}

\subsubsection{Stellar evolution in a nutshell}

During the longest part of their lives, stars are in hydrostatic equilibrium. A star element in equilibrium undergoes a gravitational force balanced by a pressure gradient. In a normal star, pressure depends on temperature, and therefore the existence of a pressure gradient implies the buildup of a temperature gradient. The higher the temperature, the greater the quantity of energy contained per unit volume in the radiation field. Since the central parts are hotter than the outer ones due to the temperature gradient resulting from hydrostatic equilibrium, energy flows outwards from the inner parts of the star, and thus the star loses energy continually. Interestingly, the more efficiently the energy is evacuated from the central regions where it is produced, the hotter these central regions become! Indeed, if the central regions lose energy, the temperature gradient will become weaker and they will slowly contract, becoming warmer! Stars (made of perfect gas) are thus systems with a global negative specific heat.

Stars evolve because they continually lose energy to maintain hydrostatic equilibrium or, in other words, to balance the gravitational force. Note that these 
logical deductions do not involve any particular source of energy. Luminosity is a direct consequence of hydrostatic equilibrium and not of the nuclear reactions that occur in the stellar interior. Of course nuclear reactions are important and we are going to see how below.

\subsubsection{The main energy reservoirs}

The energy which flows from the stellar interior and is lost at the surface has of course to be extracted in one way or another from the stellar material. These mechanisms of energy extraction are responsible for the evolution of the star.

There are actually two main sources of energy in a star. First, a star can extract energy from the gravitational potential through a global (macroscopic) contraction. Second, a star can also release energy by the thermonuclear reactions which take place in its central regions where the temperature and density are adequate for such processes to occur (microscopic contractions).

These two processes for producing energy have different characteristic timescales. If the Sun had only the gravitational energy source, its lifetime would be of the order of a few tens of million years, instead a simple estimate gives a lifetime of ten billion years when nuclear sources are taken into account. Only the latter value is in agreement with what we know about the past history of the Earth and the apparition of life on its surface. Therefore the presence of the nuclear source is important not to explain the luminosity of stars (stars could shine even without hosting nuclear active regions), but to explain why they can shine for very long periods of time. Besides this energetic aspect, nuclear reactions are of course the processes through which chemical elements are synthesised in stellar interiors, and play a key role in the long chain of events that occur from the Big Bang to the apparition of living bodies.

The evolution of a star can be viewed as a succession of phases whereby the energy is mainly produced by either nuclear reactions or contraction. When the star has burned all the nuclear fuel available in its central regions, in order to maintain the luminosity required by the hydrostatic equilibrium, it has to produce energy via contraction until new central conditions are reached that are adequate for the ignition of new nuclear reactions. This succession of contraction periods will increase both the central temperature and density as can be seen in Figure 3.14. At a given point (which depends mainly on the initial mass of the star), the central regions can become sensitive to degeneracy effects.

\subsubsection{Perfect gas and degenerate gas}

Let us recall that degeneracy pressure results from Pauli Exclusion Principle: only two fermions of spin one-half, e.g. electrons, neutrons or neutrinos, may occupy the same spatial quantum state. Two particles are in two different quantum states if the product of their differences in position $\Delta x$ and momentum $\Delta p$ is superior to the Planck constant. An increase of the density restricts the domain for the positions and thus reduces $\Delta x$. The Exclusion Principle implies therefore that certain particles will acquire very large impulses, much greater than they would 


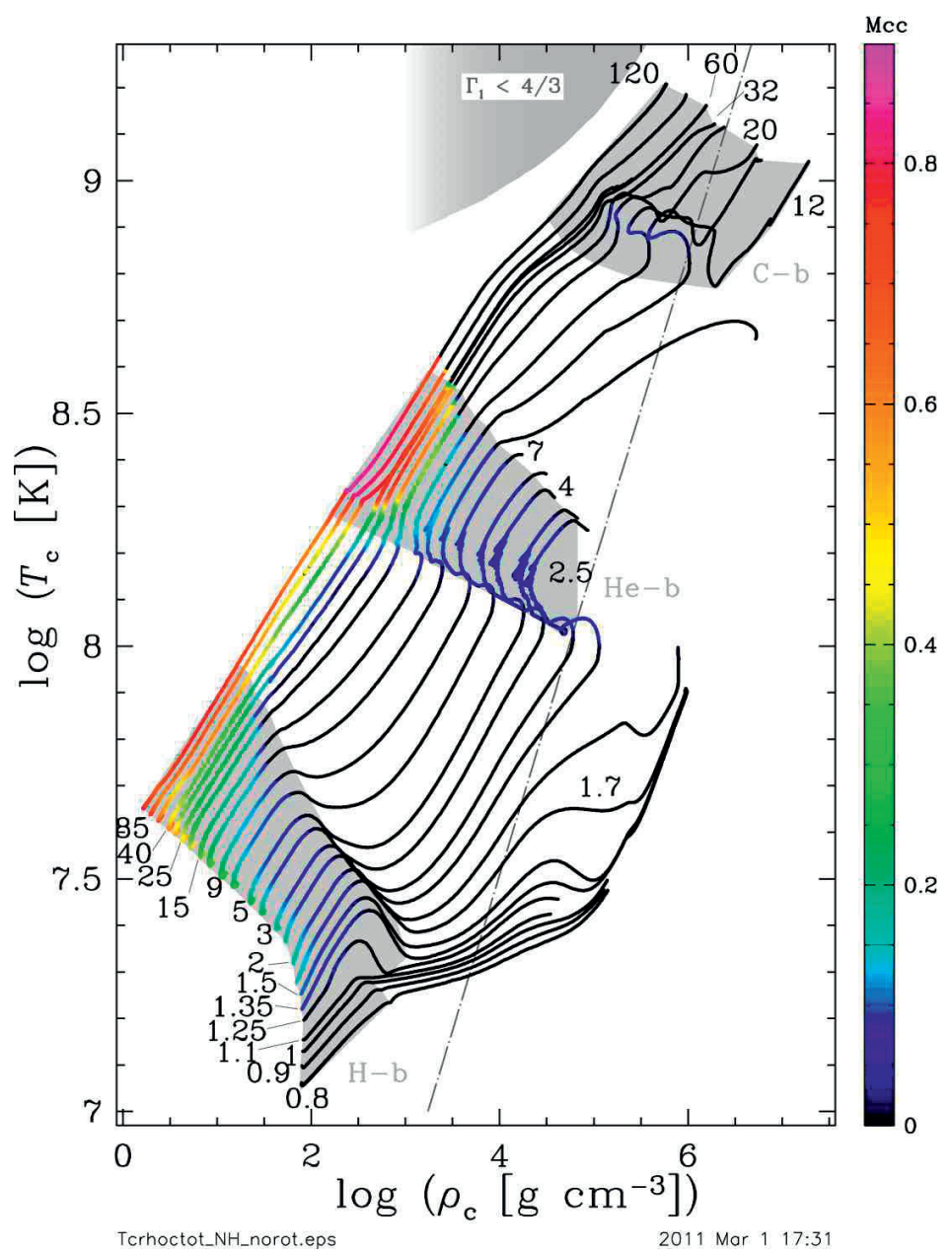

Fig. 3.14. Evolutionary tracks for rotating stellar models at a metallicity $Z=0.014$ in the $\log T_{\mathrm{c}}-\log \rho_{\mathrm{c}}$ plane. Grey zones along the tracks indicate the positions of stars during the core $\mathrm{H}-$, He- and C-burning phases. The region of pair instability is indicated by $\Gamma_{1}<4 / 3$. The line where the Fermi energy of the electron is equal $2 \mathrm{kT}$ is indicated (long dashed-dotted line). The colours specify the mass fraction of the convective cores. Figure from Ekström et al. (2011).

by thermal agitation. These particles with large velocities exert a pressure that is not thermal in origin and depends only on density.

Depending on whether the stellar material is degenerate or not, the two main sources of energy, contraction and nuclear reactions, have very different behaviours. In non-degenerate conditions, nuclear reactions are stable and contraction implies 
an increase of the central temperature. In degenerate conditions, nuclear reactions are explosive and contraction may produce a cooling of the medium.

Let us first consider a uniform contraction of a mass $M$. For such case a variation in radius $\Delta R$ corresponds to variations in pressure $\Delta P$ and density $\Delta \rho$ so that we have the following relations:

$$
\frac{\Delta P}{P}=-4 \frac{\Delta R}{R}, \text { and } \frac{\Delta \rho}{\rho}=-3 \frac{\Delta R}{R} .
$$

The first equality is deduced from the hydrostatic equilibrium equation and the second from the continuity equation. From these two relations, we can write

$$
\Delta \ln P=\frac{4}{3} \Delta \ln \rho .
$$

Let us now express the equation of state as

$$
\Delta \ln \rho=\alpha \Delta \ln P-\delta \Delta \ln T,
$$

where $\alpha$ and $\delta$ are defined by $\alpha=\left(\frac{\partial \ln \rho}{\partial \ln P}\right)_{T, \mu}$ and $\delta=-\left(\frac{\partial \ln \rho}{\partial \ln T}\right)_{P, \mu}$, and where $\mu$, the mean molecular weight, is supposed to remain constant. From these two relations, by eliminating $\Delta P$, one obtains the following relation between a variation in $\log \mathrm{T}$ and $\log \rho$ :

$$
\Delta \ln T=\left(\frac{4 \alpha-3}{3 \delta}\right) \Delta \ln \rho .
$$

For a perfect gas law we have $\alpha=\delta=1$. Therefore an increase of, for instance, $3 \%$ in density implies an increases of $1 \%$ in temperature.

On the other hand, if the gas is completely degenerate and non-relativistic, the relation between the degenerate electronic gas pressure and density is given by

$$
P \propto \rho^{5 / 3}
$$

In the $\log T_{\mathrm{c}}$ versus $\log \rho_{\mathrm{c}}$ diagram, where $T_{\mathrm{c}}$ is the central temperature and $\rho_{\mathrm{c}}$ is the central density, the region where the perfect gas electron pressure equals that of the degenerate gas is a line with slope $2 / 3$. The track of a slowly contracting star in the $\log T_{\mathrm{c}}-\log \rho_{\mathrm{c}}$ diagram is a straight line with slope $1 / 3$ (see Eq. (3.5) with $\alpha=\delta=1$ ). Thus eventually a star will cross the frontier between the perfect and degenerate gas. For a degenerate, non-relativistic gas, $\alpha=3 / 5$ and $\delta=0$. In this case, Equation (3.5) is no longer valid, but if during the course of evolution when the central conditions shift from the non-degenerate to the degenerate regime $\alpha$ becomes less than $3 / 4$ before $\delta$ is equal to zero, a contraction can then produce cooling! This can be understood as due to the fact that, in order to allow electrons to occupy still higher energy states, some energy has to be extracted from the nondegenerate nuclei which, as a consequence, cool down. 


\subsubsection{Evolution under non-degenerate and degenerate conditions}

Let us first consider non-degenerate conditions. Let us imagine that for whatever reason an excess of energy is produced in the centre of the star. This will produce the heating of matter. When the perfect gas law prevails, an increase of temperature will produce an increase of pressure and therefore an expansion. This implies an increase of the potential energy and, through the virial theorem, a decrease of the internal energy. Therefore the temperature decreases as well as the nuclear reaction rates. We see that, in perfect gas conditions, there is a negative feedback which stops the runaway. The nuclear reactions are stable when the perfect gas law prevails.

When matter is degenerate, the behaviour is quite different. The excess of energy produced at the centre, which implies a temperature increase, no longer provokes an expansion, since there is no longer a coupling between pressure and temperature. The nuclear reaction rates increase, new excesses of energy are produced, a flash or an explosion occurs. The nuclear reactions are unstable in degenerate matter. This process is responsible for the explosion of Type Ia supernovae. It triggers also what is called the helium flash at the tip of the red giant branch (RGB) for stars with masses below about $1.8 M_{\odot}$ (at solar metallicity).

\subsubsection{The five mass ranges}

The different behaviours of both contraction and nuclear reaction rates in perfect gas and degenerate regimes are the main causes for the variety of evolution paths followed by stars of different initial masses. Schematically five mass ranges can be considered (Maeder 2009).

(1) Substellar objects. This range corresponds to objects that during their contraction phase enter the degenerate regime before hydrogen ignition. Further contraction cools the central regions as explained above, and thus these central regions never reach the appropriate conditions for hydrogen ignition. These objects become the so-called brown dwarfs. According to Baraffe (2002) the stellar/substellar transition occurs for a mass around $0.075 M_{\odot}$. Note that in objects more massive than $0.012 M_{\odot}$ (Chabrier et al. 2000; Saumon et al. 1996) deuterium burning occurs.

(2) The very low-mass stars. This range corresponds to stars which go only through the hydrogen-burning phase (progenitors of helium white dwarfs). These stars have initial masses between 0.075 and $0.5 M_{\odot}$. Note that single stars in this mass range have main-sequence lifetimes greater than the age of the Universe. Thus the observed helium white dwarfs probably originate from more massive progenitors in close binary systems (Benvenuto \& De Vito 2004) and not from single low-mass stars.

(3) The low-mass stars. Stars with masses between 0.5 and $1.7-2.5 M_{\odot}$ evolve only through the hydrogen- and helium-burning phase (progenitors of carbonoxygen white dwarfs). Helium ignition occurs in degenerate conditions. This 
produces what is called a helium flash. This process is not destructive for the star. It releases in a short time sufficient energy to remove degeneracy in the central regions. After the helium-flash, core helium burning continues smoothly in nondegenerate conditions until the production of a carbon-oxygen white dwarf in the central region. The envelope is ejected by mass loss occurring along the red giant and asymptotic giant branches.

(4) The intermediate-mass stars. Stars in this range ignite helium in non-degenerate conditions (no helium flash). Their end stellar state is either carbon-oxygen white dwarfs (i.e. same as the low-mass stars) or oxygen-neon white dwarfs, if carbon burning can occur in the core. They have initial masses between 0.5 and about $8 M_{\odot}$. The upper limit is still quite difficult to obtain from models due to uncertainties in the sizes of the convective cores and mass loss (Siess 2007). There may be some other possible final fates such as the complete disruption of the star due to carbon burning in degenerate conditions or production of a neutron star by collapse of the core before neon ignition due to electron captures (Maeder 2009). The value of $8 M_{\odot}$ quoted here is obtained from observations of white dwarfs in stellar clusters which allow one to deduce the initial masses of the stars having given birth to the white dwarfs. Relations between the mass of the white dwarf and the initial mass of the progenitor can be derived, in particular the initial mass of stars giving birth to white dwarfs just below the Chandrasekhar mass. Let us note that this method is not free from any theoretical input since the deduction of the initial mass of the white dwarf progenitors is deduced from stellar models (Kalirai et al. 2008)!

(5) Massive stars. This range corresponds to all stars that can evolve beyond the carbon-burning phase until the formation of an iron core (progenitors of neutron stars and black holes), or until the pair instability is encountered (in general during oxygen burning) and the star explodes (Heger et al. 2003). Generally massive stars have initial masses greater than about $8 M_{\odot}$ (Maeder \& Meynet 1989). These stars are the main source of oxygen $\left({ }^{16} \mathrm{O}\right)$ in the Universe.

Most of this oxygen is produced during the core He-burning phase (non-explosive nucleosynthesis), and in general the explosive nucleosynthesis does not affect significantly the yields of oxygen isotopes (see Sect. 3.2.15).

Classical novae occur in binary systems consisting of a carbon-oxygen or oxygenneon-magnesium white dwarf accreting hydrogen-rich matter from a main-sequence companion. Sporadically, a thermonuclear runaway leads to the synthesis of new isotopes and to the ejection of material from the surface of the accreting white dwarf. Concerning the oxygen isotopes, hydrodynamical models of novae show that these objects do not contribute to the synthesis of ${ }^{16} \mathrm{O}$ and ${ }^{18} \mathrm{O}$, but could contribute in making ${ }^{17} \mathrm{O}$ (José et al. 1998; Starrfield et al. 2009, 1974). 
Table 3.12. Model characteristics. The star ages in Myr at the end of the first three nuclear phases are indicated. For the last three, the duration of the considered phase is indicated in years. The third column gives the actual mass of the star at the considered evolutionary stage. The central temperature and density are given in columns 4 and 5. The quantities $\mathrm{q}_{c c}$ and $\mathrm{q}_{e n v}$ correspond to the fraction of the mass encompassed respectively in the convective core and in the convective envelope. The last two columns indicate the ratios of nitrogen to carbon and of nitrogen to oxygen at the surface (ratios of mass fractions).

\begin{tabular}{lcccccccc}
\hline Phase & $\begin{array}{c}\text { Age } \\
{[\mathrm{Myr}]}\end{array}$ & $\begin{array}{c}\text { Mass } \\
M_{\odot}\end{array}$ & $\log \mathrm{T}_{c}$ & $\log \rho_{c}$ & $\mathrm{q}_{c c}$ & $\mathrm{q}_{e n v}$ & $\mathrm{~N} / \mathrm{C}$ & $\mathrm{N} / \mathrm{O}$ \\
\hline & & & & & & & & \\
End H & 6.60 & 24.20 & 7.875 & 1.518 & 0.090 & 0.000 & 0.31 & 0.11 \\
End He & 7.30 & 16.17 & 8.539 & 3.554 & 0.013 & 0.000 & 16.2 & 2.21 \\
End C & 7.32 & 16.00 & 9.127 & 6.563 & 0.000 & 0.464 & 19.1 & 2.38 \\
End Ne & $+50.4 \mathrm{y}$ & 16.00 & 9.218 & 6.594 & 0.000 & 0.464 & 19.1 & 2.38 \\
End O & $+0.3 \mathrm{y}$ & 16.00 & 9.411 & 7.374 & 0.000 & 0.166 & 19.1 & 2.38 \\
End Si & $+0.1 \mathrm{y}$ & 16.00 & 9.787 & 8.638 & 0.001 & 0.062 & 19.1 & 2.38 \\
\hline
\end{tabular}

\subsubsection{Massive stars}

\subsubsection{A reference case: a $25 M_{\odot}$ star}

We have plotted in Figures 3.15 to 3.17 the chemical structure of a $25 M_{\odot}$ star at various stages during its evolution (Hirschi et al. 2004). The metallicity is $Z=0.02$ and the effects of rotation are not included. Several model characteristics are presented in Table 3.12 .

\section{Abundance profiles at the end of the main sequence}

During the main-sequence phase, the abundance of ${ }^{16} \mathrm{O}$ is decreased in the central region as a consequence of the $\mathrm{CNO}$ cycle. At the end of the core H-burning phase, its abundance in the central region is about a factor of 30 lower than the initial value (see top panel of Fig. 3.15). In the outer part of the star (for $M_{r} / M_{\odot}>15$ ), its abundance has not been changed. In the zone comprised between $\sim 8 M_{\odot}$ and $15 M_{\odot}$, the abundance of oxygen increases in steps from the central value to that in the envelope. These steps result from various intermediate convective zones which appear above the core during the main-sequence phase. The situation for ${ }^{17} \mathrm{O}$ is somewhat different. In the same manner as ${ }^{16} \mathrm{O},{ }^{17} \mathrm{O}$ is destroyed in the central region and has kept its initial abundance in the envelope. However it is produced in the region comprised between 11 and $15 M_{\odot}$. This arises from the fact that in the CNO cycle, this isotope is produced at temperatures below about 25 million degrees while it is destroyed at higher temperatures; hence its maximum abundance in regions of partial $\mathrm{H}$ burning. The last stable isotope of oxygen ${ }^{18} \mathrm{O}$ 

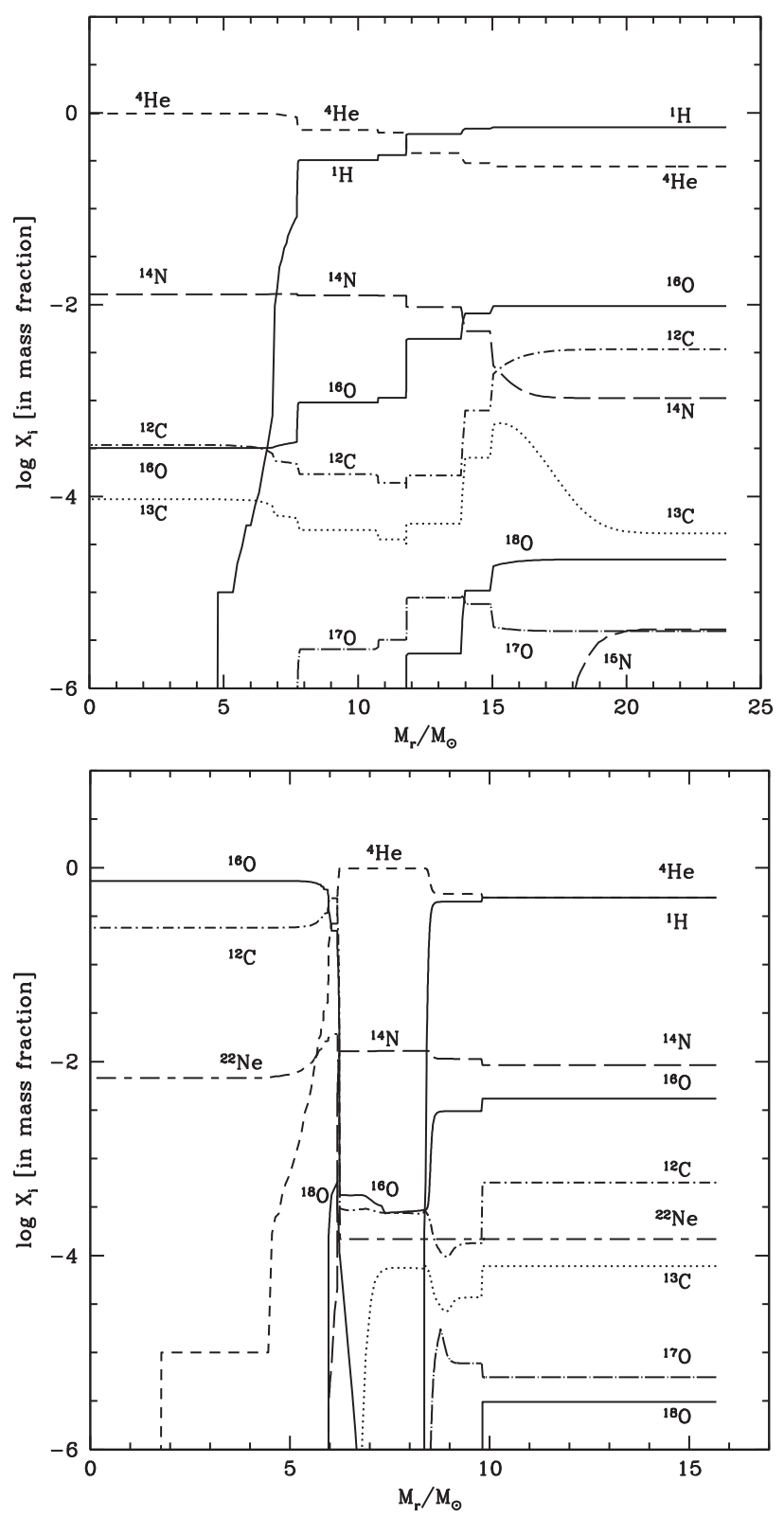

Fig. 3.15. Variations of the abundances (logarithm of the mass fraction) of some elements as a function of the mass (in solar units) inside a $Z=0.02$ stellar model with an initial mass of $25 M_{\odot}$. Top: at the end of the core H-burning phase. At this stage, the surface abundances have kept their initial values. Bottom: same as top figure but at the end of the core He-burning phase. The total mass is now $16 M_{\odot}$ because of the effect of stellar winds. 

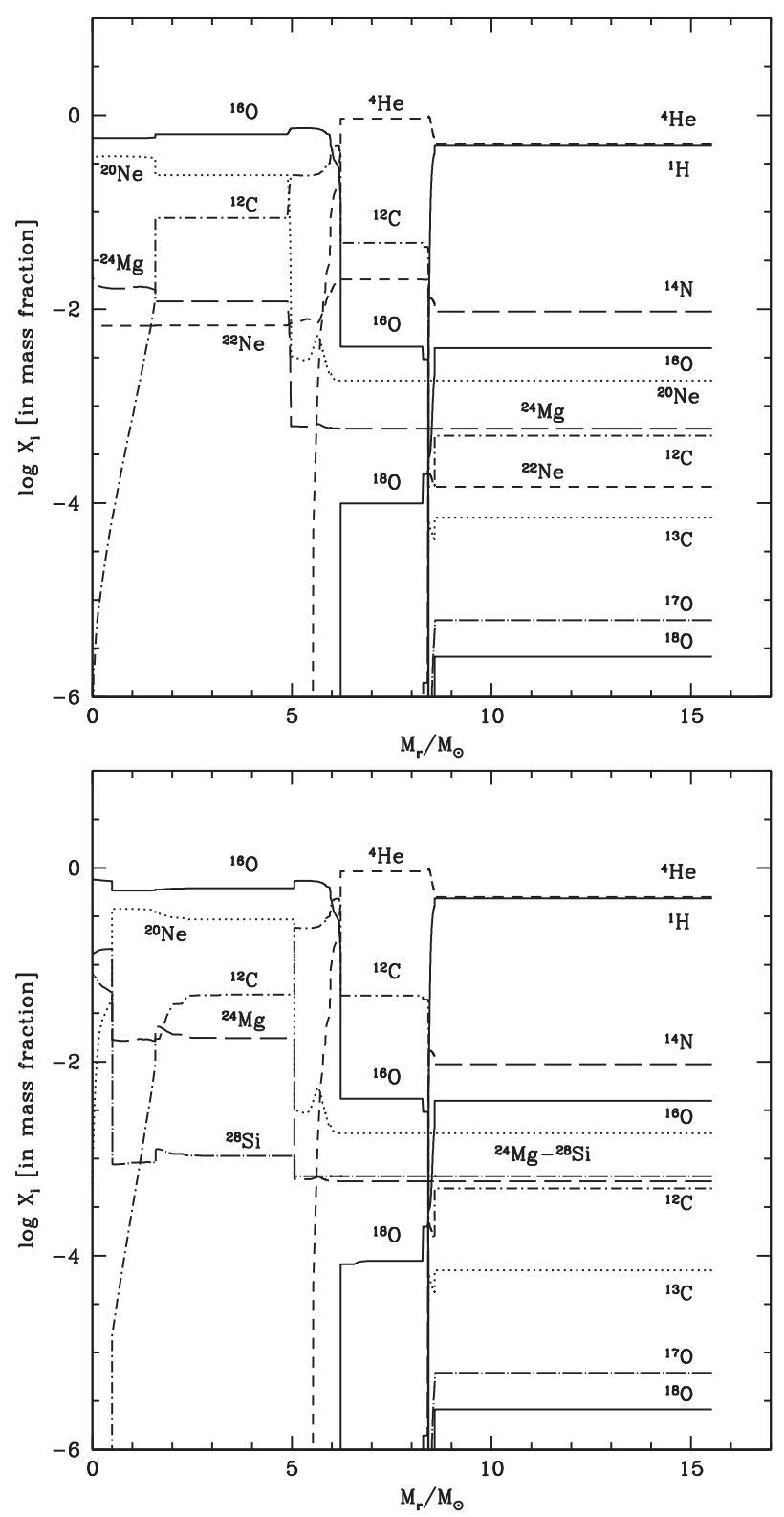

Fig. 3.16. Top: same as in Figure 3.15 at the end of the core C-burning phase. Bottom: same as in Figure 3.15 at the end of the core Ne-photodisintegration phase.

is completely destroyed in the central regions. As for ${ }^{16} \mathrm{O}$ and in contrast to ${ }^{17} \mathrm{O}$, there is no region in the star where this element has been produced at this stage of the evolution. 

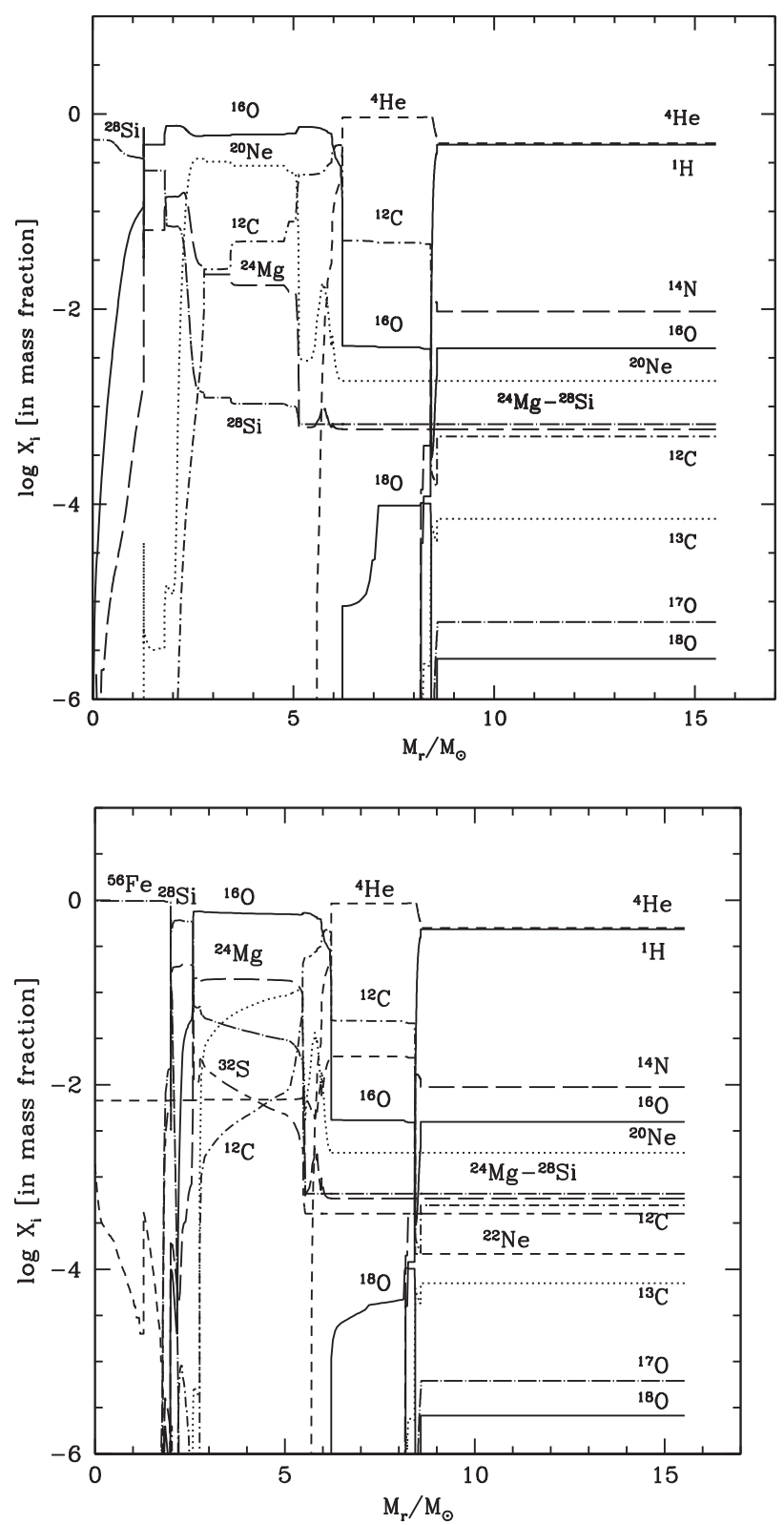

Fig. 3.17. Top: same as in Figure 3.15 at the end of the core O-burning phase. Bottom: same as in Figure 3.15 at the end of the core Si-burning phase.

Thus at the end of the main sequence phase, the ${ }^{16} \mathrm{O}$ content of the star has decreased. Initially the integrated mass of ${ }^{16} \mathrm{O}$ in the star was about $0.24 M_{\odot}$. 
At the end of the main sequence phase, it is equal to $0.11 M_{\odot}$. A very small part, $0.008 M_{\odot}$, has been lost by stellar wind. Most of the decrease of the ${ }^{16} \mathrm{O}$ abundance is due to its transformation into ${ }^{14} \mathrm{~N}$ through the CNO cycle. The integrated mass of ${ }^{17} \mathrm{O}$ has also decreased. The decrease is however much weaker than for ${ }^{16} \mathrm{O}$ going down from $10^{-4}$ to $8 \times 10^{-5} M_{\odot}$. The integrated mass of ${ }^{18} \mathrm{O}$ has decreased from $5.5 \times 10^{-4}$ to $2.2 \times 10^{-4} M_{\odot}$.

\section{Abundance profiles at the end of the core He-burning phase}

The core He-burning phase is the main phase of ${ }^{16} \mathrm{O}$ production.

The chemical structure of the star at the end of the core He-burning phase is shown in the bottom panel of Figure 3.15. The core He-burning phase has a lifetime of $0.7 \mathrm{Myr}$. This corresponds to $10.6 \%$ of the core H-burning phase lifetime. In the central region, helium has been transformed into ${ }^{12} \mathrm{C}$ and ${ }^{16} \mathrm{O}$ through the $3 \alpha$ reaction followed by ${ }^{12} \mathrm{C}(\alpha, \gamma){ }^{16} \mathrm{O}$. Outside the core, the abundance of ${ }^{16} \mathrm{O}$ is still inferior to its initial value. In the H-burning shell, this is of course a consequence of the CNO cycle. Two zones can be recognised in this region. In the inner one, which extends from 6 to $8.3 M_{\odot}$, complete $\mathrm{H}$ burning occurs. In the outer one, which extends from 8.3 to $9.7 M_{\odot}, \mathrm{H}$ burning is only partial and a much higher ${ }^{16} \mathrm{O}$ abundance is obtained. Outside the H-burning shell, there is a decrease of the ${ }^{16} \mathrm{O}$ abundance by more than a factor of 2 with respect to the initial value due to dredge-up. The ${ }^{17} \mathrm{O}$ isotope is completely destroyed in the core. It is also destroyed in the inner H-burning shell and produced in small amounts in the outer $\mathrm{H}$-burning shell. At the surface, the abundance of ${ }^{17} \mathrm{O}$ has increased from 4 to $6 \times 10^{-6}$ due to dredge-up. At the very beginning of the core He-burning phase, the chain of reactions

$$
{ }^{14} \mathrm{~N}(\alpha, \gamma){ }^{18} \mathrm{~F}(\beta, \nu){ }^{18} \mathrm{O}(\alpha, \gamma){ }^{22} \mathrm{Ne}
$$

transforms ${ }^{14} \mathrm{~N}$ into ${ }^{22} \mathrm{Ne}$ via ${ }^{18} \mathrm{O}$. In the nascent He-burning shell, the transformation is not complete producing a steep rise in ${ }^{18} \mathrm{O}$. In the whole region processed by $\mathrm{H}$-burning, ${ }^{18} \mathrm{O}$ is destroyed. At the surface, due to dredge-up, the ${ }^{18} \mathrm{O}$ abundance has decreased from 22 to $3 \times 10^{-6}$.

At the end of this phase, the integrated mass of ${ }^{16} \mathrm{O}$ in the star reaches a value of $4.4 M_{\odot}$. This means that the mass of oxygen has increased by more than a factor 18 with respect to its initial value. For ${ }^{17} \mathrm{O}$ the situation has not drastically changed with respect the one reached at the end of the core H-burning phase, while for ${ }^{18} \mathrm{O}$, despite the peak in the He-burning shell, the integrated mass has decreased.

\section{Abundance profiles at the end of the core C-burning phase}

At the end of the C-burning phase (see top panel of Fig. 3.16), the abundance of ${ }^{16} \mathrm{O}$ in the inner $1.5 M_{\odot}$ of the star has slightly decreased. The core remains radiative during the C-burning phase as a consequence of the high neutrino flux. Indeed the neutrinos are very efficient in evacuating the energy produced in the 
core, and prevent the apparition of a convective zone in the central region. Between 1.5 and $5 M_{\odot}$, one can see the abundances resulting from the activity of the C-burning shell. Again the abundance of ${ }^{16} \mathrm{O}$ is slightly decreased with respect to the value reached at the end of the core He-burning phase. Between 5 and $6 M_{\odot}$, one can see a region where the abundances of ${ }^{16} \mathrm{O}$ and ${ }^{12} \mathrm{C}$ have remained unchanged since the end of the core He-burning phase. The activity of the Heburning reaction extends from 6 to about $8.5 M_{\odot}$. In that region, important changes have taken place: a small quantity of helium has been transformed into ${ }^{12} \mathrm{C}$ and ${ }^{16} \mathrm{O}$, while ${ }^{14} \mathrm{~N}$ has been transformed partly in ${ }^{18} \mathrm{O}$ and partly in ${ }^{22} \mathrm{Ne}$. Between this He-burning zone and the envelope, there is a very thin H-burning shell characterised by a decrease of ${ }^{12} \mathrm{C}$ and ${ }^{16} \mathrm{O}$ abundances and an increase of the ${ }^{14} \mathrm{~N}$ abundance. The abundances in the envelope do not show great changes with respect to the situation reached at the end of the core He-burning phase.

At the end of this phase, the integrated mass of ${ }^{16} \mathrm{O}$ is $3.9 M_{\odot}$, slightly less than at the end of the previous phase. This is mainly due to the destruction of ${ }^{16} \mathrm{O}$ in the central region and, to a lesser extent, to mass loss (see below for more details). The integrated mass of ${ }^{17} \mathrm{O}$ has not changed significantly, while that of ${ }^{18} \mathrm{O}$ has nearly doubled due to nuclear processes in the He-burning shell.

\section{Abundance profiles at the end of the Ne-photodisintegration phase}

The abundances at the end of the Ne-photodisintegration phase are shown in the bottom panel of Figure 3.16. The abundances of all the oxygen isotopes are little affected, and therefore, their integrated masses are not significantly changed with respect to the values obtained at the end of the core C-burning phase.

\section{Abundance profiles at the end of the core O-burning phase}

The abundance profiles at the end of the core O-burning phase are shown in the top panel of Figure 3.17. Core O-burning has depleted oxygen in the inner $1 M_{\odot}$. Between 1 and $5 M_{\odot}$, the abundance profiles result from various shell burnings. Going from the centre outwards (inside out), results from the nuclear activity of the O-, Ne- and C-burning shells can be seen. Beyond $5 M_{\odot}$, no significant changes have occurred. The only noticeable ones are the abundance decreases of ${ }^{18} \mathrm{O}$ and ${ }^{17} \mathrm{O}$ between 6.2 and $7 M_{\odot}$. This is due to the increased temperature in this region when the central parts of the star contract.

As a result of its destruction in the core, the integrated mass of ${ }^{16} \mathrm{O}$ has been lowered from 3.9 to $3.1 M_{\odot}$. The mass of ${ }^{17} \mathrm{O}$ has not changed significantly, while that of ${ }^{18} \mathrm{O}$ continues to decrease.

\section{Abundance profiles at the end of the core Si-burning phase}

They are shown in the bottom panel of Figure 3.17. ${ }^{16} \mathrm{O}$ has now been completely destroyed in the inner 2.5 $M_{\odot}$. Beyond these $2.5 M_{\odot}$, the abundances of all the oxygen isotopes are unchanged. 

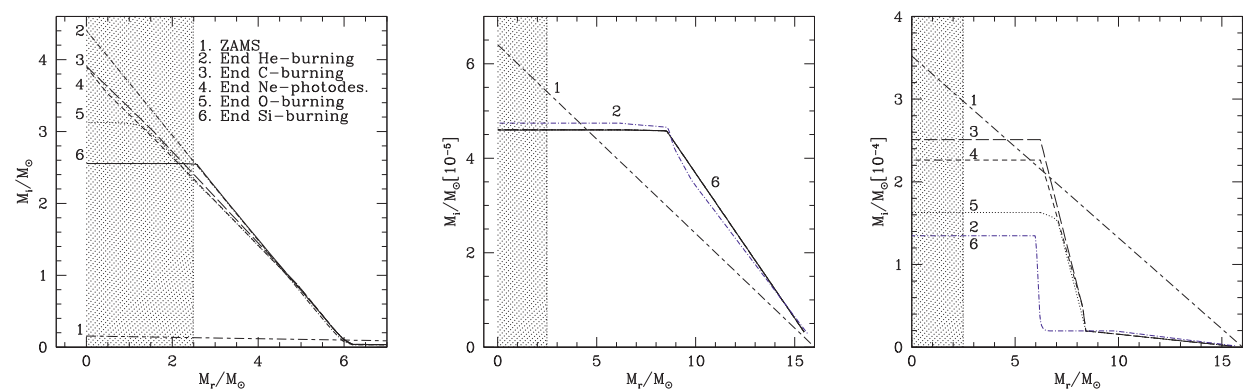

Fig. 3.18. Left: ${ }^{16} \mathrm{O}$ mass ejected at a specific evolutionary stage if the whole mass above $M_{r}$ were ejected. The ordinate corresponds to the integration of the mass of ${ }^{16} \mathrm{O}$ performed from the value of $M_{r}$ indicated on the abscissas up to the surface. This means that the ordinate corresponding to the abscissa 0 is equal to the total quantity of ${ }^{16} \mathrm{O}$ inside the star at the corresponding stage. Note that for all the models considered here the actual total mass of the star does not change much and is around $16 M_{\odot}$ (see text). This implies that the variations of the integrated quantities are due to nuclear processes occurring in the star and not to loss of mass through the effect of stellar winds. The inner $2.5 M_{\odot}$ are hatched to indicate that this part of the star will probably remain locked in a compact remnant (neutron star or black hole). Middle: same as left panel for ${ }^{17} \mathrm{O}$. Note that curves 3, 4 and 5 are identical to curve 6 . Right: same as left panel for ${ }^{18} \mathrm{O}$.

At this stage the total mass of ${ }^{16} \mathrm{O}$ in the star is $2.6 M_{\odot}$, and that of ${ }^{17} \mathrm{O}$ and of ${ }^{18} \mathrm{O}$ are respectively of $46 \times 10^{-6}$ and $12 \times 10^{-5} M_{\odot}$.

\section{So, when are the oxygen isotopes produced?}

In the left panel of Figure 3.18 we show the total quantity of ${ }^{16} \mathrm{O}$ which would be ejected if, at a specific evolutionary stage, the whole mass above $M_{r}$ were ejected. For instance, if at the end of the core helium-burning phase the whole envelope above $2.5 M_{\odot}$ were ejected, then a total quantity of about $2.6 M_{\odot}$ of oxygen would be ejected. In other words the quantity plotted on the vertical axis is obtained using the expression

$$
M_{i}\left(M_{r}\right)=\int_{M_{r}}^{M} X_{i}\left(m_{r}\right) \mathrm{d} m_{r}
$$

with $M$ being the actual star mass at the evolutionary stage under consideration and $X_{i}\left(m_{r}\right)$ the oxygen mass fraction in the model at that stage and at the Lagrangian mass coordinate, $m_{r}$. Note that the curve labelled ZAMS is obtained simply by using for $X_{i}\left(m_{r}\right)$ a constant value equal to the initial abundance of the considered isotope. We performed the integration only over the central $16 M_{\odot}$ of our $25 M_{\odot}$ model. The value of $16 M_{\odot}$ corresponds to the final mass of the star at the pre-supernova stage. This mass results from the effects of stellar winds occurring during the whole stellar lifetime. Restricting the integration over $16 M_{\odot}$ 
allows the comparison of quantities integrated over the same part of the star at all the evolutionary stages.

Two interesting features are brought to light from such a plot: first, it shows the effect of the different nuclear phases on the integrated masses of ${ }^{16} \mathrm{O}$; second, it allows one to see how the ejected mass would change for different mass cuts (in that case curve number 6 corresponding to the pre-supernova stage must be considered).

Looking at the left panel of Figure 3.18, a few interesting points may be underlined:

- The mass of ${ }^{16} \mathrm{O}$ in the layers outside the hatched zone, i.e. outside the zone which may remain locked in the stellar remnant, does not change much after the end of the core C-burning stage. Even after the core He-burning phase, the mass of ${ }^{16} \mathrm{O}$ in this region is almost the one obtained at the pre-supernova stage.

- One notes that the region which will contribute to the ${ }^{16} \mathrm{O}$ production is comprised between 2.5 and $6 M_{\odot}$.

- If the cutoff between the remnant and ejecta masses were smaller than the mass considered here $\left(2.5 M_{\odot}\right)$, the ${ }^{16} \mathrm{O}$ yields would be little affected. This is because in these inner regions ${ }^{16} \mathrm{O}$ is mostly destroyed.

The integrated masses of ${ }^{17} \mathrm{O}$ and ${ }^{18} \mathrm{O}$ are shown in the middle and right panels of Figure 3.18. We can note the following points.

- At the end of the core He-burning phase, the star already has the ${ }^{17} \mathrm{O}$ mass which will be present at the pre-supernova stage. This is not the case for ${ }^{18} \mathrm{O}$.

- ${ }^{17} \mathrm{O}$ is produced in the $\mathrm{H}$-burning zones (outside $M_{r}=8.5 M_{\odot}$ ).

- ${ }^{18} \mathrm{O}$ is created in the partial He-burning regions. A slight excess of this isotope is observed in the region around $6 M_{\odot}$ at the end of core C-burning phase, but this excess of ${ }^{18} \mathrm{O}$ is then destroyed during the subsequent evolutionary stages.

- At the pre-supernova stage, the masses for both isotopes are smaller than those initially present.

Before discussing how the present results vary as a function of initial mass, metallicity and rotation, and how other physical ingredients may also impact them, let us just say a few words about the effect of mass loss on this particular model. As can be seen from Table 3.12, most of the mass loss occurs during the core He-burning stage (loss of $8 M_{\odot}$ ). During that stage, due to dredge-up, matter 

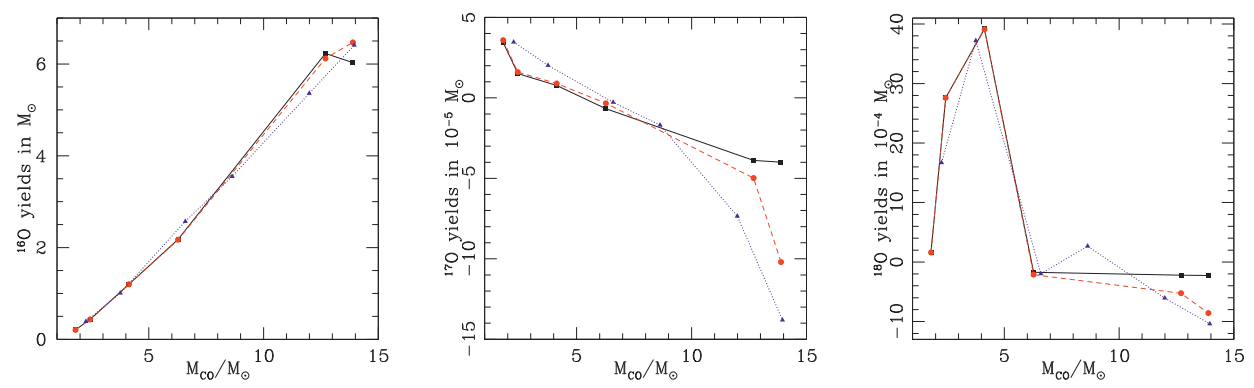

Fig. 3.19. Left: the solid lines shows the yields of ${ }^{16} \mathrm{O}$ in the ejecta of the supernova as a function of the CO core mass (models from Hirschi et al. 2004). The initial masses corresponding to each dot from left to right are 12, 15, 20, 25, 40 and $60 M_{\odot}$ respectively (solar metallicity, non-rotating models). The dashed line shows the complete yields, i.e. the yields of the supernova and of the stellar winds. The dotted line shows the complete yields for rotating models with $v_{\text {ini }}=300 \mathrm{~km} \mathrm{~s}^{-1}$. Middle: same as left panel for ${ }^{17} \mathrm{O}$. Right: same as left panel for ${ }^{18} \mathrm{O}$.

is depleted in ${ }^{16} \mathrm{O}$ and ${ }^{18} \mathrm{O}$ but slightly enriched in ${ }^{17} \mathrm{O}$. For ${ }^{16} \mathrm{O}$ the contribution of the wind is completely negligible. Indeed the wind yield ${ }^{3}$ for this isotope, namely $-1.07 \times 10^{-2} M_{\odot}$, has an absolute value which amounts to only $5 \times 10^{-3}$ of the yield in the supernova ejecta $\left(2.18 M_{\odot}\right)$. For the other two isotopes, wind and supernova yields are of the same order $\left({ }^{17} \mathrm{O}\right.$ : wind $+3.4 \times 10^{-6}$, supernova $-6.7 \times 10^{-6} ;{ }^{18} \mathrm{O}$ : wind $-3.88 \times 10^{-5}$, supernova $-17.4 \times 10^{-5}$ in solar masses). Thus in that case winds do affect the final total yield (wind and supernova), but of course it remains to be seen to which extent a $25 M_{\odot}$ star contributes to the destruction or production of these two isotopes. As we shall see this is probably not the case.

\subsubsection{Effects of mass, metallicity, rotation, etc.}

Mass. How does the situation change when the initial mass varies? For masses lower than $25 M_{\odot}$, at standard metallicity $(Z=0.02)$, the main thing affecting the ${ }^{16} \mathrm{O}$ yields is the mass of the $\mathrm{CO}$ core which decreases when the initial mass decreases. In the left panel of Figure 3.19, we show the ${ }^{16} \mathrm{O}$ yields as a function of the mass of the $\mathrm{CO}$ core at the pre-supernova stage ${ }^{4}$. We see that at least for $M \leq 25 M_{\odot}$ the relation is not far from linear. For the larger masses, the situation is less clear due to the action of the stellar winds which may peel off the CO core,

\footnotetext{
${ }^{3}$ The yield of an element is the difference between its mass in the ejecta at the time of the ejection and its mass initially present in the same portion of the star. Yields may be negative when the element is destroyed by the star.

${ }^{4}$ Note that the mass of the CO core at the end of the core C-burning stage is already very close to the value obtained at the pre-supernova stage.
} 
and thus decrease the quantity of ${ }^{16} \mathrm{O}$ at the time of the pre-supernova (see below for a more detailed discussion of this point).

The ${ }^{17} \mathrm{O}$ yield decreases when the initial mass increases, becoming even negative for stars with $M \geq 25 M_{\odot}$ as can be seen in the middle panel of Figure 3.19. This reflects the fact that ${ }^{17} \mathrm{O}$ is produced in H-burning regions with temperatures lower than a given limit. For ${ }^{18} \mathrm{O}$ (see the right panel of Fig. 3.19) the situation is more complicated in the sense that the yield of this isotope first increases with mass until a maximum is reached around $20 M_{\odot}$, then it decreases becoming negative for stellar models with higher initial mass. This behaviour results from the fact that ${ }^{18} \mathrm{O}$ is what we might tentatively call a "transitory element" in a chain of reactions. This means that its peak abundance will be reached for a relatively narrow domain of temperatures. Indeed the temperatures have to be high enough to allow the chain to synthesise it but low enough to prevent its destruction.

It is interesting to compare the yields of ${ }^{17} \mathrm{O}$ and ${ }^{18} \mathrm{O}$ in massive stars with those obtained by Marigo (2001) for intermediate mass stars. Marigo (2001) shows that ${ }^{17} \mathrm{O}$ may be produced in intermediate mass stars with initial masses above about $4 M_{\odot}$ with yields of the order of a few $10^{-3} M_{\odot}$. This is well above what the massive stars in the range between 12 and $20 M_{\odot}$ can do. Above $20 M_{\odot},{ }^{17} \mathrm{O}$ is destroyed but at a level which probably remains negligible with respect to what is produced in intermediate mass stars. Thus for ${ }^{17} \mathrm{O}$ intermediate mass stars appear as a much more promising source than massive stars.

The contrary occurs for ${ }^{18} \mathrm{O}$. Indeed, according to Marigo (2001), this element is destroyed in intermediate mass stars with initial masses above about $4 M_{\odot}$. As can be seen in the right panel of Figure 3.19, it is produced in the 15 and $20 M_{\odot}$ models.

Metallicity. When one discusses the effects of a change of the initial metallicity on the yields of a given element, it is useful to first discern if the isotope considered is a primary or a secondary element. By primary element we mean an element which would be produced in about the same quantity in a Population III star as in a Population I star, i.e. an element whose yield does not depend much on the initial metallicity of the star. Secondary elements, on the contrary, are those whose yields crucially depend on the initial abundance in heavy elements of the star. Their yields are thus very different in Population III and Population I stars. It is well known that ${ }^{16} \mathrm{O}$ is in that respect a primary element. This comes from the fact that it is produced from helium in the core helium-burning phase. Whatever the initial metallicity the core, it becomes almost pure helium by the end of the core H-burning phase. Of course there is still a metallicity dependence due to the fact that the physical conditions, such as temperature and density, are different depending on the initial metallicity of the star, but these effects are of second order importance. The production of the other two oxygen isotopes depends on the initial CNO content of the star. For instance ${ }^{18} \mathrm{O}$ results from the transformation of the ${ }^{14} \mathrm{~N}$ produced during the core H-burning phase, whose abundance is equivalent to the initial value of the sum of the CNO elements. However since their production occurs only for relatively narrow physical 
Table 3.13. Yield comparisons for $20 M_{\odot}$ non-rotating and rotating stellar models at various metallicities. The yields are in solar masses. Data from Hirschi (2007).

\begin{tabular}{l|ccc|ccc}
\hline Initial Z & $\mathrm{Y}\left({ }^{16} \mathrm{O}\right)$ & $\begin{array}{r}\mathrm{Y}\left({ }^{17} \mathrm{O}\right) \\
v=0\end{array}$ & $\mathrm{Y}\left({ }^{18} \mathrm{O}\right)$ & $\mathrm{Y}\left({ }^{16} \mathrm{O}\right)$ & $\begin{array}{c}\mathrm{Y}\left({ }^{17} \mathrm{O}\right) \\
v=300 \mathrm{~km} \mathrm{~s}^{-1}\end{array}$ & $\begin{array}{c}\mathrm{Y}\left({ }^{18} \mathrm{O}\right) \\
\end{array}$ \\
\hline $10^{-3}$ & 1.46 & $-5.5 \mathrm{e}-7$ & $-1.1 \mathrm{e}-5$ & 2.70 & $4.85 \mathrm{e}-7$ & $-1.89 \mathrm{e}-5$ \\
$10^{-5}$ & 1.50 & $3.1 \mathrm{e}-7$ & $-9.4 \mathrm{e}-8$ & 2.37 & $3.40 \mathrm{e}-7$ & $5.27 \mathrm{e}-7$ \\
$10^{-8}$ & 1.20 & $1.9 \mathrm{e}-7$ & $-2.2 \mathrm{e}-10$ & 1.96 & $1.70 \mathrm{e}-8$ & $2.14 \mathrm{e}-7$ \\
\hline
\end{tabular}

conditions, their yields in the global ejecta may not present any clear trend with metallicity. This is what occurs in massive stars.

Let us compare the yields of three non-rotating $20 M_{\odot}$ models computed with the same physical ingredients except for different initial abundances. The yields are shown in Table 3.13. The yields of ${ }^{16} \mathrm{O}$ are almost constant, while those of ${ }^{17} \mathrm{O}$ and ${ }^{18} \mathrm{O}$ vary significantly. Moreover, no clear trend with metallicity is noticed thus illustrating the point discussed above.

Overshooting. The size of the convective cores is poorly known from $a b$ initio physical principles. This is a very important quantity since it affects the lifetime and the nucleosynthesis of a given star. In stellar models, the limit of the convective core is determined through criteria which may vary from one author to another. A typical approach to compute the size of the convective core is to determine the point within the star where the convective cells have zero velocity. Overshooting increases the size of the convective cores, and as a result, a star of a given initial mass with overshooting will have a behaviour roughly similar to that of a star of higher initial mass without it. It is difficult to be more quantitative here since, in order to study this effect, a series of models with exactly the same physical ingredients should be computed for different values of the overshooting parameter. Let us just give an example: the ejected mass of ${ }^{16} \mathrm{O}$ in a $20 M_{\odot}$ stellar model without overshooting is $2.0 M_{\odot}$ while it is $3.1 M_{\odot}$ in the same model when a moderate overshooting (of $0.25 \mathrm{H}_{p}$ ) is taken into account (Meynet 1990). Thus the effect is quite important.

The reaction ${ }^{12} \mathbf{C}(\alpha, \gamma){ }^{16} \mathrm{O}$. In the list of important nuclear reaction rates in astrophysics, ${ }^{12} \mathrm{C}(\alpha, \gamma){ }^{16} \mathrm{O}$ appears in the top rank as reflected by the abundant literature on the subject. It affects not only the quantities of carbon and oxygen synthesised by the star, but has an impact on many other stellar model outputs, e.g. the formation of blue loop during the core helium-burning phase of massive stars or the nature of the stellar remnant at the end of the evolution (see for instance the text by Woosley in Hauck et al. 1986). There is still some uncertainty pertaining to this yield which amounts to about a factor of 2. Recently, Tur et al. (2007) have studied the impact of a rate change for this reaction on the production of the ${ }^{16} \mathrm{O}$ and ${ }^{18} \mathrm{O}$ isotopes (see their Fig. 3). 
Mass loss. Mass loss intensity is a key quantity in stellar evolution. It affects the tracks in the HR diagram, lifetimes, surface abundances, chemical yields, presupernova status, the nature of the remnant, the mechanical energy released in the interstellar medium, the hardness of the radiation field, etc.

The main mass-loss trigger is radiation pressure. Quoting Eddington (1926), "the radiation observed to be emitted must work its way through the star, and if there were too much obstruction it would blow up the star." Note that although it was already realised in the 1920s that radiation pressure might produce mass loss, it is only in the late 1960s, when sensitive UV diagnostics of mass loss from O stars became available, that the effects of mass loss on the evolution of stars were really considered.

Radiation triggers mass loss through the line opacities in hot stars. It may also power strong mass loss through the continuum opacity when the star is near the Eddington limit. For cool stars, radiation pressure is exerted on dust grains. Note that other mechanisms such as pulsations, mass transfer in close binary systems or rotation near the critical limit may also trigger mass loss episodes.

For hot stars, typical values for the terminal wind velocity $v_{\infty}$ are of the order of 3 times the escape velocity, i.e. about $2000-3000 \mathrm{~km} \mathrm{~s}^{-1}$, and mass loss rates are between $10^{-8}-10^{-4} M_{\odot}$ per year. Luminous Blue Variable (LBV) stars during outbursts show mass loss rates as high as $10^{-4}-10^{-1} M_{\odot}$ per year (Lamers \& Cassinelli 1999).

According to recent estimates, the mass loss rate $\dot{M}$ varies with luminosity $L$ as

$$
\dot{M} \sim L^{1.7}
$$

With the mass-luminosity relation for massive stars

$$
L \sim M^{2}
$$

this gives

$$
\dot{M} \sim M^{3.4}
$$

For massive stars, the main-sequence lifetime, $t_{\mathrm{MS}}$, scales as

$$
t_{\mathrm{MS}} \sim M^{-0.6}
$$

the amount $\Delta M$ of mass lost during the main sequence is then

$$
\Delta M \sim M^{2.8}
$$

and the relative mass lost is

$$
\frac{\Delta M}{M} \sim M^{1.8}
$$

Thus, not only the total amount of mass lost grows strongly with the stellar mass, but also the relative amount, which illustrates the importance of this effect. 
In addition to the intensity of the stellar winds for different evolutionary phases, one needs to know how the winds vary with the metallicity. This is a key effect in order to understand the massive-star populations observed in regions of different metallicities. It has also an important impact on the yields expected from stellar models at various metallicities.

Current wisdom considers that very metal-poor stars lose only very small amounts of mass through radiatively driven stellar winds. This comes from the fact that, when the metallicity is low, the number of strong absorbing lines is small, and thus the coupling between the radiative forces and matter is weak. Wind models impose a scaling relation of the type

$$
\dot{M}(Z)=\left(\frac{Z}{Z_{\odot}}\right)^{\alpha} \dot{M}\left(Z_{\odot}\right),
$$

where $\dot{M}(Z)$ is the mass loss rate when the metallicity is equal to $Z$ and $\dot{M}\left(Z_{\odot}\right)$ is the mass loss rate for the solar metallicity, $Z$ being the mass fraction of heavy elements. In the metallicity range from $1 / 30$ to 3.0 times solar, the value of $\alpha$ is between 0.5 and 0.8 according to stellar wind models (Kudritzki et al. 1987; Leitherer et al. 1992; Vink et al. 2001). Such a scaling law implies for instance that a non-rotating $60 M_{\odot}$ star with $Z=0.02$ ends its stellar life with a final mass of $14.6 M_{\odot}$, while the same model with a metallicity of $Z=10^{-5}$ ends its life with a mass of $59.57 M_{\odot}$ ( $c f$. models of Meynet \& Maeder 2005 and Meynet et al. 2006 with $\alpha=0.5)$.

Thus the $60 M_{\odot}$ star with $Z=10^{-5}$ will be expected to give birth to a black hole. In that case nearly all (if not all) the stellar mass may disappear in the remnant preventing the star from enriching the interstellar medium with new synthesised elements (see however the case of Nova Scorpii, González Hernández et al. 2009 in Sect. 3.2.2.4). The metal-rich model, on the other hand, will probably leave a neutron star, and contribute to the enrichment of the interstellar medium through both the winds and the supernova ejecta.

A star that loses a lot of material through stellar winds may enrich the interstellar medium in a different manner to a star that retains most of its mass until the supernova explosion. As Maeder (1992) pointed out, when the stellar winds are strong, material partially processed by the nuclear reactions is released, favouring some species (e.g. helium and carbon which would be partially destroyed if locked in the star) and disfavouring others (e.g. oxygen which would be produced by further transformation of the species which are wind-ejected). Thus the effect of mass loss on ${ }^{16} \mathrm{O}$ are clear: an increase of mass loss will tend to reduce the ${ }^{16} \mathrm{O}$ yield. As a numerical example we can compare the ${ }^{16} \mathrm{O}$ yields obtained from massive star models computed by Maeder (1992) with normal and twice the normal mass loss rate for $Z=0.02$ (see Fig. 3.20). We see that differences are important for stars more massive than $25 M_{\odot}$ : the yields of ${ }^{16} \mathrm{O}$ are multiplied by factors between 2 and 4 for stars in the mass range between 40 and $85 M_{\odot}$ when low mass loss rates are used. In the case of the $120 M_{\odot}$ star, the yield increase is even more spectacular. Let us estimate the effect on the yields for a generation of massive stars. With a Salpeter initial mass function (IMF, see Chapter 4), when $N$ stars 


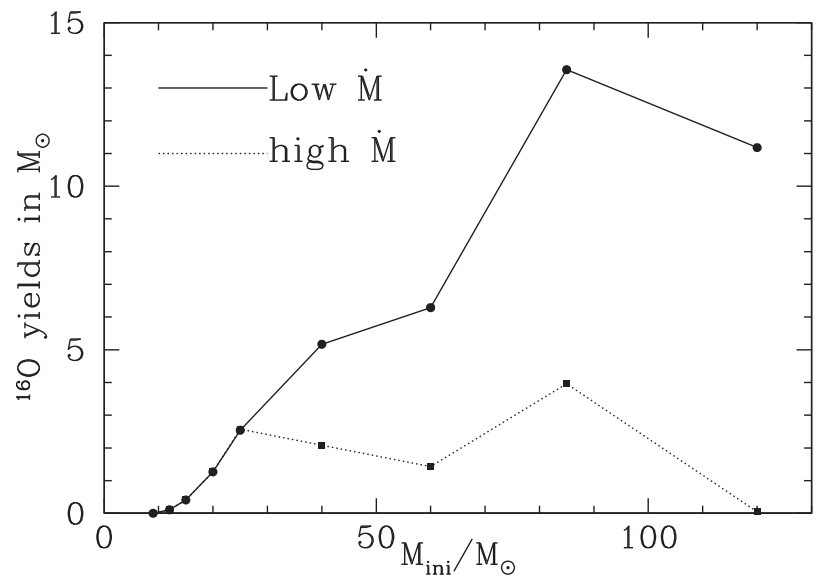

Fig. 3.20. Yields of ${ }^{16} \mathrm{O}$ from the models of Maeder (1992) computed with a high and a low mass loss rate.

are formed there are $0.8 N$ stars with masses between 8 and $25 M_{\odot}$ and $0.2 N$ stars with masses between 25 and $120 M_{\odot}$. Looking at the case with the high mass loss rates in Figure 3.20, we can assume, just for order-of-magnitude estimates, that the yields for stars with $M>25 M_{\odot}$ have the same value $Y_{O}$ for all masses. In the lower mass range let us take a typical yield equal to $Y_{O} / 2$. Thus in the case of high mass loss rate the whole massive star population would release a mass $M_{O}$ of newly synthesised oxygen equal to $\left(0.8 Y_{O} / 2+0.2 Y_{O}\right) N=0.6 Y_{O} N$. When the low mass loss rates are used, the yields of stars between 8 and $25 M_{\odot}$ are not changed, while those of stars with $M>25 M_{\odot}$ are increased. Let us suppose that they are increased by a factor 4 which is clearly an overestimate (except for the $120 M_{\odot}$ case). Then the yield for a whole massive star population would be proportional to $\left(0.8 Y_{O} / 2+4 \times 0.2 Y_{O}\right) N=1.2 Y_{O} N$. Thus, here, lowering the mass loss rates doubles the contribution of massive stars to ${ }^{16} \mathrm{O}$ production. This is of course not negligible (although let us recall that this is clearly an overestimate).

The reduction of the oxygen yield by mass loss will occur at high metallicity where the stellar winds are stronger. Now, since the new element fraction ejected by a stellar generation is the same, the mass which is not ejected in the form of new oxygen must be ejected in the form of another element. Which is this element? The yields of helium, carbon and oxygen are shown in Figure 3.21. Comparing column 1 (very weak mass loss) with column 5 (very strong mass loss), one sees that the deficit of oxygen in the high mass loss regime is more than compensated by a large increase in the yields of helium and carbon.

Rotation. It has been known for a long time that stars rotate. The first known detailed account of the Sun rotation dates back to the time of Galileo Galilei at the beginning of the 17th century. Nowadays our knowledge of the star rotation 


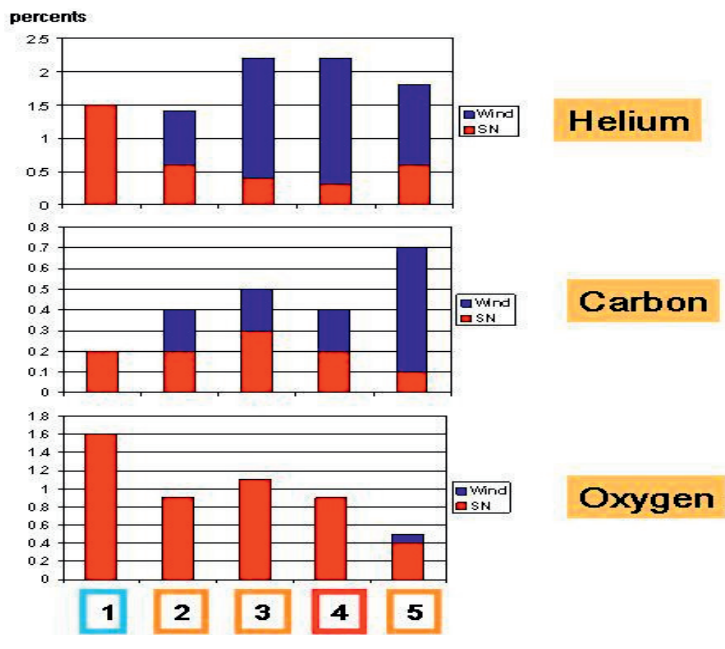

\begin{tabular}{|l|}
\hline $1 \rightarrow Z=0.001$ \\
Schaller et al \\
$(92)$
\end{tabular}

Fig. 3.21. Mass fraction in a generation of stars initially more massive than $8 M_{\odot}$ ejected in the form of new helium, carbon and oxygen. A Salpeter IMF has been used. Various stellar models are compared: the labels MM03 and MM05 are for Meynet \& Maeder (2003) and Meynet \& Maeder (2005), respectively.

is mainly based on Doppler widening of absorption lines. Very recently, interferometric techniques have revealed the shape of some stars strongly deformed by fast rotation as Achernar (Domiciano de Souza et al. 2003; Carciofi et al. 2008) or the variation of the effective temperature with the latitude (Zhao et al. 2009).

Recent works have provided new estimates of the rotation velocity of B-type stars. Dufton et al. (2006) have measured the rotation of stars in two Galactic clusters - NGC 3293 and NGC 4755 - whose ages are comprised between 10 and $15 \mathrm{Myr}$. For stars with masses between 3 and $12 M_{\odot}$, they find that the velocity distribution peaks at $250 \mathrm{~km} \mathrm{~s}^{-1}$ accounting for a random distribution of the inclination ${ }^{5}$. Similar results have been obtained by Huang \& Gies (2006a, $2006 \mathrm{~b})$. These authors also show that the average velocity of stars with masses between 8.5 and $16 M_{\odot}$ remains more or less constant when the surface polar gravity decreases, i.e. when evolution proceeds. On the other hand, for masses between 2.5 and $8.5 M_{\odot}$ the velocity decreases with decreasing polar gravity, indicating that, in this mass range, some breaking mechanism is active which does not work in the higher mass range (magnetic breaking?). Binary systems appear to experience more spin down than single stars.

Huang \& Gies (2006a) have also observed that for the more massive stars of their sample (masses between 8.5 and $16 M_{\odot}$ ) there is an increase of the helium mass fraction at the surface of the star when the polar gravity decreases. The

\footnotetext{
${ }^{5}$ In that case, one passes from the $v \sin i$ values to the $v$ values by multiplying $v \sin i$ by $4 / \pi=1.27$. This is true only on average.
} 
increase of $Y$, the helium mass fraction, amounts to $23 \pm 13 \%$, between the zeroand terminal-age main sequences (i.e. if $Y=0.25$ on the zero-age main sequence, it reaches values as high as 0.31 on the terminal-age main sequence). Such helium enrichments at the surface have also been observed by Lyubimkov et al. (2004).

Does the velocity distribution vary as a function of metallicity? This question is still debated. For instance Keller (2004) measured the rotational velocities of 100 main sequence early B-type stars in clusters of the LMC, and compared them with those of early B-type stars in clusters of the solar neighbourhood. He found that the LMC stars are faster rotators than the Galactic ones: the mean value of $v \sin i$ is $116 \mathrm{~km} \mathrm{~s}^{-1}$ for the Galactic stars and $146 \mathrm{~km} \mathrm{~s}^{-1}$ for the LMC stars. On the other hand, Penny et al. (2004) found no difference between the velocities of O-type stars in the Magellanic Clouds and in the Galaxy. Numerous surveys are now being undertaken with the aim of providing further constraints on this topic, e.g. the VLT-Flames survey. The fact that the fraction of Be stars among B stars increases with decreasing metallicity (Be stars being stars near the break-up limit) may favour the situation where the distribution of initial rotations contains more fast rotators at low metallicity.

Rotation triggers many instabilities in stellar interiors. In turn these instabilities drive the transport of chemical species and of angular momentum in radiative zones. Typically on the main sequence, rotational diffusion will continually refuel the core in hydrogen, slowing down its mass decrease. At the end of the core $\mathrm{H}$ burning phase this will produce a more massive He core. The latter, in turn, will give birth to a more massive $\mathrm{CO}$ core. It may be easily deduced from the above line of reasoning that a rotating star will produce more ${ }^{16} \mathrm{O}$ than a non-rotating one.

In Table 3.13 we can see the effects of rotation on the yields of the three oxygen isotopes. For ${ }^{16} \mathrm{O}$, as emphasised above, the yields are increased by rotation (see also upper panel of Fig. 3.24). We note however that the increase is more important for the model at $Z=10^{-3}$ than for models at lower metallicities. For the other two isotopes, it is difficult to deduce any general behaviour from these data. We can just note that ${ }^{18} \mathrm{O}$ production is favoured in rotating models for metallicities below $10^{-5}$. This is related to the fact that in these models a strong peak of primary nitrogen is produced in the H-burning shell. Nitrogen from this peak diffuses into regions of lower nitrogen abundance, in particular in the He core where it is transformed into primary ${ }^{18} \mathrm{O}$ and ${ }^{22} \mathrm{Ne}$.

Interestingly, in plots of the yields versus the CO-core mass, models with and without rotation produce very similar curves (see Fig. 3.19). This illustrates well the fact that the size of the $\mathrm{CO}$ core is the determining factor. Of course rotation will change the correspondence between a given $\mathrm{CO}$ core mass and the initial mass.

Figure 3.22 shows the chemical yields from models with and without rotation (Hirschi et al. 2005) at $Z=0.020$. Figure 3.23 shows these yields multiplied by the stellar initial mass function (IMF). The main conclusion is that, below an initial mass of $30 M_{\odot}$, the cores are larger and thus the production of $\alpha$-elements is enhanced. Typically a rotating $20 M_{\odot}$ model will have more or less the same nucleosynthetic contribution as a non-rotating $30 M_{\odot}$ stellar model. This is true 

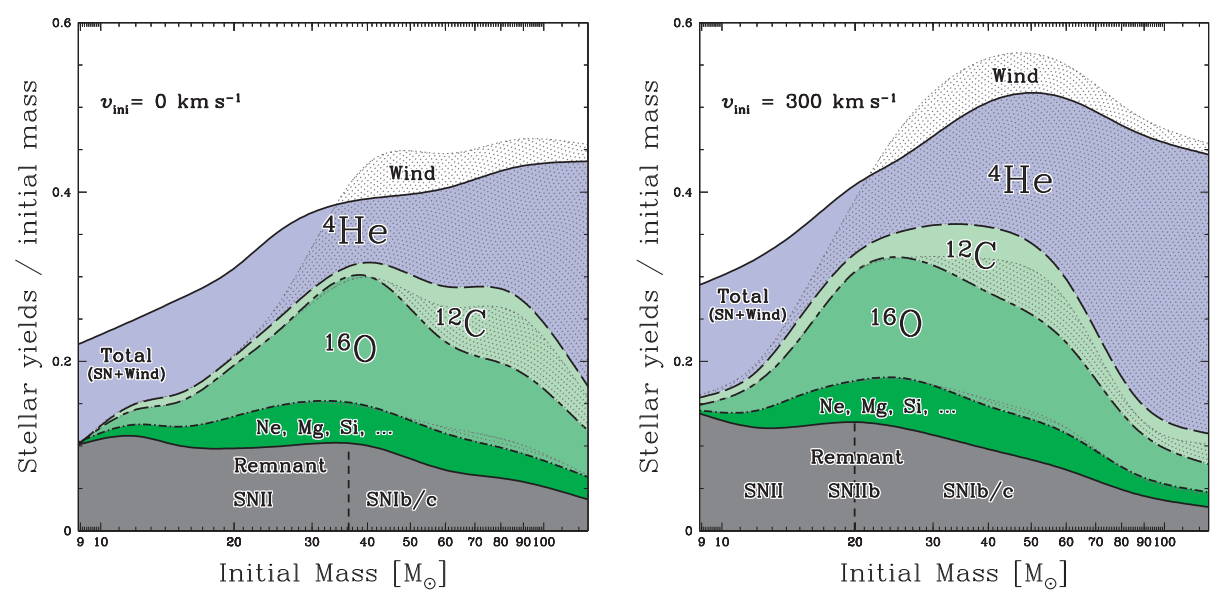

Fig. 3.22. Stellar yields. Top panel: models without rotation. Bottom panel: models with $v_{\text {ini }}=300 \mathrm{~km} \mathrm{~s}^{-1}$ (Hirschi et al. 2005). To illustrate how to read this figure, let us consider the case of the $60 M_{\odot}$ star without rotation. In that case, the mass in solar masses of new helium synthesised by the star is the length of the ${ }^{4} \mathrm{He}$ area at the $60 M_{\odot}$ abscissa $(0.4-0.28=0.12)$ multiplied by the initial mass of the star (here 60$)$. This gives about 7.2 $M_{\odot}$ of new helium produced by the star. The dotted areas correspond to the yields ejected by stellar winds only. Note that the total yield (wind and supernova) may be inferior to the yields ejected by winds in case the considered element is destroyed in the layers ejected at the time of the supernova explosion.
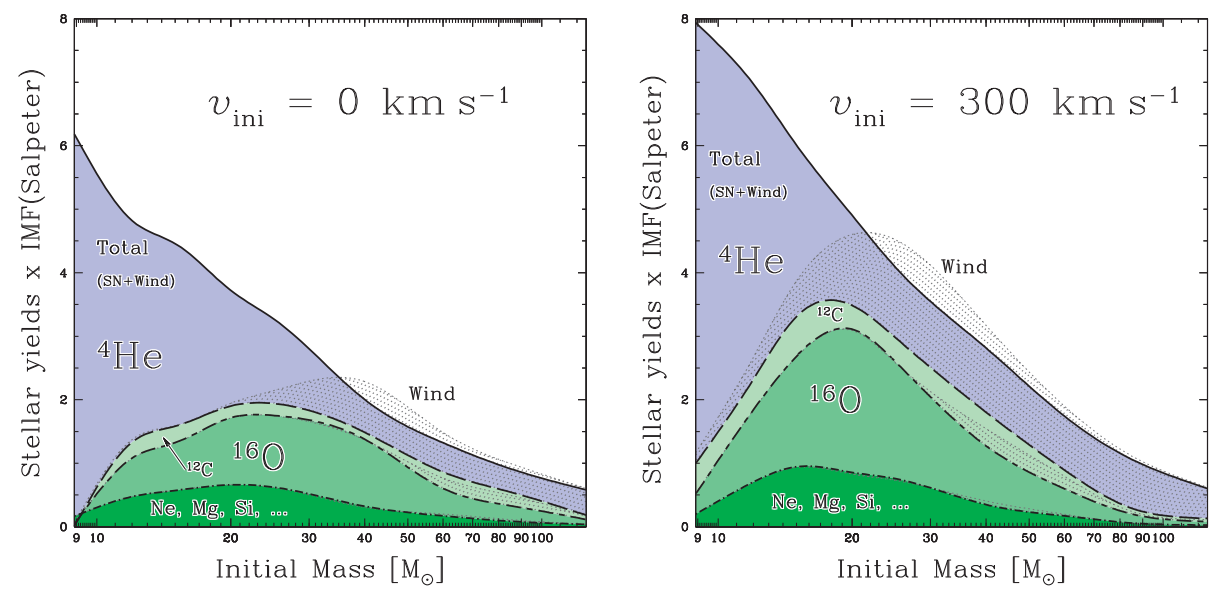

Fig. 3.23. IMF-weighted yields. Top panel: models without rotation. Bottom panel: models with $v_{\text {ini }}=300 \mathrm{~km} \mathrm{~s}^{-1}$ (Hirschi et al. 2005).

for velocities during the main sequence phase equal to the peak value of the velocity distribution. Above $30 M_{\odot}$, mass loss is the dominant effect and more He is ejected 
before being further processed, while the size of the core is only slightly reduced. When accounting for the weighting by the IMF, the production of oxygen and of $\alpha$-elements is globally enhanced as illustrated by Figure 3.23, while the effect on the He-production in massive stars remains limited.

\subsubsection{Yields}

In Figure 3.24, yields from different authors for the three stable isotopes of oxygen are shown as a function of the initial mass. All the models are computed for a metallicity $Z=0.02$. In the important mass range between 10 and $30 M_{\odot}$ the ${ }^{16} \mathrm{O}$ yields from the various authors agree well, while above this mass range great discrepancies are present. For the other two isotopes, large differences are found for the whole mass range considered in Figure 3.24.

\subsubsection{Observational probes}

Let us first begin by recalling a few observations supporting the now well-accepted fact that stars are indeed the sources of new synthesised elements. Historically one of the first observational hints that indeed stars were building new elements came from the detection of technetium at the surface of a red giant star (Merrill 1952). This radioactive element has indeed a disintegration lifetime $\left(2.13 \times 10^{5} \mathrm{y}\right)$ much shorter than the lifetime of the star in which it was observed. This clearly indicated that the origin of this element was related to processes occurring in the star itself. Today gamma-ray line observations allow the detection of gamma rays coming from the disintegration of radioactive elements. Up until now, five isotopes have been detected in this way: ${ }^{26} \mathrm{Al}$ (mean lifetime $1.04 \times 10^{6} \mathrm{y}$ ) and ${ }^{60} \mathrm{Fe}$ $\left(2.2 \times 10^{6} \mathrm{y}\right)$ have been detected as a diffuse emission in the Galactic disk, their abundances reflecting the global recent nucleosynthetic activity in the Galactic disk in the last million years; the emissions arising from the decay of ${ }^{44} \mathrm{Ti}$ ( $89 \mathrm{y}$ ) ${ }^{56} \mathrm{Co}(0.31 \mathrm{y})$ and ${ }^{57} \mathrm{Co}(1.1 \mathrm{y})$ have been observed from point sources linked to young supernova remnants ( $\mathrm{Ti}$ in Cas A, isotopes of Co in SN1987A) constraining the yield of individual events (see e.g. the review by Leising \& Diehl 2009).

The observation of surface abundances of non-radioactive elements is also an efficient probe for studying stellar evolution and checking the internal nuclear processes. Certainly one of the first findings supporting the view that massive stars are important oxygen producers is the observation that, in very metal-poor halo stars, the $[\mathrm{O} / \mathrm{Fe}]$ ratio is higher (by about a factor of 3 ) than that measured in stars with solar metallicity. This trend is attributed to the fact that in the very early time of the chemical enrichment of the Galaxy only the short-lived massive stars contribute. The fact that stars born in such an early time have an oxygen excess is an indication that the massive stars do produce great quantities of it (see Chapter 4).

Another argument is the fact that some stars, called Wolf-Rayet (WR) stars, offer the wonderful opportunity to look at what happens in their core. How is 


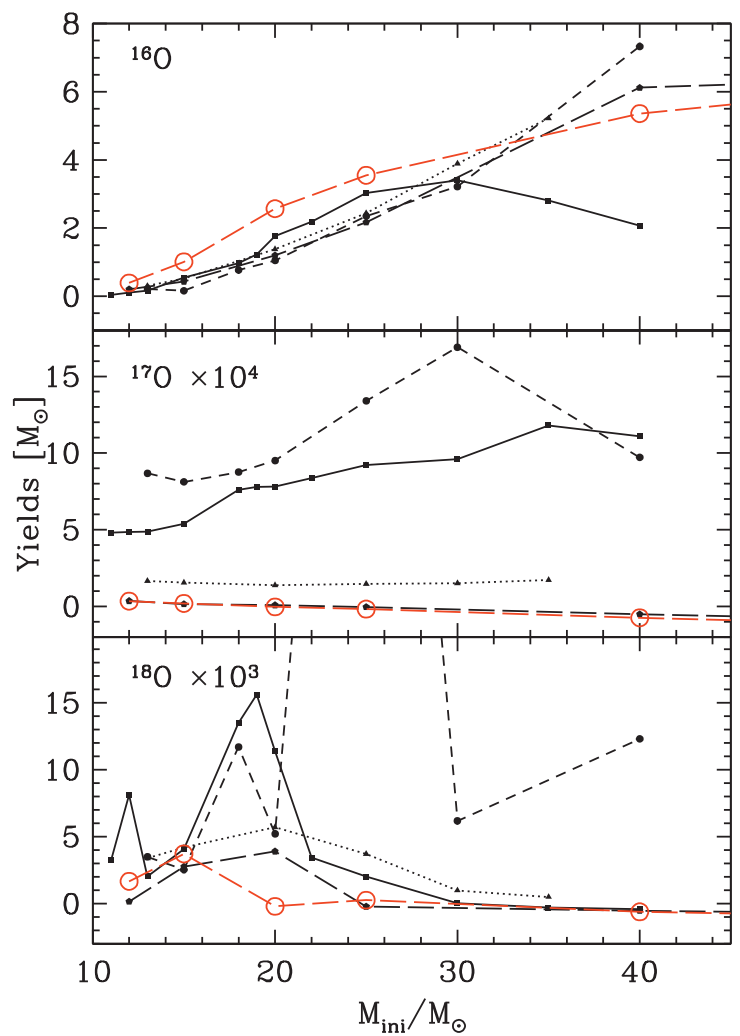

Fig. 3.24. Yields in ${ }^{16} \mathrm{O},{ }^{17} \mathrm{O}$ and ${ }^{18} \mathrm{O}$ for different initial models at a metallicity $Z=$ 0.02: the continuous curves with black squares corresponds to the yields from Woosley \& Weaver (1995); the dotted lines with black triangles present the results from Chieffi \& Limongi (2004); the dashed lines with black circles, those of Nomoto et al. (2006); the long dashed lines with black pentagons show the yields obtained by Hirschi et al. (2005) from non-rotating models; and the long dashed curve with large empty circles the yields from the same authors obtained from rotating models with $v_{\text {ini }}=300 \mathrm{~km} \mathrm{~s}^{-1}$. The yields are given in solar masses. The yields in ${ }^{17} \mathrm{O}$ and ${ }^{18} \mathrm{O}$ have been respectively multiplied by $10^{4}$ and $10^{3}$. Note that the results of Hirschi et al. (2005) correspond to yields obtained from pre-supernova models, i.e. the explosive nucleosynthesis is not accounted for. The other references account for the changes due to explosive nucleosynthesis. Note that for the isotopes considered here, the effect of the explosive nucleosynthesis is believed to be very modest.

this possible? Thanks to heavy mass loss and internal mixing processes, elements produced in the central regions appear at the surface thus offering an opportunity to check the nuclear processes occurring in stellar interiors (see the review on WR stars by Crowther 2007). 
Wolf-Rayet stars occupy the upper left-hand corner of the HR diagram and thus they are hot and luminous stars (see e.g. Hamann et al. 2006). At solar metallicity, they originate from stars more massive than about $25 M_{\odot}$ that have lost their initial $\mathrm{H}$-rich envelope by stellar winds or through a Roche lobe overflow in a close binary system. The stars enter the WR phase as WN stars, whose surface abundances are representative of equilibrium $\mathrm{CNO}$ processed material. If the peeling off continues, the star may enter the $\mathrm{WC} / \mathrm{WO}$ phase, during which the He-burning products appear at the surface. In our Galaxy, van der Hucht (2001, 2006) identifies $298 \mathrm{WR}$ stars (171 WN, $10 \mathrm{WN} / \mathrm{WC}, 113 \mathrm{WC}$ and $4 \mathrm{WO})$, of which $24(8 \%)$ are in the open cluster Westerlund 1 and $60(20 \%)$ are in open clusters near the Galactic Centre. One estimates that their total number in our Galaxy is as high as a few thousands.

The observed surface abundances during the WN phase correspond to CNO equilibrium values, while those observed during the $\mathrm{WC} / \mathrm{WO}$ phase well correspond to the apparition at the surface of He-burning products (Crowther 2007). Oxygen diagnostics in WC stars lie in the near UV and are thus difficult to observe from the ground. Thanks to space-based spectroscopy, Crowther et al. (2002) derived an oxygen mass fraction of $5-10 \%$ in WC stars. Such a high oxygen value (let us recall that at solar metallicity oxygen has a mass fraction of at most 1\%) beautifully supports the view that indeed oxygen is a product of helium burning.

Although a little aside from the subject of oxygen nucleosynthesis, it is interesting to mention the following: WC stars offer a neat and original way to measure the initial CNO abundance. At first sight, this assertion looks quite strange. How can material heavily processed by at least two nuclear phases ( $\mathrm{H}$ - and He-burning reactions) still provide information on initial conditions? The reason is that during the core $\mathrm{H}$-burning phase $\mathrm{CNO}$ are transformed into $\mathrm{N}$; therefore, $\mathrm{N}$ at this stage is a measure of the initial CNO content. During the core He-burning phase, this $\mathrm{N}$ is converted into ${ }^{22} \mathrm{Ne}$, and when the He core is naked, the measure of the neon abundance then gives access to the initial CNO content of the star.

The high overabundance of ${ }^{22} \mathrm{Ne}$ at the surface of the WC star predicted by Heburning reactions is well confirmed by observations (see e.g. Ignace et al. 2007). But comparisons between the observed $\mathrm{Ne} / \mathrm{He}$ ratio at the surface of WC stars with models computed with $Z=0.02$ show that the latter overpredict the $\mathrm{Ne}$ abundance, while models starting with $Z=0.014$ give a much better fit as can be seen in Figure 3.25. This is an indirect argument that the solar abundances of Asplund et al. (2004) represent much better the metallicity of the solar vicinity than previous estimates (see Chapter 2).

Let us note that this overabundance of ${ }^{22} \mathrm{Ne}$ at the surface of WC stars is not only an important confirmation of the nuclear reaction chains occurring during He-burning, but it is also related to the question of the origin of the material accelerated into Galactic cosmic rays (see recent measurements of the ${ }^{22} \mathrm{Ne} /{ }^{20} \mathrm{Ne}$ ratio in cosmic rays in Binns et al. 2005) and to the weak s-process in massive stars since ${ }^{22} \mathrm{Ne}$ is the source of neutrons in these stars.

Let us end this section by mentioning an interesting approach to constrain the chemical composition of core-collapse supernova ejecta (very rich in oxygen). 


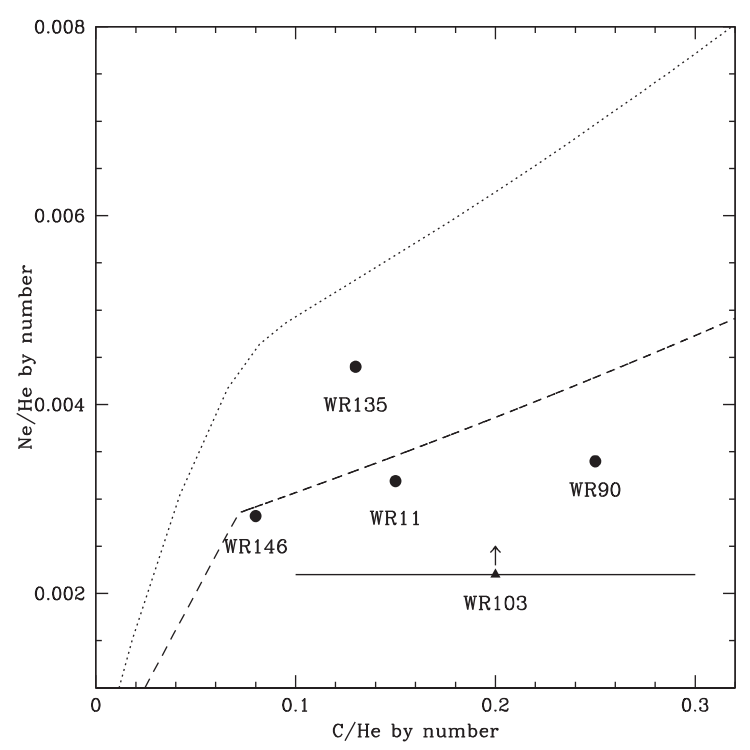

Fig. 3.25. The black points show the Ne and C abundances observed at the surface of WC stars by Dessart et al. (2000; filled circles) and by Crowther et al. (2006; filled triangles). The dotted line shows the prediction for a $60 M_{\odot}$ stellar model with $Z=0.020$ of Meynet \& Maeder (2003) and the dashed line for a $60 M_{\odot}$ stellar model with $Z=0.014$.

When a core collapse occurs in a binary system, there is a chance that part of the ejecta will be intercepted by the companion. In that case the companion may present surface abundances different from those of nearby stars. At the present time three such cases have been observed (see e.g. González Hernández et al. 2009). For instance large overabundances (factors between 6 to 10 with respect to solar values) of oxygen, magnesium, silicon and sulphur have been observed in the atmosphere of the star orbiting a probable black hole, Nova Scorpii 1994. This is the first observational evidence of a supernova event being associated to the birth of a black hole. This also indicates that not all the mass is swallowed by the black hole, but a fraction of it participates in the chemical enrichment of the surroundings. Finally this observation also supports the view that at the time of the supernova event large quantities of oxygen are ejected into the interstellar medium.

\subsubsection{Core-collapse supernovae}

What are the effects of explosive nucleosynthesis on the yields of the oxygen isotopes? To answer this question we may compare the yields obtained at the presupernova stage with those obtained after the passage of the shock wave in the outer layer when the star explodes as a supernova. Woosley \& Weaver (1995) provide such numbers for a $25 M_{\odot}$ star at solar metallicity. The ejected masses before the explosive nucleosynthesis are $3.45 M_{\odot}$ for ${ }^{16} \mathrm{O}, 1.01 \times 10^{-3} M_{\odot}$ for ${ }^{17} \mathrm{O}$ 
and $2.72 \times 10^{-3} M_{\odot}$ for ${ }^{18} \mathrm{O}$, and $3.25 M_{\odot}$ for ${ }^{16} \mathrm{O}, 1.01 \times{ }^{-3} M_{\odot}$ for ${ }^{17} \mathrm{O}$ and $2.52 \times 10^{-3} M_{\odot}$ for ${ }^{18} \mathrm{O}$ after the explosion. Thus the effects of the explosive nucleosynthesis are very modest (at least for this stellar mass). They are larger for elements produced in the regions lying closer to the centre, as expected, since it is in these regions that the higher values of temperature and density are reached.

The most important effect of supernova explosion on the yields is actually through the mass cut, i.e. the dividing line between the mass which will remain locked in the compact remnant and that ejected at the time of the supernova event. As already outlined above, in the case of the $25 M_{\odot}$ star discussed in Section 3.2.2.1, if the mass cut is below $2.5 M_{\odot}$, the yields of ${ }^{16} \mathrm{O}$ are little affected (see Fig. 3.18). If the mass cut is above $2.5 M_{\odot}$, then its exact value has important consequences: the mass of ${ }^{16} \mathrm{O}$ ejected decreases with increasing mass cut by about $0.07 M_{\odot}$ per tenth of solar mass.

\subsubsection{Pair-instability supernovae}

Very massive stars which form a helium core of about $40 M_{\odot}$ or larger experience the electron-positron pair instability (Barkat et al. 1967; Bond et al. 1984). Physically this instability comes from the fact that, in the advanced phases of the evolution, photons have sufficient energy to produce electron-positron pairs. In these very massive stars, the hydrostatic equilibrium is mostly sustained by the radiative pressure gradient. Since the process of pair formation removes important agents sustaining the star (i.e. photons), it contributes to make the star unstable with respect to contraction: the star rapidly collapses. The acceleration of the nuclear energy reaction rates due to neon, oxygen and silicon burning may then turn the collapse into an explosion.

For helium core masses between 40 and $63 M_{\odot}$, the energy released is not sufficient to disrupt the star and the star becomes pulsationally unstable (Heger \& Woosley 2005). The envelope and part of the He core are ejected, and a black hole is formed. For helium core masses between 63 and $130 M_{\odot}$, the released energy is enough to completely disrupt the stars. Above $130 M_{\odot}$, photodisintegration in the cores lead to black-hole formation.

Pair-instability supernovae only occur if the progenitor star can retain sufficient mass at the end of its evolution to encounter the instability described above. This is the reason why these events are believed to take place only in very metal-poor stars where line driven winds are very weak. For instance it was expected to see the signature of pair-instability supernovae in the very iron poor stars observed in the halo; however no such signature has yet been found (Cayrel et al. 2004).

Recently the observation of very luminous supernovae presenting many observed characteristics compatible with pair-instability supernovae suggested that in the present-day Universe such events may occur. However recent near-infrared observations of the SN 2006gy, for instance, show that the luminosity curve presents a late-time decline which is not in agreement with the various pair-instability supernova models (Miller et al. 2010). 
Thus at the moment, although the physics of such explosions is well understood and their nucleosynthetic outputs well described, any direct link with observed features remains elusive. It is thus difficult to quantitatively estimate the importance of such events in the synthesis of oxygen. At the moment it seems that their main contribution was in the very early Universe, although even at that time stars may have lost high amounts of mass due to fast rotation (Ekstroem et al. 2008) and thus avoided the pair instability. Yields from pair-instability supernova models can be found in Heger \& Woosley (2005).

\subsubsection{Low- and intermediate-mass stars}

Low- and intermediate-mass stars finish their lives as carbon-oxygen white dwarfs. Their initial masses are typically between $0.5 M_{\odot}$ (less massive stars burn only hydrogen and end up as helium white dwarfs) and 6-8 $M_{\odot}$ (more massive stars go through all the nuclear phases beyond helium burning as discussed in Sect. 3.2.2). After having gone through the central hydrogen-burning phase that is referred to as the main sequence, these objects undergo a short period of shell hydrogen burning on the red giant branch followed by core helium burning on the so-called clump ${ }^{6}$. When the helium fuel is consumed in the central region, low- and intermediatemass stars evolve to the asymptotic giant branch (hereafter AGB). The electrondegenerate core of carbon and oxygen, which is the product of helium burning, is then surrounded by a helium-burning shell and a more external hydrogen-burning shell. From this time on, recurrent thermal pulses take place in the helium-burning shell. This is the so-called the TP-AGB phase, which provides a rich and unique nucleosynthesis. Moreover, recurrent dredge-up events (third dredge-up) enrich the stellar surface with the freshly synthesised nuclides that are then ejected into the interstellar medium through the strong winds (prior to the TP-AGB, the surface composition of the stars has also been altered by the first and possibly second dredge-up events). Through these mechanisms, low- and intermediatemass stars thus play a crucial role in the chemical evolution of galaxies. Let us now have a closer look at their contribution to the oxygen isotopes.

\subsubsection{A reference case: a $5 M_{\odot}$ star}

We focus first on a reference object, a $5 M_{\odot}$ star with metallicity $[\mathrm{Fe} / \mathrm{H}]=-1.56$ (from Decressin et al. 2009). In this model the effects of rotation are not included.

\section{Abundance profiles at the end of the main sequence}

Figure 3.26 (top) shows the abundance profiles of different chemical species inside the star at the end of the main sequence. In regions deeper than $\mathrm{M}_{r} / M_{\odot} \sim 3$,

\footnotetext{
${ }^{6}$ Low- and intermediate-mass stars respectively ignite central helium burning in electrondegenerate and non-degenerate cores. The mass limit is approximately $2-2.2 M_{\odot}$ and depends on the initial stellar metallicity.
} 

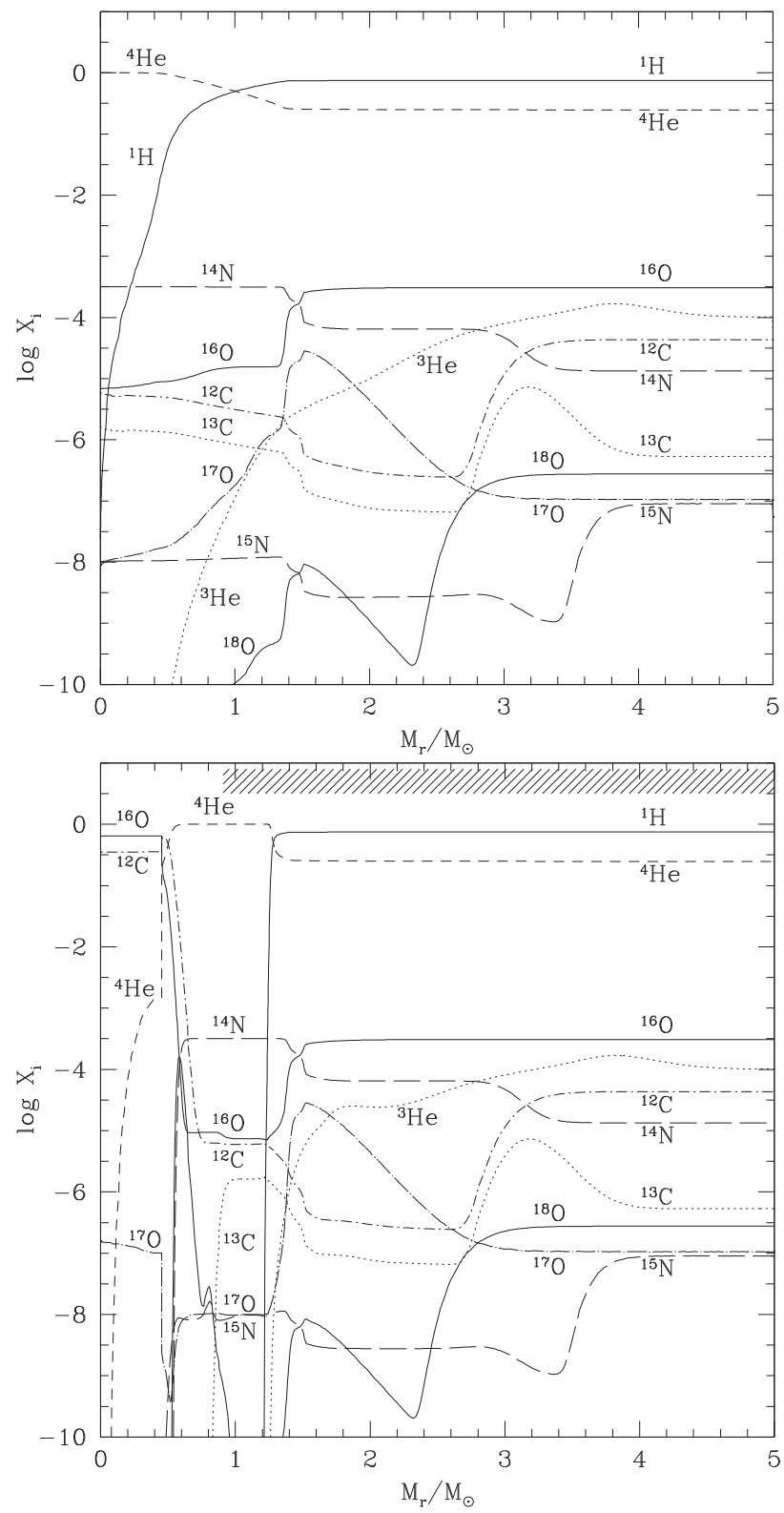

Fig. 3.26. Abundance profiles (in mass fraction) of chemical elements as a function of depth (reduced mass in solar units) inside the reference $5 M_{\odot},[\mathrm{Fe} / \mathrm{H}]=-1.56$ model. Top: at the end of the main sequence. Bottom: at the end of the core He-burning phase. The hatched area indicates the maximum extent of the convective envelope during the dredge-up episode that occurs early on the AGB. 
the oxygen isotopes are affected by the $\mathrm{ON}$ cycle, ${ }^{16} \mathrm{O}$ and ${ }^{18} \mathrm{O}$ being destroyed by p-captures and $\alpha$-decay, while ${ }^{17} \mathrm{O}$ is produced at a high rate. In the most central regions where the $\mathrm{CNO}$ cycle has reached equilibrium the three oxygen isotopes are depleted.

\section{Abundance profiles at the end of core He burning and dredge-up}

Figure 3.26 (bottom) shows the chemical structure of the star at the end of central helium burning. In the core $\left(\mathrm{M}_{r} / M_{\odot}<0.45\right)$, helium has been transformed into ${ }^{12} \mathrm{C}$ and ${ }^{16} \mathrm{O}$ through $3 \alpha$ and ${ }^{12} \mathrm{C}(\alpha, \gamma){ }^{16} \mathrm{O}$ reactions. At the edge of the core $\left(0.45<\mathrm{M}_{r} / M_{\odot}<1.5\right)$ the three oxygen isotopes are depleted due to hydrogen burning through the $\mathrm{CNO}$ cycle in a shell. In the most external regions $\left(\mathrm{M}_{r} / M_{\odot}>\right.$ 1.5) the abundance profiles have not been modified since the end of the main sequence.

Following core He exhaustion, the star starts ascending the AGB, and its convective envelope deepens down to the nuclearly processed regions as indicated by the hatched area in Figure 3.26 (bottom). This is the so-called second dredge-up ${ }^{7}$ that leads to changes in the stellar surface abundances. In the model presented here, ${ }^{16} \mathrm{O} /{ }^{17} \mathrm{O}$ then decreases by a factor of $\sim 26$ (the convective envelope totally engulfs the ${ }^{17} \mathrm{O}$ peak) while ${ }^{16} \mathrm{O} /{ }^{18} \mathrm{O}$ increases by a factor of $\sim 1.6$. The fresh ${ }^{16} \mathrm{O}$ produced in the core is not affected by the dredge-up and remains trapped into the degenerate core of the future white dwarf. Finally, let us note that during the dredge-up episode on the early-AGB the sum $\mathrm{C}+\mathrm{N}+\mathrm{O}$ at the surface of this standard (non-rotating) model remains constant, since only H-burning products are conveyed to the surface.

\section{TP-AGB phase}

Later on helium and hydrogen burnings ignite inside two thin layers surrounding the contracting degenerate carbon-oxygen core. These burning shells become thinner, hotter and closer to each other with time, and their ashes increase the core mass. Soon a first thermal instability (or thermal pulse) occurs in the heliumburning shell, which marks the beginning of the TP-AGB. This evolution phase is characterised by long periods of quiescent H-shell burning (or inter-pulses), interrupted by recurrent thermal pulses of the helium-burning shell. The enormous quantity of energy produced by each pulse powers a convective pocket that engulfs the ashes of the hydrogen-burning shell causing its momentary extinction. When the thermal pulse dies down, the stellar convective envelope moves inward in mass and eventually reaches the region previously mixed by the convective pocket. This is known as the third dredge-up, which conveys the products of partial helium burning to the surface. Following each thermal pulse the hydrogen-burning shell

\footnotetext{
${ }^{7}$ For stars less massive than $2-2.5 M_{\odot}$, a first dredge-up episode already occurs at the tip of the red giant branch, see Section 3.2.3.2.
} 
reignites and the star enters a new inter-pulse period. The cycle inter-pulse - thermal pulse - third dredge-up occurs several times on the TP-AGB, depending on the initial mass and composition of the star. It ends up when the stellar envelope has been entirely ejected by the important mass loss that the star undergoes at this stage of its evolution. In the most massive AGB stars such as the reference model discussed here, the convective envelope dips into the regions of $\mathrm{H}$ burning, leading to nuclear processing at the base of the envelope. This is the so-called hot bottom burning, which leads to modification of the surface abundance when the temperature at the base of the convective envelope exceeds $\sim 50$ million $\mathrm{K}$. As first investigated by Boothroyd et al. (1993), hot bottom burning can efficiently reduce the ${ }^{12} \mathrm{C} /{ }^{16} \mathrm{O}$ ratio at the stellar surface and, if it is strong enough, it possibly prevents the formation of $\mathrm{C}$ stars. During the TP-AGB, three different nucleosynthesis sites thus have to be distinguished: the burning shells, the convective region where the thermal pulse develops and the base of the convective envelope.

Figure 3.27 shows the abundance profiles of key elements in the tiny region of nuclear activity (i.e., helium- and hydrogen-burning shells) in our reference model (initial mass of $5 M_{\odot}$ and $[\mathrm{Fe} / \mathrm{H}]=-1.56$ ) at the end of the inter-pulse phase after the 14th thermal pulse. The nuclear energy supplied by the helium-burning shell comes from the $3 \alpha(\sim 70 \%)$ and ${ }^{12} \mathrm{C}(\alpha, \gamma)(\sim 29 \%)$ reactions. The helium-burning shell produces ${ }^{12} \mathrm{C}$ and, to a lower extent, ${ }^{16} \mathrm{O}$, while during the previous central He-burning stage the ${ }^{16} \mathrm{O}$ production was preponderant. In addition ${ }^{16} \mathrm{O},{ }^{17} \mathrm{O}$ and ${ }^{18} \mathrm{O}$ are partially destroyed in the hydrogen-burning shell.

During the next thermal pulse part of the inter-shell region is engulfed by the convective tongue. ${ }^{17} \mathrm{O}$, which is not produced inside thermal pulses, is then destroyed mainly through ${ }^{17} \mathrm{O}(\alpha, \mathrm{n})$ and ${ }^{17} \mathrm{O}(\alpha, \gamma)$, the first reaction being at least ten times faster than the second and weakly contributing to neutron production inside the thermal pulses. ${ }^{17} \mathrm{O}$ destruction already operates from the first thermal pulses on, whatever the initial stellar mass and metallicity. In subsequent thermal pulses, the ${ }^{17} \mathrm{O}$ mass fraction stabilises around 4 to $8 \times 10^{-10}$, which represents a balance between the amount of ${ }^{17} \mathrm{O}$ removed from the inter-shell region and that destroyed by the thermal pulse.

On the other hand ${ }^{18} \mathrm{O}$ is produced inside the thermal pulse via the ${ }^{14} \mathrm{~N}(\alpha, \gamma){ }^{18} \mathrm{~F}$ reaction and the rapid ${ }^{18} \mathrm{~F} \beta$-decay to ${ }^{18} \mathrm{O}$ which significantly contributes to the thermal pulse energetics. This isotope is destroyed by three reactions: ${ }^{18} \mathrm{O}(\alpha, \gamma)$, ${ }^{18} \mathrm{O}(\alpha, \mathrm{n})$ and ${ }^{18} \mathrm{O}(\mathrm{p}, \alpha){ }^{15} \mathrm{~N}$ in order of decreasing nuclear timescales (although the third reaction can become faster than the first one when significant amounts of protons are produced). Since the convective tongues heat up during the TP-AGB, ${ }^{18} \mathrm{O}$ destruction becomes more and more efficient from pulse to pulse.

We can now identify which specific nuclear region contributes to change the abundance of each oxygen isotope inside a TP-AGB star. ${ }^{16} \mathrm{O}$ is depleted in the inter-shell region, but is produced inside the thermal pulses. During third dredgeup events, its surface abundance increases very slightly. ${ }^{17} \mathrm{O}$ is always considerably depleted in both the inter-shell and thermal pulse regions, so that the third dredge-up decreases its surface abundance. Finally, at the end of full amplitude thermal pulses, ${ }^{18} \mathrm{O}$ has a lower abundance than within the convective envelope. 


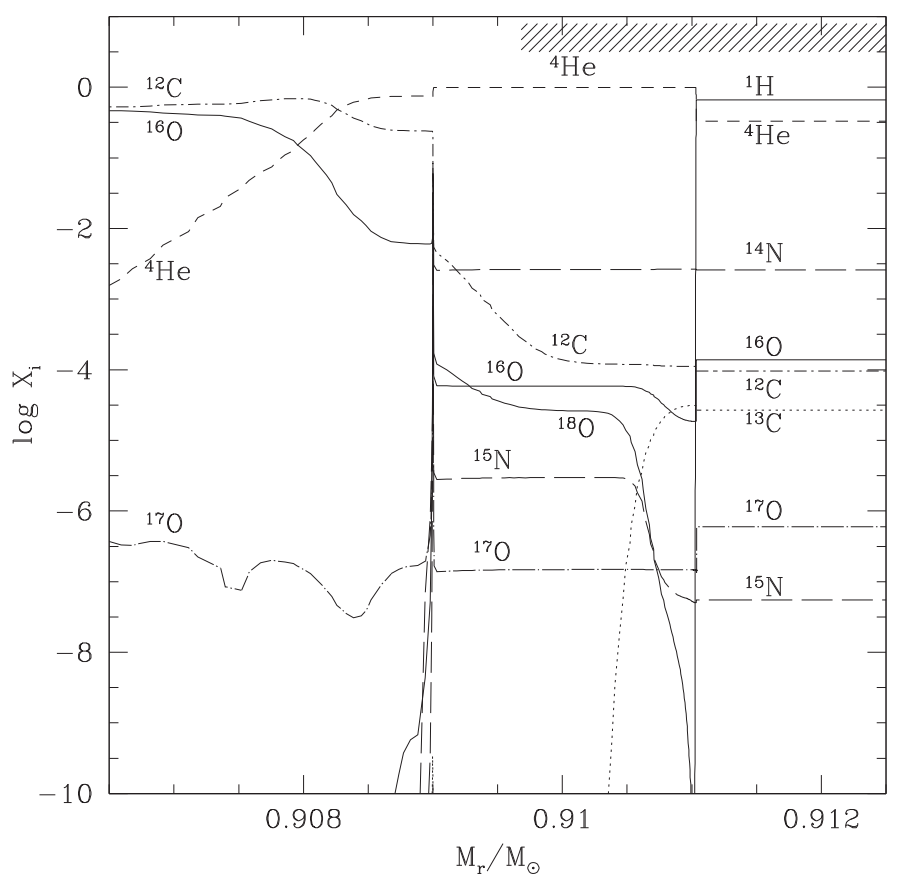

Fig. 3.27. Abundance profiles in the internal zone of the reference AGB star (initial mass of $\left.5 M_{\odot},[\mathrm{Fe} / \mathrm{H}]=-1.56\right)$ at the end of the inter-pulse phase just before the occurrence of the 15 th thermal pulse. At that stage the total mass of the star is $3.53 M_{\odot}$. The helium-burning region extends from $\mathrm{M}_{r} / M_{\odot} \sim 0.904$ up to $\sim 0.909$, and is surrounded by the inter-shell region that contains the ashes of shell hydrogen burning. The temperature at the base of the convective envelope (located at $\mathrm{M}_{r} / M_{\odot} \sim 0.911$ ) is $\sim 89 \times 10^{6} \mathrm{~K}$, so that hot bottom burning is efficient at that stage. The hatched area on top indicates the maximum extent of the convective envelope during the next dredge-up episode. During the forthcoming 15 th pulse, the convective tongue will extent up to $\mathrm{M}_{r} / M_{\odot} \sim 0.911$.

Its abundance thus drastically decreases from third dredge-up to third dredge-up. Consequently, both ${ }^{16} \mathrm{O} /{ }^{17} \mathrm{O}$ and ${ }^{16} \mathrm{O} /{ }^{18} \mathrm{O}$ surface isotopic ratios increase during each third dredge-up episode. On the other hand if hot bottom burning occurs, the operation of the $\mathrm{ON}$ cycle in the convective envelope increases the amount of ${ }^{17} \mathrm{O}$ at the expense of ${ }^{16} \mathrm{O}$, and substantially destroys ${ }^{18} \mathrm{O}$ at the stellar surface. So in massive AGB stars, such as the reference case presented here, ${ }^{16} \mathrm{O} /{ }^{17} \mathrm{O}$ can decrease after each thermal pulse while ${ }^{16} \mathrm{O} /{ }^{18} \mathrm{O}$ increases much more than in lower-mass stars that do not experience hot bottom burning.

Figure 3.28 presents the evolution of the surface abundances and isotopic ratios until the end of the TP-AGB phase in the reference model. The abscissa is the total stellar mass, which decreases due to strong mass loss on the TP-AGB and 

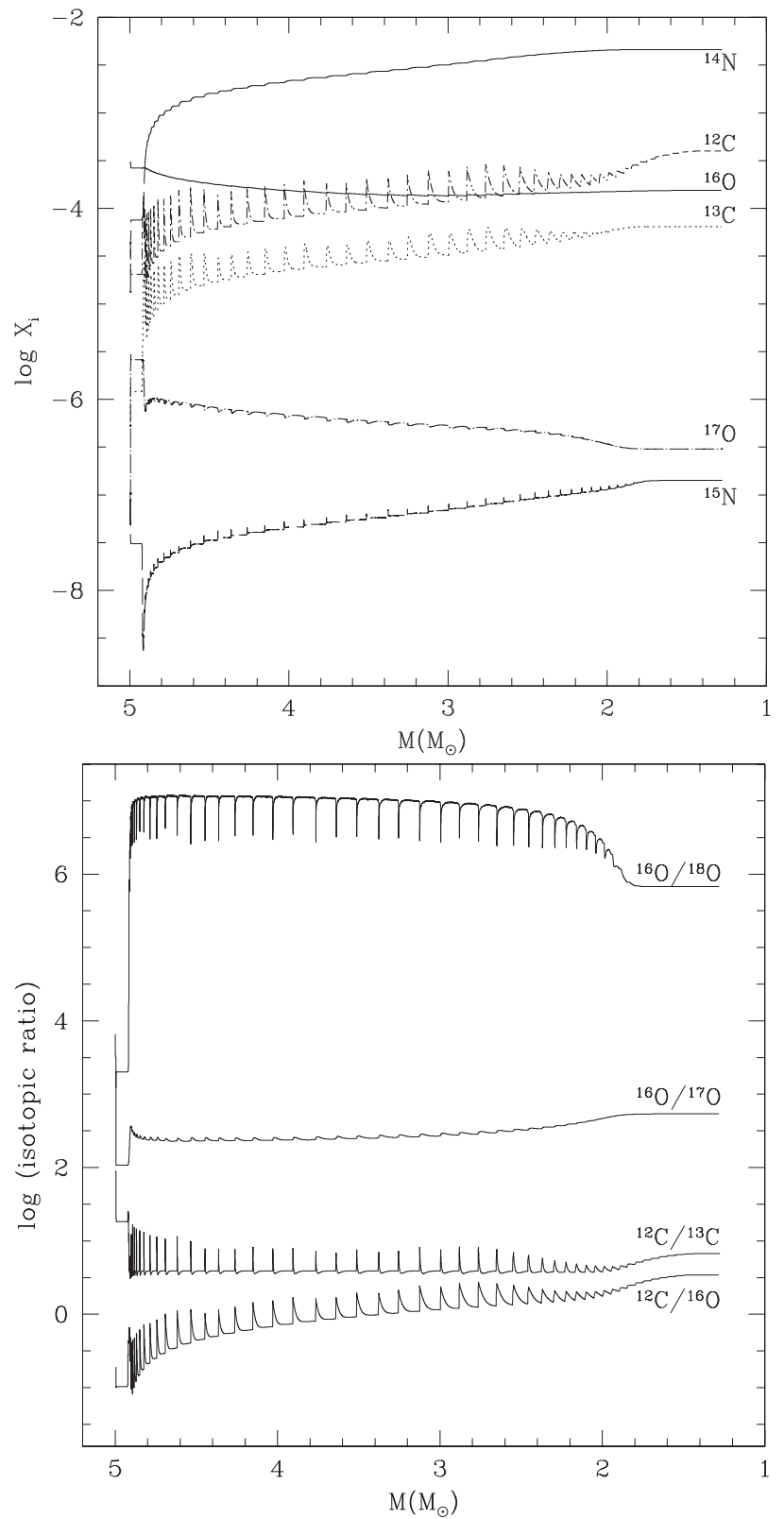

Fig. 3.28. Bottom: evolution of the surface abundances (logarithm of the mass fraction) of some elements as a function of remaining total stellar mass (in solar units) for our reference model (initial mass of $5 M_{\odot}$ and $[\mathrm{Fe} / \mathrm{H}]=-1.56$ ). Top: same for isotopic ratios. 
depicts time. Peaks on the tracks indicate the occurrence of successive thermal pulses and of associated third dredge-up and hot bottom burning episodes.

\subsubsection{Effects of mass, metallicity, rotation, etc.}

Mass and metallicity. The chemical profiles inside a star at a given evolutionary phase depend both on stellar mass and metallicity, as shown at the end of central hydrogen and helium burning in Figure $3.29\left(2.5 M_{\odot},[\mathrm{Fe} / \mathrm{H}]=-1.56\right)$ and Figure 3.30 ( $5 M_{\odot}$, solar metallicity), which can be compared to Figure 3.26 (5 $M_{\odot},[\mathrm{Fe} / \mathrm{H}]=-1.56$, reference model). The more massive a star at a given metallicity, or the less metallic a star at a given initial mass, the larger the temperature, pressure and density at a given depth, which induces a shift of the chemical profiles towards a more external region in terms of $\mathrm{M}_{r} / \mathrm{M}_{*}$.

In the case of low-mass stars $\left(\mathrm{M} \leq 2-2.2 M_{\odot}\right)$ that ignite central He burning via a flash in the degenerate core at the tip of the red giant branch, a first dredge-up already modifies the surface abundances at the base of the red giant branch. The convective envelope expands inwards, and reaches the regions that have been previously nuclearly processed (i.e. both during central and thick shell $\mathrm{H}$ burnings), its maximum expansion decreasing with increasing initial stellar mass (Boothroyd \& Sackmann 1999; Charbonnel 1994; El Eid 1994). The first dredge-up leads to an increase of the surface abundance of ${ }^{17} \mathrm{O}$ and a decrease of ${ }^{16} \mathrm{O}$ and ${ }^{18} \mathrm{O}$, the amplitudes of which depend on the initial stellar mass as shown in Figure 3.31. For initial stellar masses lower than $2.5 M_{\odot}$, the predicted post dredge-up ${ }^{16} \mathrm{O} /{ }^{18} \mathrm{O}$ ratio slightly increases with respect to its initial value (by up to $\sim 30-40 \%$ ), while the post dredge-up ${ }^{16} \mathrm{O} /{ }^{17} \mathrm{O}$ ratio is substantially diminished from its initial value. This depends crucially on the maximum depth reached by the convective envelope, i.e. on how far the ${ }^{17} \mathrm{O}$ peak is engulfed by the convective envelope.

Thermohaline mixing. Numerous observations provide compelling evidence of a non-canonical mixing process that occurs when low-mass stars reach the so-called bump in luminosity on the red giant branch. At that phase, indeed, the surface carbon isotopic ratio drops, together with the abundances of lithium and carbon, while that of nitrogen increases slightly (see references in Charbonnel \& Lagarde 2010). Thermohaline mixing has recently been identified as the mechanism that governs the photospheric composition of low-mass bright giants (Charbonnel \& Zahn 2007). In these stars, this double-diffusive instability is induced by the molecular weight inversion created by the ${ }^{3} \mathrm{He}\left({ }^{3} \mathrm{He}, 2 \mathrm{p}\right){ }^{4} \mathrm{He}$ reaction in the external wing of the hydrogen-burning shell. Among the oxygen isotopes, only ${ }^{18} \mathrm{O}$ is affected by this mechanism, which leads to a slight increase of the ${ }^{16} \mathrm{O} /{ }^{18} \mathrm{O}$ surface ratio compared to the post dredge-up value, while the ${ }^{16} \mathrm{O} /{ }^{17} \mathrm{O}$ ratio does not change (Charbonnel \& Lagarde 2010).

Rotation. We show in Figure 3.32 the abundance profiles at the end of central hydrogen and helium burning for a $5 M_{\odot},[\mathrm{Fe} / \mathrm{H}]=-1.56$ model computed taking into account the effects of rotation as described within the formalism developed by 

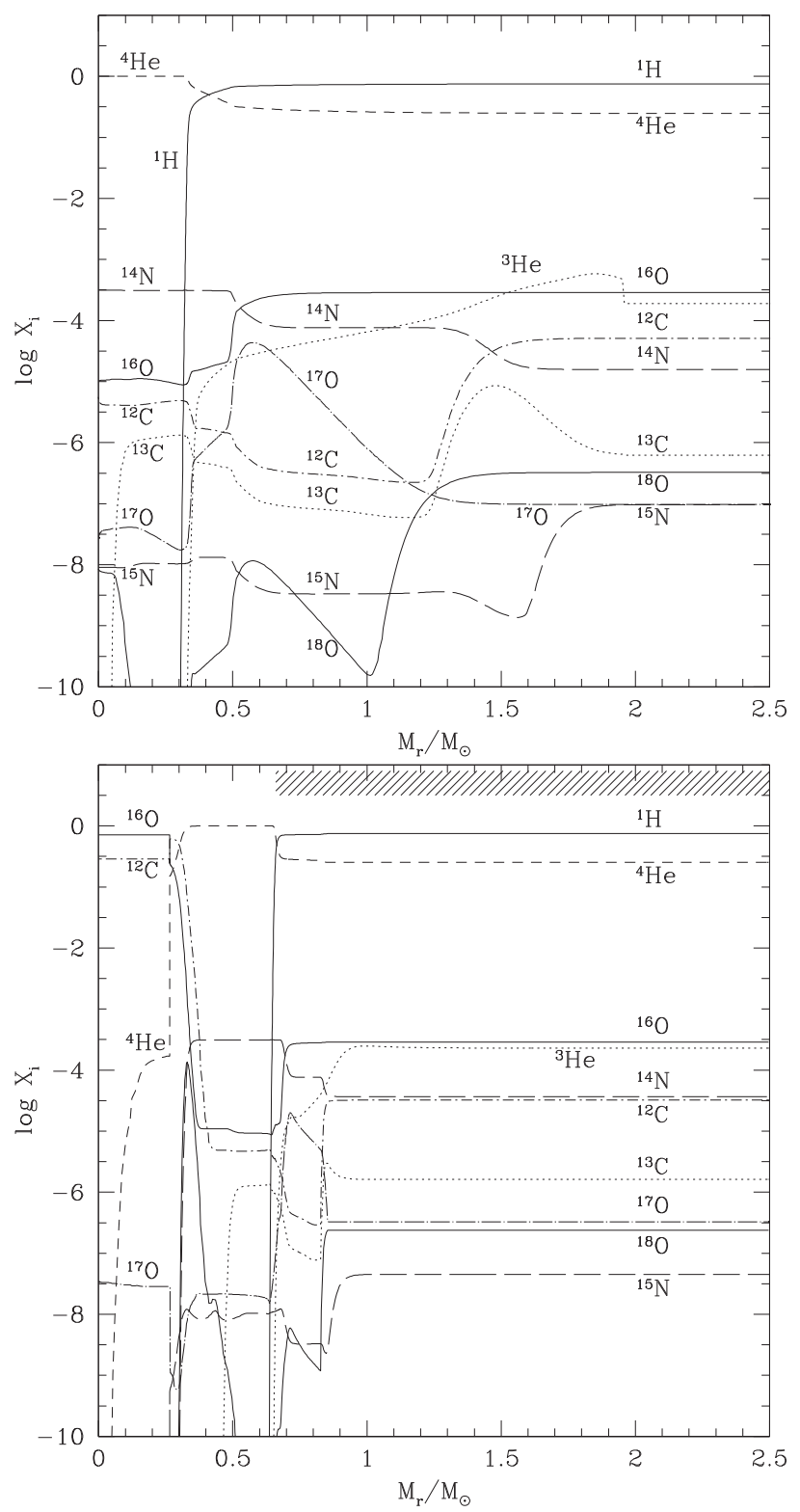

Fig. 3.29. Same as Figure 3.26 for a star with an initial mass of $2.5 M_{\odot}$ and $[\mathrm{Fe} / \mathrm{H}]=-1.56$.

Zahn (1992) and Maeder \& Zahn (1998) (see Sect. 3.2.2 and Decressin et al. 2009, for more details). The initial rotation velocity on the zero-age main sequence for 

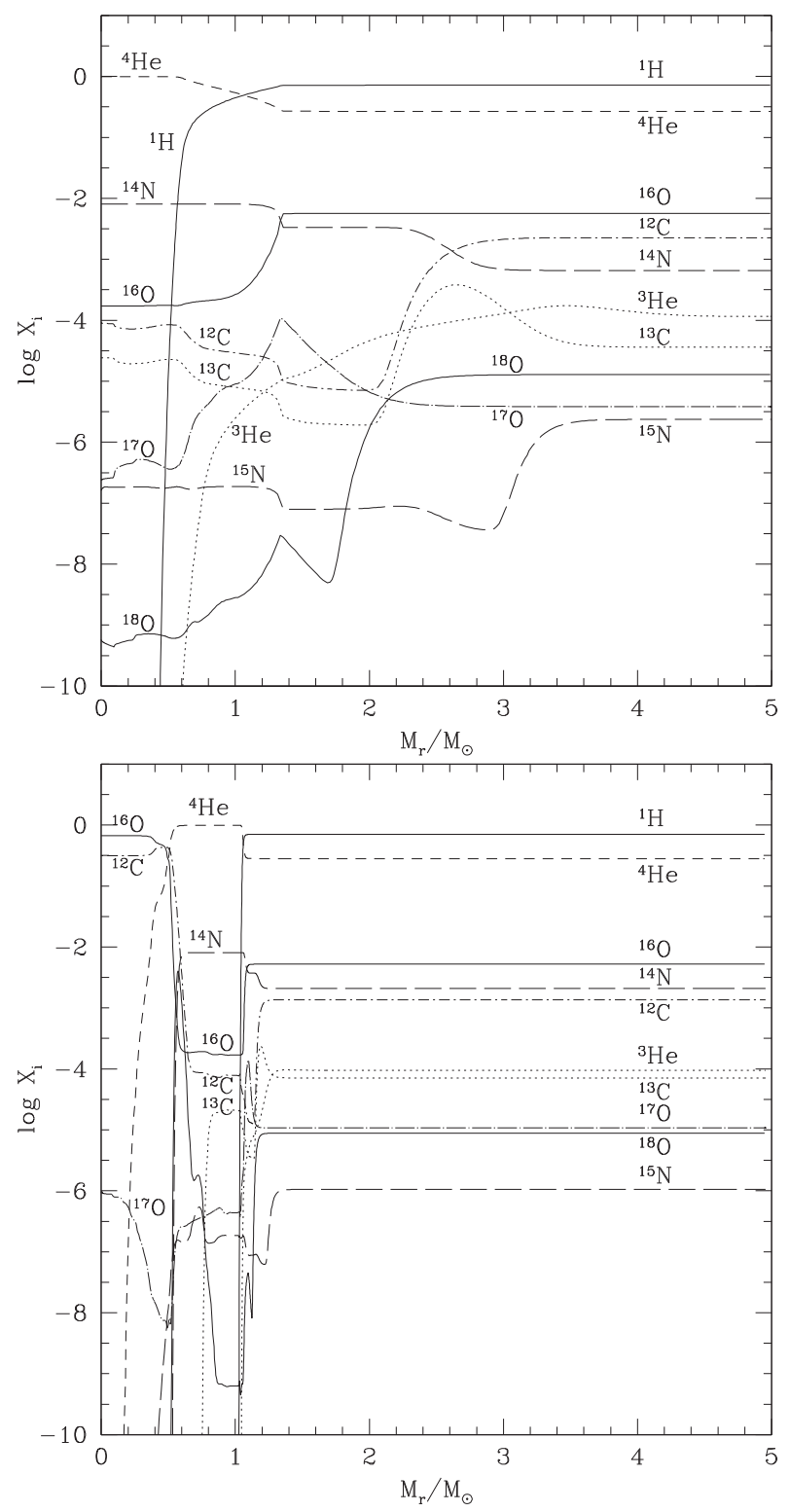

Fig. 3.30. Same as Figure 3.26 for a star with an initial mass of $5 M_{\odot}$ at solar metallicity. At the end of central He burning (bottom) the convective envelope has already started deepening in mass and homogenising the external layers down to $\mathrm{M}_{r} / M_{\odot} \sim 1.2$.

this model is $300 \mathrm{~km} \mathrm{~s}^{-1}$. Rotation-induced mixing strongly modifies the internal chemical structure compared to the standard case (compare Figs. 3.26 and 3.32). 


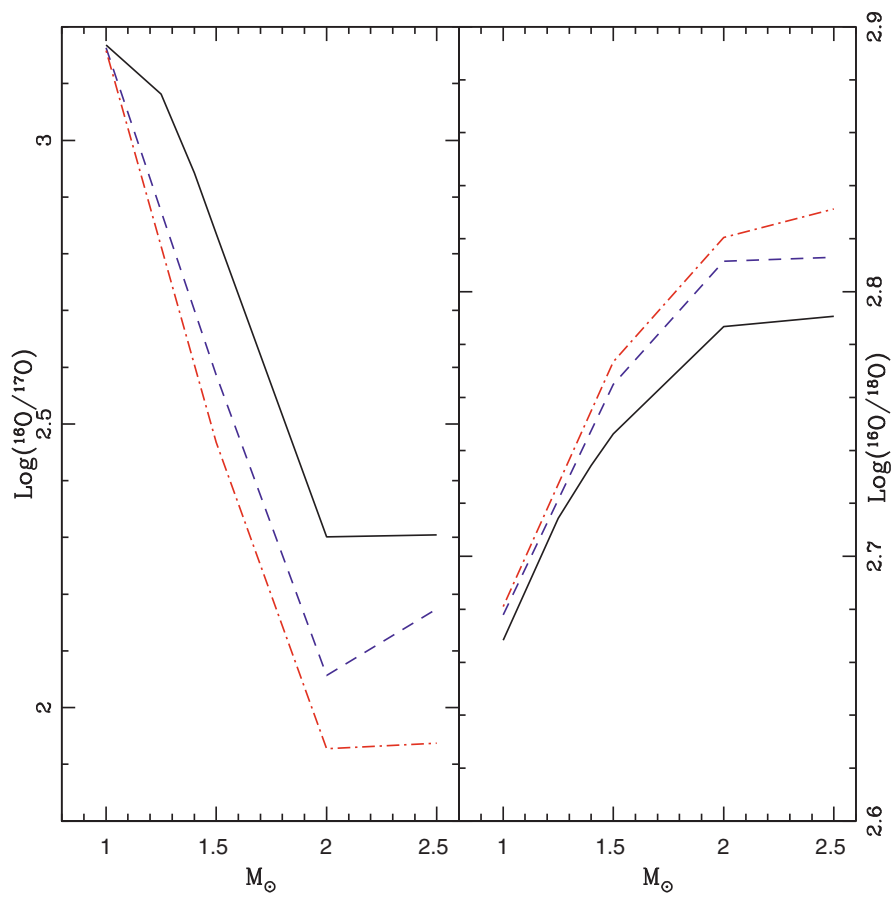

Fig. 3.31. Theoretical predictions for the surface oxygen isotopic ratios at the end of the first dredge-up as a function of the initial stellar mass for various metallicities (solar, solid line; $[\mathrm{Fe} / \mathrm{H}]=-0.56$, dashed line; $[\mathrm{Fe} / \mathrm{H}]=-0.86$, dashed-dot line). Models from Lagarde et al. (2011).

In the rotating model at the end of central helium burning, the abundance gradients are smoothed out in the radiative envelope (i.e. the region between the helium-rich region, or helium buffer, and the base of the convective envelope) compared to the standard case: ${ }^{14} \mathrm{~N}$ produced in the internal $\mathrm{H}$-burning layers diffuses outwards, while the ${ }^{12} \mathrm{C}$ and ${ }^{16} \mathrm{O}$ present in the envelope are transported inwards. As a consequence during the whole central He-burning phase, rotation-induced mixing produces a continuous (although modest) increase in ${ }^{14} \mathrm{~N}$ concomitant to a decrease of ${ }^{12} \mathrm{C}$ and ${ }^{16} \mathrm{O}$ at the stellar surface (remember that in the standard reference model presented in Sect. 3.2.3.1, the surface composition is modified by the second dredge-up only). At the same time, the products of central helium burning, namely ${ }^{12} \mathrm{C}$ and ${ }^{16} \mathrm{O}$, are transported from the core into the helium buffer (i.e. the region where helium is the dominant species, between $\sim 0.6$ and $1.4 M_{\odot}$ ) due mainly to shear turbulence. However the large mean molecular weight gradient existing at the base of the hydrogen-burning shell prevents the transport of primary $\mathrm{C}, \mathrm{N}$ and $\mathrm{O}$ from the helium buffer into the hydrogen-burning shell, and hence to the surface. At the same time, hydrogen is transported from the convective envelope inwards and is rapidly captured by ${ }^{12} \mathrm{C}$ and ${ }^{16} \mathrm{O}$ nuclei through CNO 

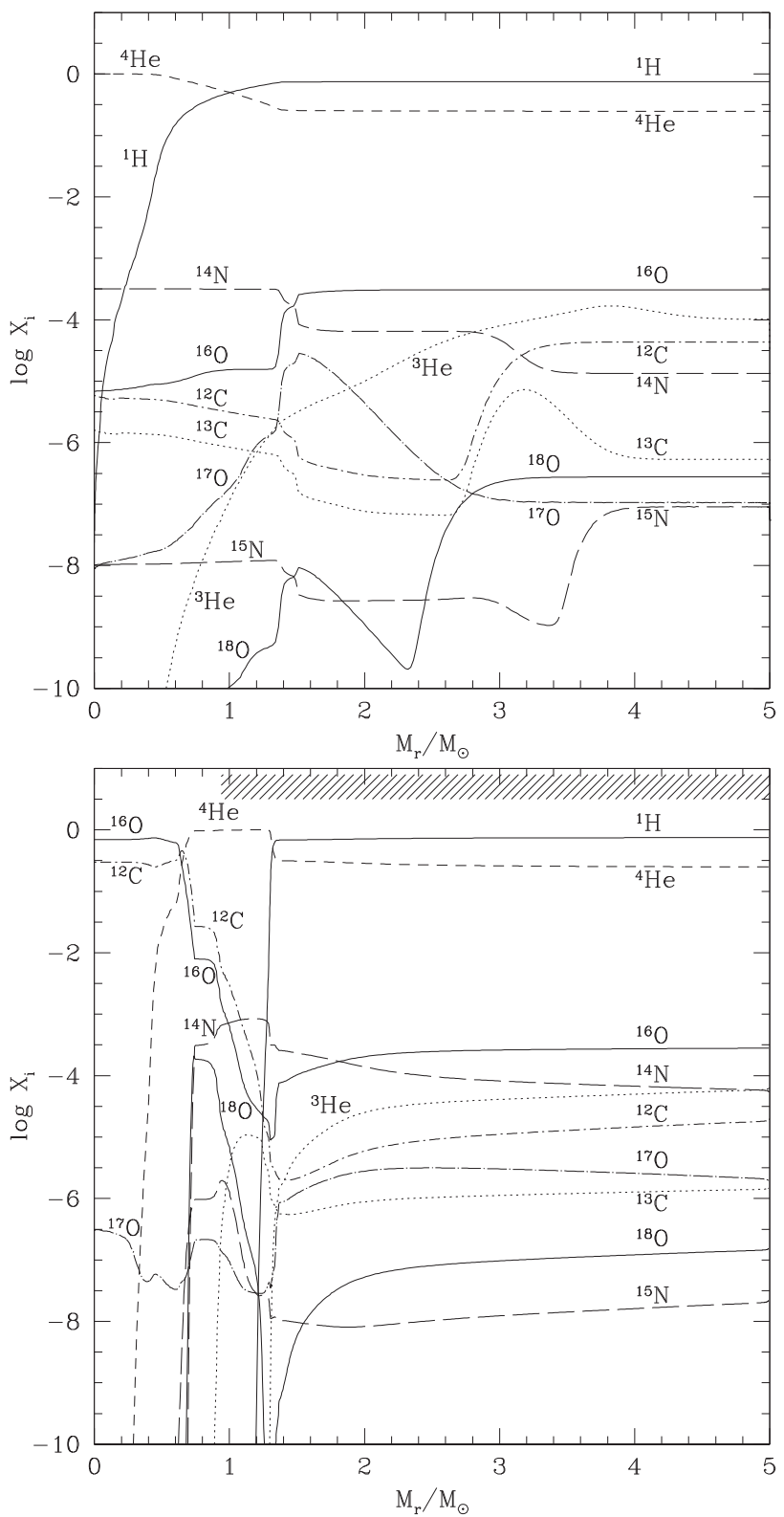

Fig. 3.32. Same as Figure 3.26 for a star with an initial mass of $5 M_{\odot}$ and $[\mathrm{Fe} / \mathrm{H}]=$ -1.56 computed with rotation (initial rotation velocity of $300 \mathrm{~km} \mathrm{~s}^{-1}$ ).

burning at high temperature, leading to the production of a peak of primary ${ }^{14} \mathrm{~N}$ at the base of the hydrogen-burning shell as can be seen in Figure 3.32 (right). 


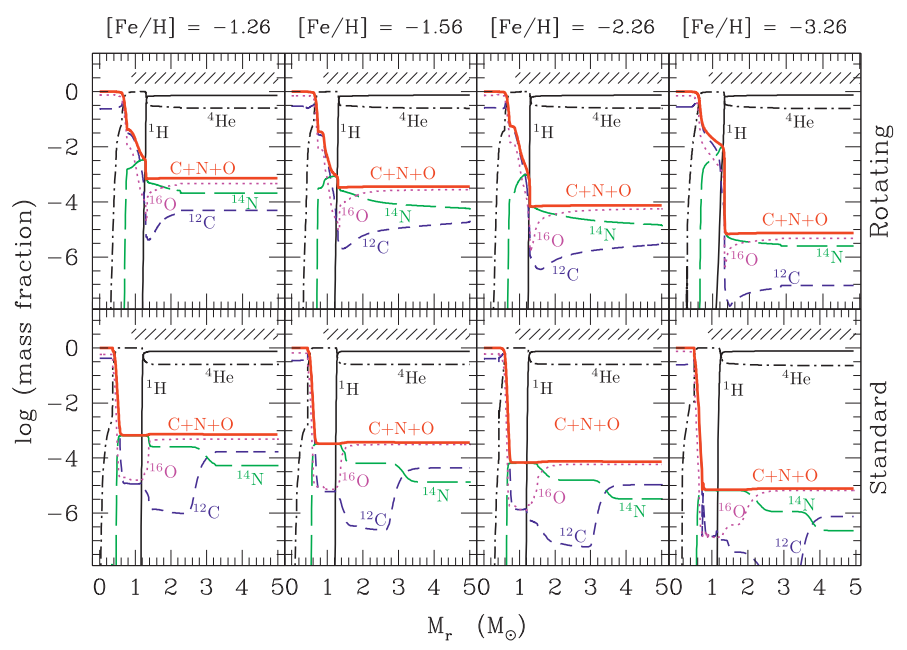

Fig. 3.33. Abundance profiles at the end of central He burning in rotating (top) and standard (bottom) $5 M_{\odot}$ models at various metallicities as indicated. The elements shown are ${ }^{1} \mathrm{H}$ (full line), ${ }^{4} \mathrm{He}$ (dotted-dashed), ${ }^{12} \mathrm{C}$ (short-dashed), ${ }^{14} \mathrm{~N}$ (long-dashed), ${ }^{16} \mathrm{O}$ (dotted) and $\mathrm{C}+\mathrm{N}+\mathrm{O}$ (thick). The hatched area on top of each panel indicates the maximum extent of the convective envelope during the second dredge-up. Figure from Decressin et al. (2009).

The resulting chemical profiles at the end of central helium burning thus differ significantly from those obtained in the standard reference case, where ${ }^{14} \mathrm{~N}$ is only produced in the hydrogen-burning shell from the ${ }^{12} \mathrm{C}$ and ${ }^{16} \mathrm{O}$ originally present in the star and, therefore, of secondary origin (Meynet \& Maeder 2002). During the second dredge-up, the convective envelope of the $5 M_{\odot}$ rotating model reaches the polluted helium buffer (hatched area in Fig. 3.32), producing a large surface abundance increase in ${ }^{4} \mathrm{He}$ and in primary ${ }^{12} \mathrm{C},{ }^{14} \mathrm{~N}$ and ${ }^{16} \mathrm{O}$. As a consequence there is an increase in the total $\mathrm{C}+\mathrm{N}+\mathrm{O}$ and overall metallicity in the envelope and thus at the stellar surface.

The impact of rotation on stellar properties and yields is known to depend strongly on metallicity (see e.g. Meynet \& Maeder 2002). The metallicity dependence for rotating $5 M_{\odot}$ stars is depicted in Figure 3.33. As discussed previously, the $\mathrm{C}+\mathrm{N}+\mathrm{O}$ profile outside the $\mathrm{CO}$ core is constant (i.e. it neither changes with time nor with depth in these layers with respect to its initial value) in the standard models, while it strongly increases in the helium buffer below the hydrogen-burning shell in the rotating models. This $\mathrm{C}+\mathrm{N}+\mathrm{O}$ step is higher in rotating stars with lower metallicity, which results in a stronger $\mathrm{C}+\mathrm{N}+\mathrm{O}$ surface enhancement after the second dredge-up in the most metal-poor stars as shown in Figure 3.34. At $[\mathrm{Fe} / \mathrm{H}]=-2.26$, the envelope (and thus the wind) of all stars more massive than $\sim 5 M_{\odot}$ undergoes a $\mathrm{C}+\mathrm{N}+\mathrm{O}$ increase of one to two orders of magnitude, while an increase of a factor of 5 is obtained at $[\mathrm{Fe} / \mathrm{H}]=-1.26$. When metallicity becomes 


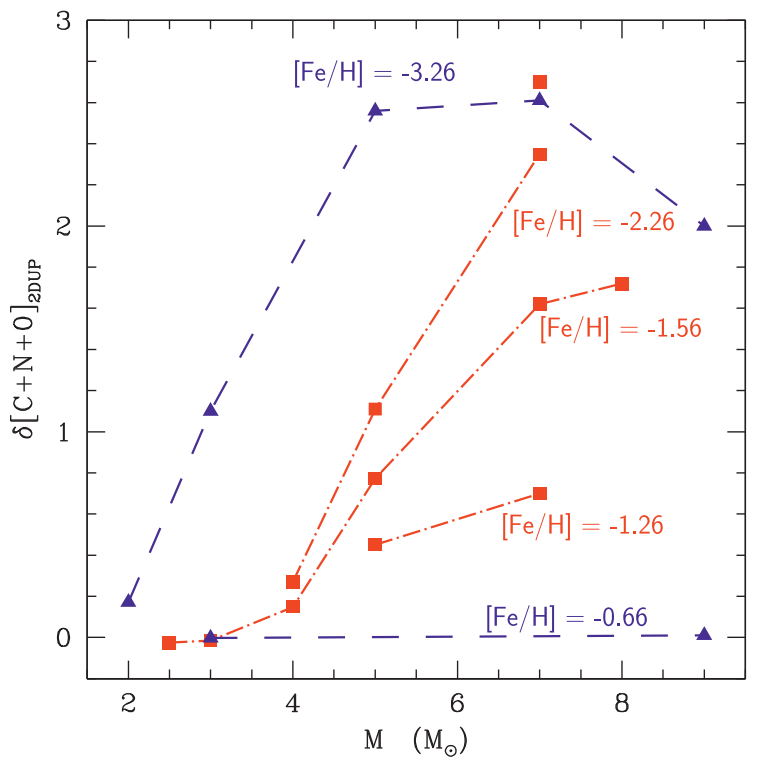

Fig. 3.34. Surface $\mathrm{C}+\mathrm{N}+\mathrm{O}$ increase index $\left(\delta[\mathrm{C}+\mathrm{N}+\mathrm{O}]_{2 \mathrm{DUP}}=[(\mathrm{C}+\mathrm{N}+\mathrm{O}) / \mathrm{Fe}]_{2 \mathrm{DUP}}-\right.$ $\left.[(\mathrm{C}+\mathrm{N}+\mathrm{O}) / \mathrm{Fe}]_{\text {ini }}\right)$ at the end of the second dredge-up for rotating stars of various initial masses and metallicities. Figure from Decressin et al. (2009).

Table 3.14. Surface abundance variations after the completion of the second dredge-up with respect to the initial composition for the models with initial value of $[\mathrm{Fe} / \mathrm{H}]=-1.56$. See Decressin et al. (2009) for more details.

\begin{tabular}{lrrrrrr}
\hline & $2.5 M_{\odot}$ & $3 M_{\odot}$ & $4 M_{\odot}$ & $5 M_{\odot}$ & $7 M_{\odot}$ & $8 M_{\odot}$ \\
\hline & 0.26 & 0.26 & 0.29 & 0.32 & 0.36 & 0.36 \\
$\mathrm{He}$ & -0.20 & -0.25 & -0.29 & -0.31 & -0.30 & -0.34 \\
$\delta[\mathrm{C} / \mathrm{Fe}]$ & 0.44 & 0.48 & 0.60 & 0.75 & 0.79 & 0.81 \\
$\delta[\mathrm{N} / \mathrm{Fe}]$ & -0.01 & -0.01 & -0.03 & -0.06 & -0.10 & -0.11 \\
$\delta[\mathrm{O} / \mathrm{Fe}]$ & 0.00 & 0.00 & 0.00 & 0.00 & 0.00 & 0.00 \\
$\delta[\mathrm{CNO} / \mathrm{Fe}]$ & 0.29 & 0.29 & 0.32 & 0.34 & 0.35 & 0.36 \\
& -1.12 & -0.86 & -0.20 & 1.44 & 2.14 & 2.16 \\
$\mathrm{He}$ & 1.23 & 1.12 & 1.13 & 1.24 & 1.19 & 1.09 \\
$\delta[\mathrm{C} / \mathrm{Fe}]$ & -0.65 & -0.36 & -0.01 & 0.18 & 1.46 & 1.55 \\
$\delta[\mathrm{N} / \mathrm{Fe}]$ & 0.00 & 0.00 & 0.15 & 0.78 & 1.62 & 1.71 \\
$\delta[\mathrm{O} / \mathrm{Fe}]$ & \multicolumn{7}{c}{ Rotating models } &
\end{tabular}

higher than $[\mathrm{Fe} / \mathrm{H}] \sim-1$, rotation-induced mixing increases the total $\mathrm{C}+\mathrm{N}+\mathrm{O}$ by less than a factor of $2-3$. The effect is null for more metal-rich stars.

Table 3.14 summarises the abundance variations after the second dredge-up in all the standard and rotating models of Decressin et al. $(2009)$ at $[\mathrm{Fe} / \mathrm{H}]=-1.56$. 
The main signature of rotational mixing at the surface of massive early-AGB stars $\left(\mathrm{M} \geq 4 M_{\odot}\right)$ is a strong increase in He-burning products, i.e. primary CNO. The total increase of $\mathrm{C}+\mathrm{N}+\mathrm{O}$ at the surface of rotating models depends mainly on the depth reached by the convective envelope during the second dredge-up; a stronger variation is thus predicted with increasing stellar mass.

\subsubsection{Yields}

As far as oxygen isotopes are concerned, low- and intermediate-mass stars at the end of their existence are net producers of ${ }^{17} \mathrm{O}$, and partially destroy ${ }^{16} \mathrm{O}$ and ${ }^{18} \mathrm{O}$. Although both the evolution of these stars and their nucleosynthesis are very well understood, the quantitative determination of reliable yields is still unreliable today. This is due to several uncertainties in the input physics of the stellar models, in particular in the mass loss rates that are crucial as far as the competition between the third dredge-up and hot bottom burning is concerned, but also in the treatment of internal transport processes of chemicals (convection, overshooting, rotation-induced mixing, thermohaline instability, etc.) during the TP-AGB phase and even during earlier evolution phases.

In view of the uncertainties we decide to show here only the results of the most recent computations published to date in the literature, that is the standard (i.e. non-rotating) models for stars of various initial masses and metallicities by Karakas (2010). Figures 3.35 and 3.36 present for each O isotope the corresponding production factor (defined as $\log _{10}\left[\langle X(i)\rangle / X_{0}(i)\right]$, where $\langle X(i)\rangle$ is the average mass fraction of species $i$ in the wind and $X_{0}(i)$ its initial mass fraction). We refer to this paper for more details and comparisons with other studies.

\subsubsection{Observational probes}

The theoretical oxygen isotope ratios can be compared with those observed in evolved stars (Harris \& Lambert 1984a,b; Harris et al. 1985b, 1987, 1985a, 1988), in interstellar grains (Nittler et al. 1994) ${ }^{8}$ and in meteorites (Huss et al. 1994). Note however that the few measurements that were made only for field giants have large observational errors that prevent a thorough comparison with model predictions, and do not allow a clear investigation of the dependence of these ratios with the stellar mass.

Figure 3.37 shows the comparison for stars with masses below $\sim 3 M_{\odot}$. The data are from Harris \& Lambert (1984a), Harris et al. (1988), and the models from Charbonnel \& Lagarde (2010) account for rotation-induced mixing and thermohaline convection (see the labels in the figure caption). In the ${ }^{16} \mathrm{O} /{ }^{17} \mathrm{O} v s$. ${ }^{16} \mathrm{O} /{ }^{18} \mathrm{O}$ plane, the post-dredge-up stellar observations lie along a roughly vertical band consistent with the theoretical predictions of first dredge-up in stars with initial oxygen isotope ratios very close to the solar values. As discussed

\footnotetext{
${ }^{8}$ Most grains form during high-mass-loss episodes of AGB stars, namely near the tip of the AGB.
} 


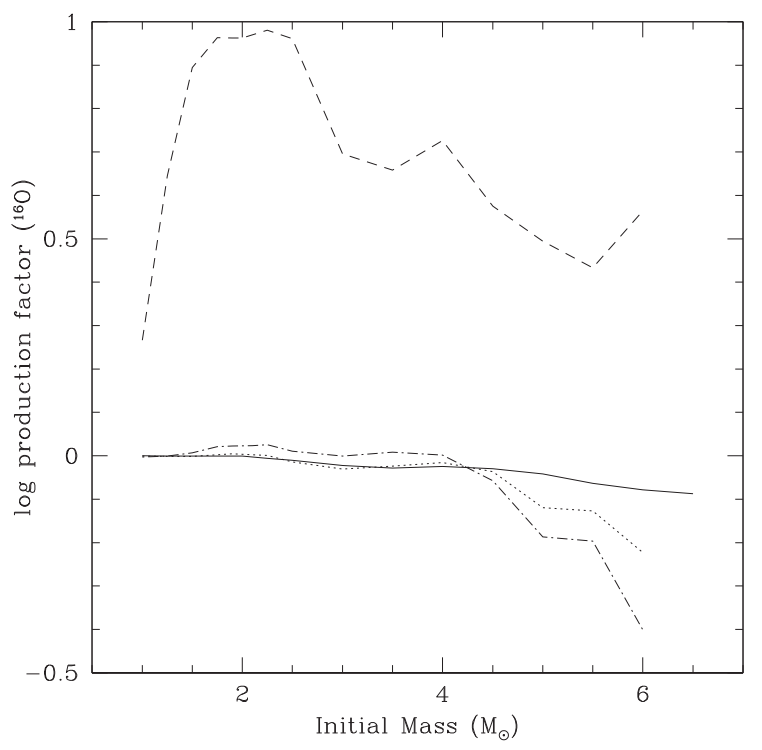

Fig. 3.35. Production factor (defined as $\left.\log _{10}[<X(i)\rangle / X_{0}(i)\right]$, where $\langle X(i)\rangle$ is the average mass fraction of species $i$ in the wind and $X_{0}(i)$ its initial mass fraction) of ${ }^{16} \mathrm{O}$ as a function of initial mass for various metallicities $(Z=0.02$, solid line; $Z=0.008$, dotted line; $Z=0.004$, dot-dashed line; $Z=0.0001$, long dashed line). The large production factor in the lowest metallicity models results from the contribution of deep third dredge-up bringing a bit of ${ }^{16} \mathrm{O}$ into an envelope with very little ${ }^{16} \mathrm{O}$ to begin with. The overall contribution of these stars to ${ }^{16} \mathrm{O}$ production should be small though, but could have important consequences when observing $\mathrm{O}$ in low metallicity planetary nebulae (see Karakas \& Lugaro 2010). Models from Karakas (2010).

in Section 3.2.3.2, thermohaline convection on the red giant branch affects only slightly the ${ }^{16} \mathrm{O} /{ }^{18} \mathrm{O}$ ratio in low-mass stars, and leaves ${ }^{16} \mathrm{O} /{ }^{17} \mathrm{O}$ unaffected. On the other hand, rotation-induced mixing lowers the ${ }^{16} \mathrm{O} /{ }^{17} \mathrm{O}$ and helps accounting for the lowest ${ }^{16} \mathrm{O} /{ }^{18} \mathrm{O}$ values of the sample stars. Given the large observational uncertainties, the predictions are reasonably consistent with the $\mathrm{O}$ isotopic ratios measured in red giant stars. Also, the data for $\mathrm{AGB}$ stars with ${ }^{12} \mathrm{C} /{ }^{13} \mathrm{C}$ near the $\mathrm{CN}$-cycle equilibrium value (not shown here) have oxygen isotope ratios consistent with theoretical predictions for intermediate-mass stars undergoing hot bottom burning.

However in the ${ }^{16} \mathrm{O} /{ }^{17} \mathrm{O}$ vs. ${ }^{16} \mathrm{O} /{ }^{18} \mathrm{O}$ plane, the oxygen isotope ratios observed in AGB stars as well as in some of the pre-solar oxide grains (Nittler et al. 1994) are in a region inaccessible to both the first dredge-up or hot bottom burning (see e.g. Fig. 7 in Boothroyd \& Sackmann 1999). Cool carbon stars (the so-called J-type stars) show rather low ${ }^{16} \mathrm{O} /{ }^{17} \mathrm{O}$ isotopic ratios while only upper limits have been found for the ${ }^{16} \mathrm{O} /{ }^{18} \mathrm{O}$ ratio. Most of the other AGB stars present ${ }^{16} \mathrm{O} /{ }^{17} \mathrm{O}$ 

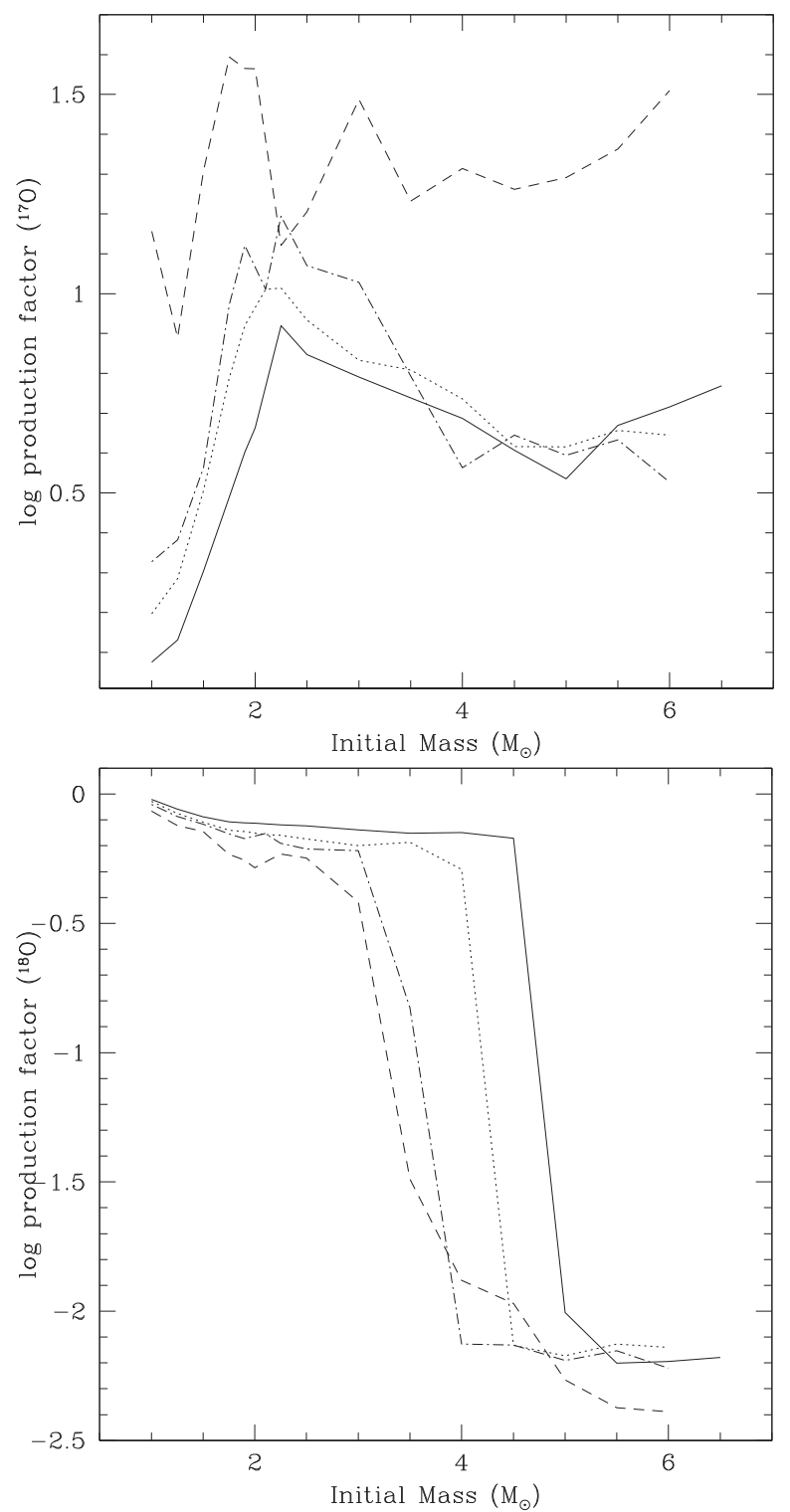

Fig. 3.36. Same as Figure 3.35 for ${ }^{17} \mathrm{O}$ and ${ }^{18} \mathrm{O}$.

ratios between 300 and 4000 and ${ }^{16} \mathrm{O} /{ }^{18} \mathrm{O}$ ratios between 500 and 5000. Stars with rather high ${ }^{16} \mathrm{O} /{ }^{17} \mathrm{O}$ ratios are difficult to explain with intermediate-mass AGB models (see e.g. Boothroyd et al. 1995). Let us however emphasise that part of the discrepancy may be due to the important uncertainties that remain concerning the nuclear cross sections of proton captures on ${ }^{17} \mathrm{O}$. 


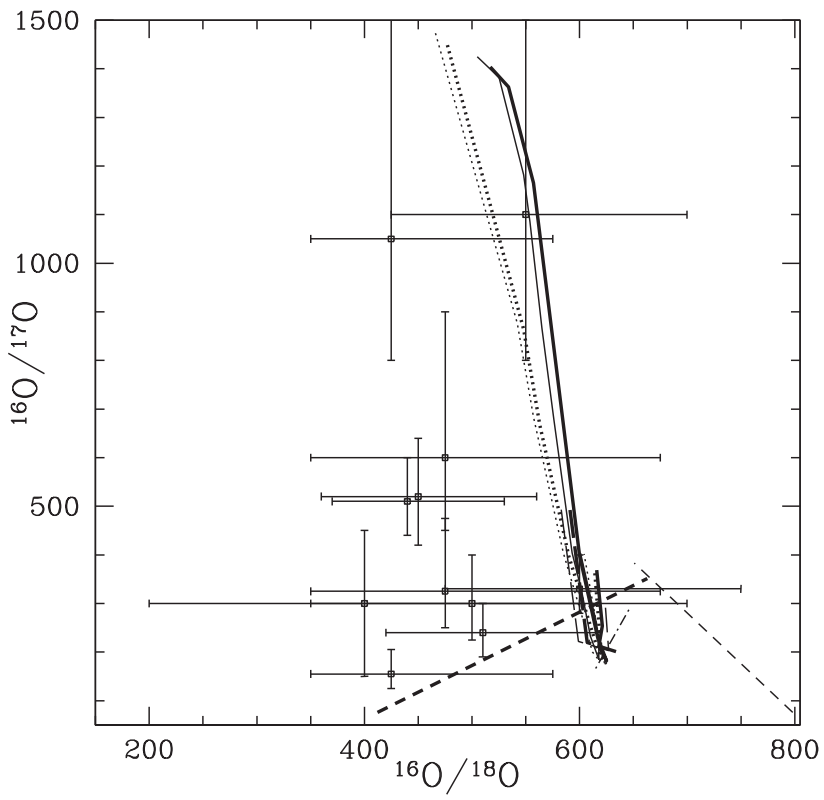

Fig. 3.37. ${ }^{16} \mathrm{O} /{ }^{17} \mathrm{O}$ vs. ${ }^{16} \mathrm{O} /{ }^{18} \mathrm{O}$ observed by Harris \& Lambert (1984a); Harris et al. (1988) for red giant stars in the 1-3 $M_{\odot}$ range. The lines are predictions for solar metallicity models by Charbonnel \& Lagarde (2010). Theoretical predictions are shown at the tip of the RGB and after completion of the second dredge-up (thin and thick continuous lines respectively). Standard models (neither thermohaline nor rotation-induced mixing) are shown as dotted lines, models with thermohaline mixing only (no rotation) as solid lines and models with thermohaline and rotation-induced mixing for different initial rotation velocities as long-dashed, dot-dashed and dashed lines. Figure from Charbonnel \& Lagarde (2010).

On that question grain data are more precise than stellar data. Indeed, while many of the grain data are consistent with dredge-up in stars of near-solar metallicity, roughly a dozen grains show ${ }^{18} \mathrm{O}$ depletion by factors $\geq 3$, and several of these have ${ }^{17} \mathrm{O}$ abundances a factor of 2 lower than would be expected by AGB stars undergoing hot bottom burning. In view of the relatively large ${ }^{12} \mathrm{C} /{ }^{13} \mathrm{C}$ ratios observed in most of these AGB stars, the non-canonical mixing that could lead to the observed patterns is suspected to occur on the RGB rather than on the AGB (see Sect. 2.2.3.2).

Finally, another very interesting way to constrain stellar evolution models of AGB stars is to compare the predicted surface isotopic ratios with those determined in primitive meteorites (mainly through grains included inside carbon rich chondrites). For example, measurements of the $\mathrm{O}$ isotopic ratios have been made on a $\sim 3 \mu \mathrm{m} \mathrm{Al}_{2} \mathrm{O}_{3}$ grain of the Bishunpur LL3.1 chondrite by Huss et al. (1994). The derived ratios, ${ }^{16} \mathrm{O} /{ }^{17} \mathrm{O}=385 \pm 5$ and ${ }^{16} \mathrm{O} /{ }^{18} \mathrm{O}=853 \pm 30$, are in perfect agreement with ratios corresponding to a 3-4 $M_{\odot}$ AGB star of nearly solar 
metallicity. This is of course in favour of the presence, when the Solar System was formed, of one or a few AGB stars in its surroundings. Such grains are indeed well known to be formed in the cool atmosphere of evolved AGB stars (i.e. when mass loss rates become rather high).

\subsubsection{Binary stars}

Many massive stars belong to multiple systems (Zinnecker 2008), a subset of which will go through mass transfer episodes. In such cases, the evolution of the massive star will be modified with respect to that it would have had in isolation. Also, before any mass transfer event actually occurs, tidal forces in close binary systems probably trigger instabilities inside the star that modify its structure.

A lot of work remains to be done in this area of research, and also the range of parameters to be explored is much more extended than in a single star evolutionary scenario. In addition to mass, metallicity and rotation which are, among others, very important properties of single stars, mass ratios and orbital periods are needed to specify the evolution of a binary system.

At present chemical evolution models of galaxies account for binary scenarios only through Type Ia supernovae. Therefore, it is difficult to assess the importance of close binary evolution in the synthesis of oxygen. Langer (2003) provides an interesting discussion of this question. He first makes a very general comment on the importance of a given star population in the synthesis of a peculiar element. If that population represents a fraction $f$ of the whole stellar population, in order to contribute in a significant way to the synthesis of that element, the respective yield in that population needs to be larger by a factor $1 / f$ than the corresponding single star yield. Applied to binary systems, if we take $f=0.5$ (clearly an overestimate since not all binary systems have components that will undergo a mass transfer episode), then the yield would be enhanced by about a factor of 2 (here an underestimate) with respect to the single star scenario for binary evolution to have a significant impact.

Regarding ${ }^{16} \mathrm{O}$, although in some peculiar situations the enhancement factor can indeed be greater than a factor of 2 , it seems reasonable to think that close binary evolution will not significantly distort the single star scenario as far as chemical evolution is concerned (Langer 2003). For the other two isotopes, the situation may be different, but remains difficult to be assessed in a quantitative way.

\subsection{Final comments}

Let us end this chapter emphasising two important aspects.

1. Massive stars are the sources of ${ }^{16} \mathrm{O}$ in the Universe. The reasons supporting this assertion are the following.

- An analysis of the surface abundances of very metal-poor halo stars indicates a high oxygen to iron ratio. According to chemical evolution 
models (see Chapter 4), this reflects the fact that these stars are formed from material which has been enriched by oxygen-rich ejecta from massive stars.

- Observations of the surface abundances of naked He-burning stellar cores, namely WC and WO stars, confirm that nuclear burning reactions produce large amounts of oxygen.

- Since He-burning also occurs in low and intermediate mass stars, why do these types of stars hardly contribute to ${ }^{16} \mathrm{O}$ synthesis? The reason is that in these stars most, if not all, of the material processed by $\mathrm{He}$ burning will remain locked in the remnant (a white dwarf), and thus will not be ejected into the interstellar medium at the end of the star lifetime. For massive stars, the CO-core masses are sufficiently large for a significant fraction to be ejected by the stellar winds (in the case of the WC-WO stars) or at the time of the supernova explosion.

2. In order to constrain the predictions of galaxy chemical evolution models, which aim at reproducing observed abundance patterns in the Universe (see Chapter 4), the abundances observed at the surface of stars are sometimes used. This is of course legitimate provided the surface abundances reflect those of the ISM at the birth of the star, i.e. if the surface abundances have not changed during the stellar lifetime. The causes for changing the surface abundances can be mass loss, mass accretion from a close companion and internal mixing processes induced by, for instance, rotation or convection. The most interesting targets for constraining the chemical evolution models are single, low-mass main-sequence stars (which are slow rotators). Even in that case, various diffusion processes may change the surface abundances, but the situation is much more reliable than looking at the surface abundances of more evolved stars. At least, any other choice must be carefully considered and critically discussed with respect to any process which may have changed the surface abundances. This is the case, for example, when using planetary nebulae as oxygen abundance indicators in galaxies, as discussed in Chapter 2. 
